\title{
Self-Assembly of Concentric Hexagons and Hierarchical Self-Assembly of Supramolecular Metal-Organic Nanoribbons at Solid/Liquid Interface
}

Ming Wang, Kun Wang, Chao Wang, Mingjun Huang, Xin-Qi Hao, Ming-Zhan Shen, Guo-Qing Shi, Zhe Zhang, Bo Song, Alejandro Cisneros, Mao-Ping Song, Bingqian $\mathrm{Xu}$ and Xiaopeng Li

\section{Table of Contents}

1. Experimental section...................................................

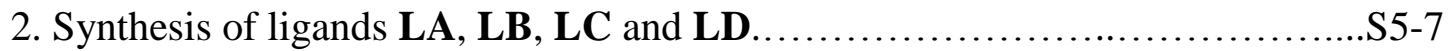

3. Synthesis of the ligands and complexes..............................S8-34

4. ESI mass spectra data of $\left[\mathbf{Z n}_{12} \mathbf{L} \mathbf{A}_{\mathbf{6}}\right], \quad\left[\mathbf{Z n}_{12} \mathbf{L} \mathbf{B}_{\mathbf{6}}\right], \quad\left[\mathbf{Z n}_{12} \mathbf{L} \mathbf{C}_{6}\right]$ and

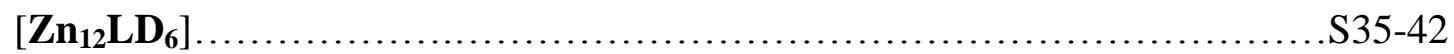

5. Energy-minimized structure from molecular modeling, ESI-MS and TWIM-MS

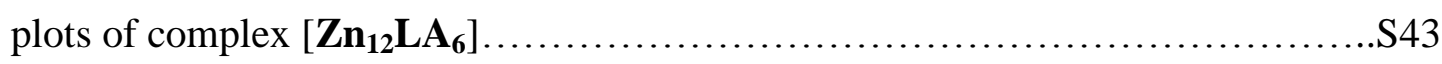

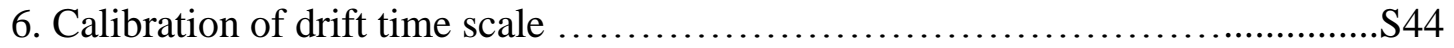

7. Molecular modeling results.......................................S45-47

8. ${ }^{1} \mathrm{H}$ NMR, ${ }^{13} \mathrm{C}$ NMR, 2D COSY NMR, 2D NOESY NMR and MALDI-TOF.S48-63

9. ESI-MS of multiple macrocycles assembled by ditopic tpy ligand 6 with $\mathrm{Zn}^{2+} \ldots \mathrm{S} 64$

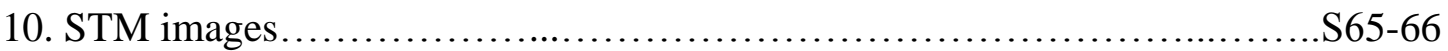

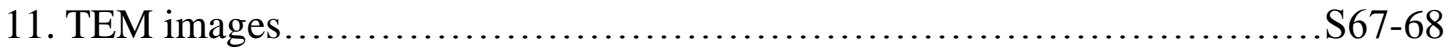

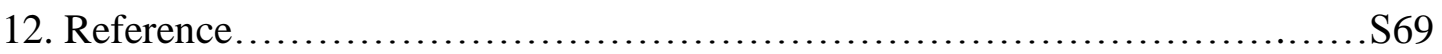




\section{Experimental section}

General Procedures. All reagents were purchased from Sigma-Aldrich, Matrix Scientific, Alfa Aesar and used without further purification. Column chromatography was conducted using basic $\mathrm{Al}_{2} \mathrm{O}_{3}$ (Brockman I, activity, $58 \AA$ ) or $\mathrm{SiO}_{2}$ (VWR, 40-60 $\mu \mathrm{m}, 60 \AA$ ) and the separated products were visualized by UV light. NMR spectra data were recorded on a $400-\mathrm{MHz}$ and $500 \mathrm{MHz}$ Bruker Avance NMR spectrometer in $\mathrm{CDCl}_{3}, \mathrm{CD}_{3} \mathrm{CN}$ or DMSO- $d 6$ with TMS as reference. ESI-MS and TWIM-MS were recorded with a Waters Synapt G2 tandem mass spectrometer, using solutions of 0.01 mg sample in $1 \mathrm{~mL}$ of $\mathrm{CHCl}_{3} / \mathrm{CH}_{3} \mathrm{OH}(1: 3, \mathrm{v} / \mathrm{v})$ for ligands or $0.5 \mathrm{mg}$ sample in $1 \mathrm{~mL}$ of $\mathrm{MeCN} / \mathrm{MeOH}(3: 1, \mathrm{v} / \mathrm{v})$ for complexes. MALDI-TOF was carried out on Bruker AutoFlex TOF/TOF mass spectrometer.

TWIM-MS. The TWIM-MS experiments were performed under the following conditions: ESI capillary voltage, $3 \mathrm{kV}$; sample cone voltage, $30 \mathrm{~V}$; extraction cone voltage, $3.5 \mathrm{~V}$; source temperature $100{ }^{\circ} \mathrm{C}$; desolvation temperature, $100{ }^{\circ} \mathrm{C}$; cone gas flow, $10 \mathrm{~L} / \mathrm{h}$; desolvation gas flow, $700 \mathrm{~L} / \mathrm{h}\left(\mathrm{N}_{2}\right)$; source gas control, $0 \mathrm{~mL} / \mathrm{min}$; trap gas control, $2 \mathrm{~mL} / \mathrm{min}$; helium cell gas control, $100 \mathrm{~mL} / \mathrm{min}$; ion mobility (IM) cell gas control, $30 \mathrm{~mL} / \mathrm{min}$; sample flow rate, $5 \mu \mathrm{L} / \mathrm{min}$; IM traveling wave height, $25 \mathrm{~V}$; and IM traveling wave velocity, $1000 \mathrm{~m} / \mathrm{s}$. Q was set in rf-only mode to transmit all ions produced by ESI into the triwave region for the acquisition of TWIM-MS data.

Collision cross-section calibration. The calibration procedure of Scrivens et al ${ }^{1}$ was used to convert the drift time scale of the TWIM-MS experiments to a collision 
cross-section (CCS) scale. The calibration curve was constructed by plotting the corrected CCSs of the molecular ions of myoglobin ${ }^{2}$ against the corrected drift times of the corresponding molecular ions measured in TWIM-MS experiments at the same traveling wave velocity, traveling wave height and ion mobility gas flow settings viz., $1000 \mathrm{~m} / \mathrm{s}, 25 \mathrm{~V}$, and $30 \mathrm{~mL} / \mathrm{min}$, respectively.

Molecular modeling. Energy minimization of the macrocycles was conducted with Materials Studio version 4.2, using the Anneal and Geometry Optimization tasks in the Forcite module (Accelrys Software, Inc.). All counterions are omitted. An initially energy-minimized structure was subjected to 70 - 100 annealing cycles with initial and mid-cycle temperatures of 300 and $1500 \mathrm{~K}$, respectively, twenty heating ramps per cycle, one thousand dynamic steps per ramp, and one femtosecond per dynamic step. A constant volume/constant energy (NVE) ensemble was used and the geometry was optimized after each cycle. Geometry optimization used a universal force field with atom-based summation and cubic spline truncation for both the electrostatic and Van der Waals parameters. 70 - 100 energy-minimized structures were selected for the calculation of theoretical collision cross-sections using MOBCAL programs.

TEM: The sample was dissolved in $\mathrm{CH}_{3} \mathrm{CN}$ at a concentration of $10^{-6} \mathrm{M}$. The solutions were drop cast on to a carbon-coated $\mathrm{Cu}$ grid and extra solution was absorbed by filter paper to avoid aggregation. The TEM images of the drop cast samples were taken with a JEOL 2010 transmission electron microscope.

STM: The sample was dissolved in DMF at a concentration of $5.0 \mathrm{mg} / \mathrm{ml}$. 
Solution (5ul) was dropped on HOPG surface. After 30 seconds, surface was washed slightly with water for three times and totally dried in R.T. in air. The STM images were taken with a PicoPlus SPM system with a PicoScan 3000 Controller. The obtained STM images were processed by WSxM software. ${ }^{3}$ 
2. Synthesis of ligands LA, LB, LC and LD

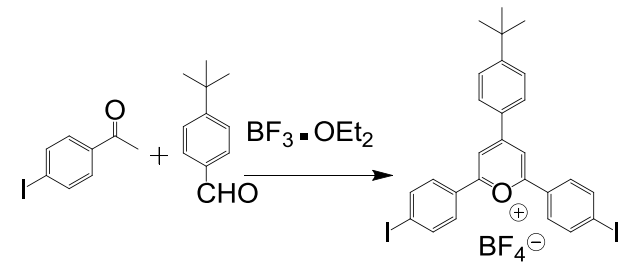

1

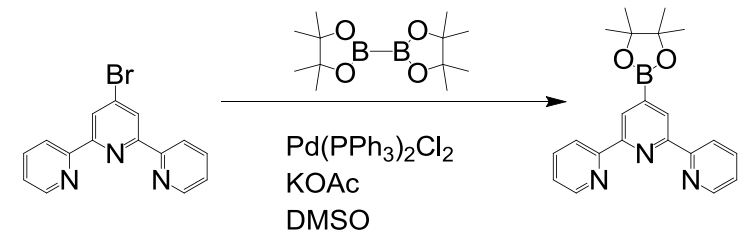

2

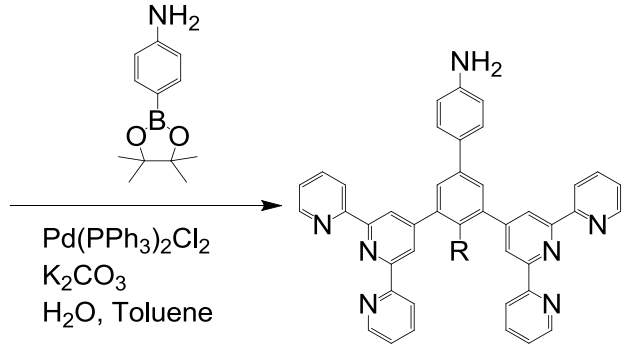

$7 \mathrm{R}=\mathrm{H}$

$5 \mathrm{R}=\mathrm{H}$

$6 \mathrm{R}=\mathrm{OC}_{6} \mathrm{H}_{13}$

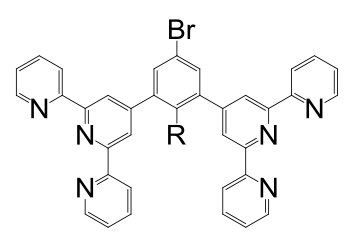

$\mathrm{H}_{2} \mathrm{O}$, Toluene $8 \mathrm{R}=\mathrm{OC}_{6} \mathrm{H}_{13}$

$4 \mathrm{R}=\mathrm{OC}_{6} \mathrm{H}_{13}$
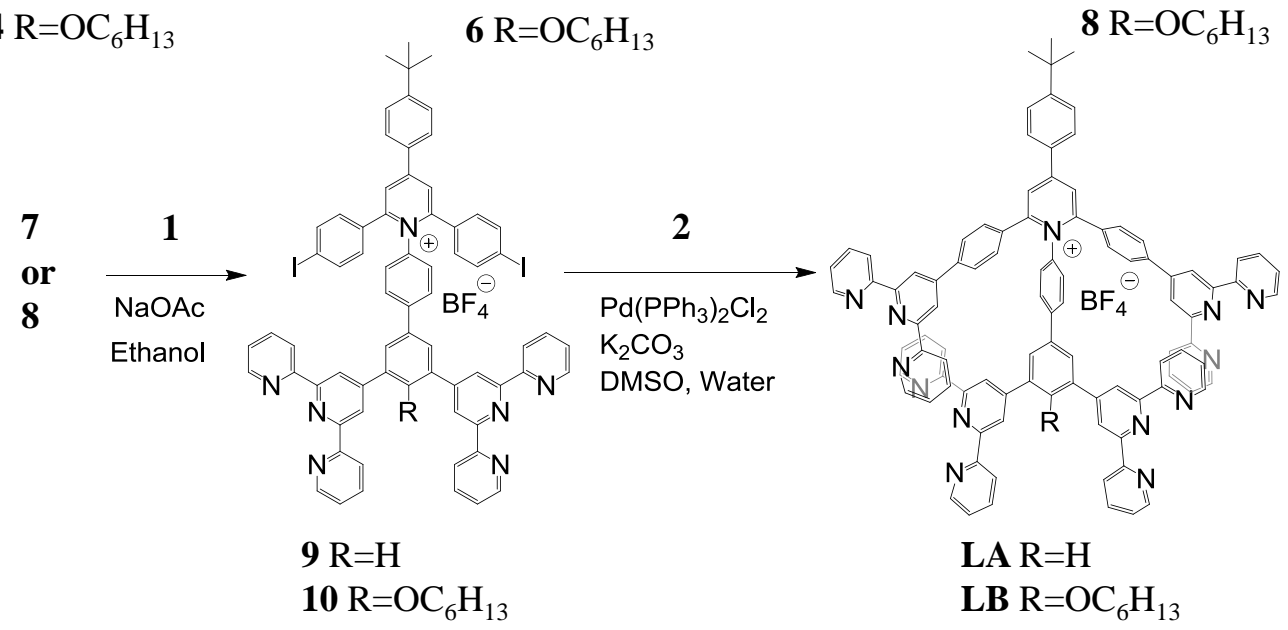

$10 \mathrm{R}=\mathrm{OC}_{6} \mathrm{H}_{13}$

LA R $=\mathrm{H}$

LB $\mathrm{R}=\mathrm{OC}_{6} \mathrm{H}_{13}$

Scheme 1. Synthesis of ligands LA and LB. 


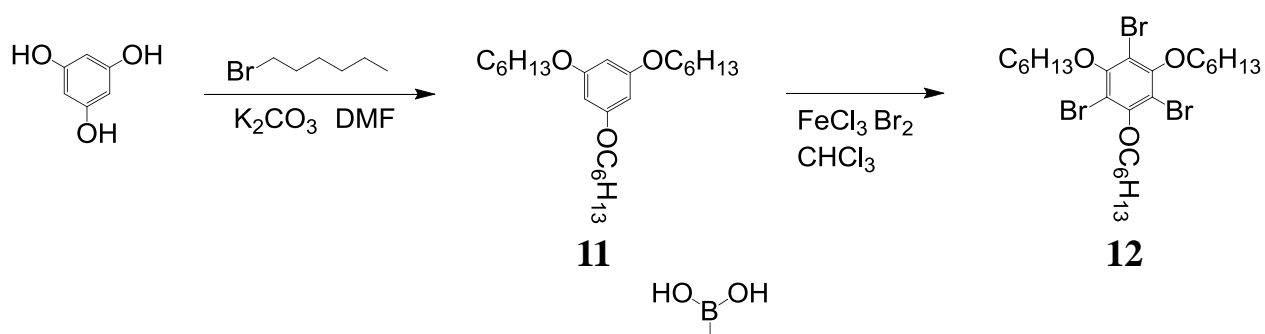

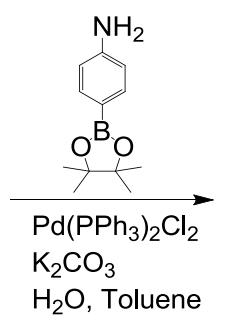

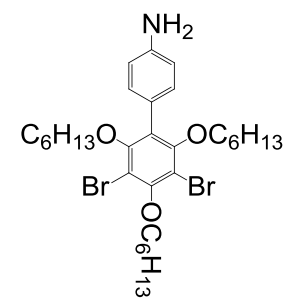

13

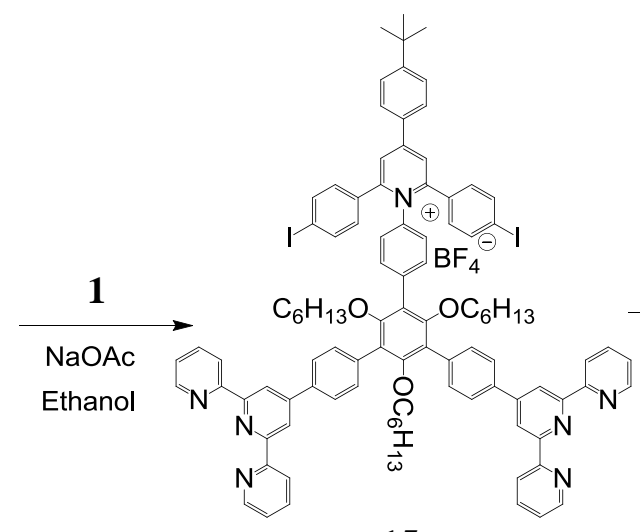

15

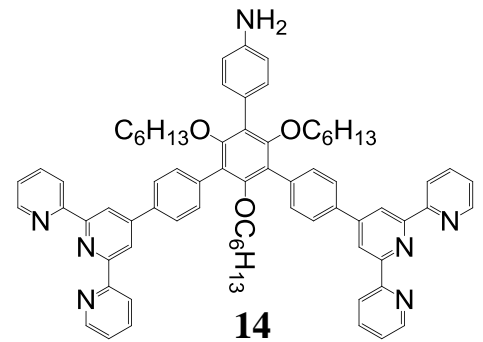

14
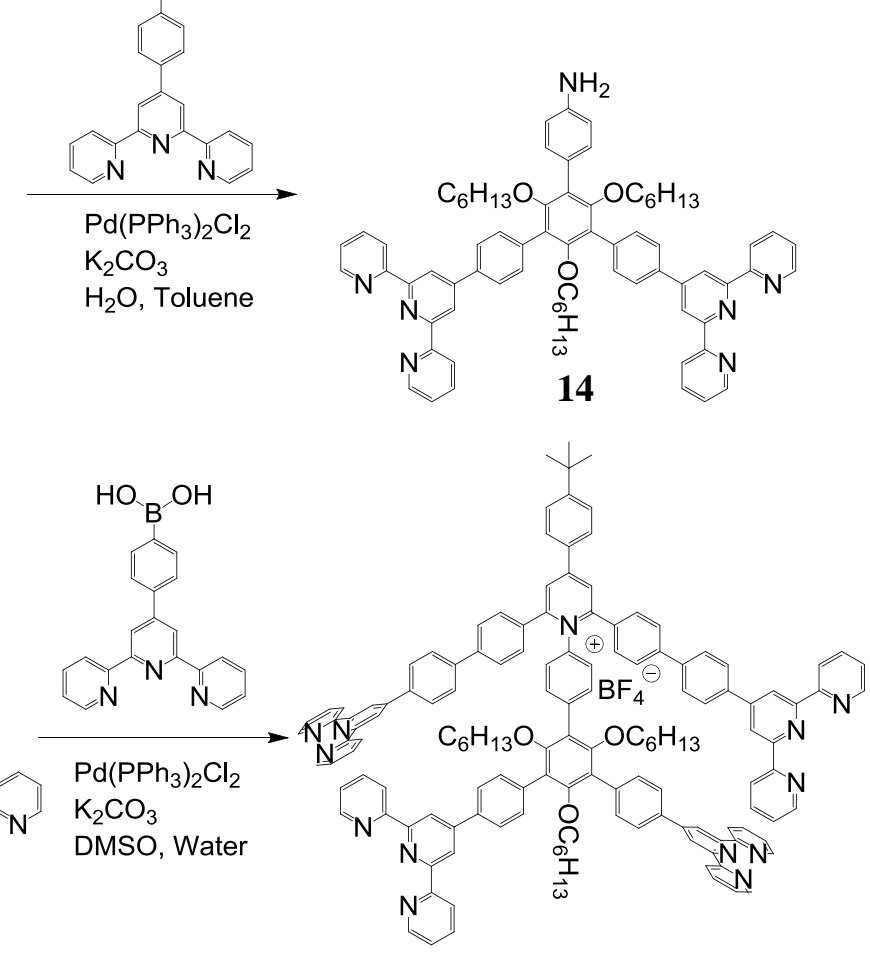

LC

Scheme 2. Synthesis of ligand LC. 

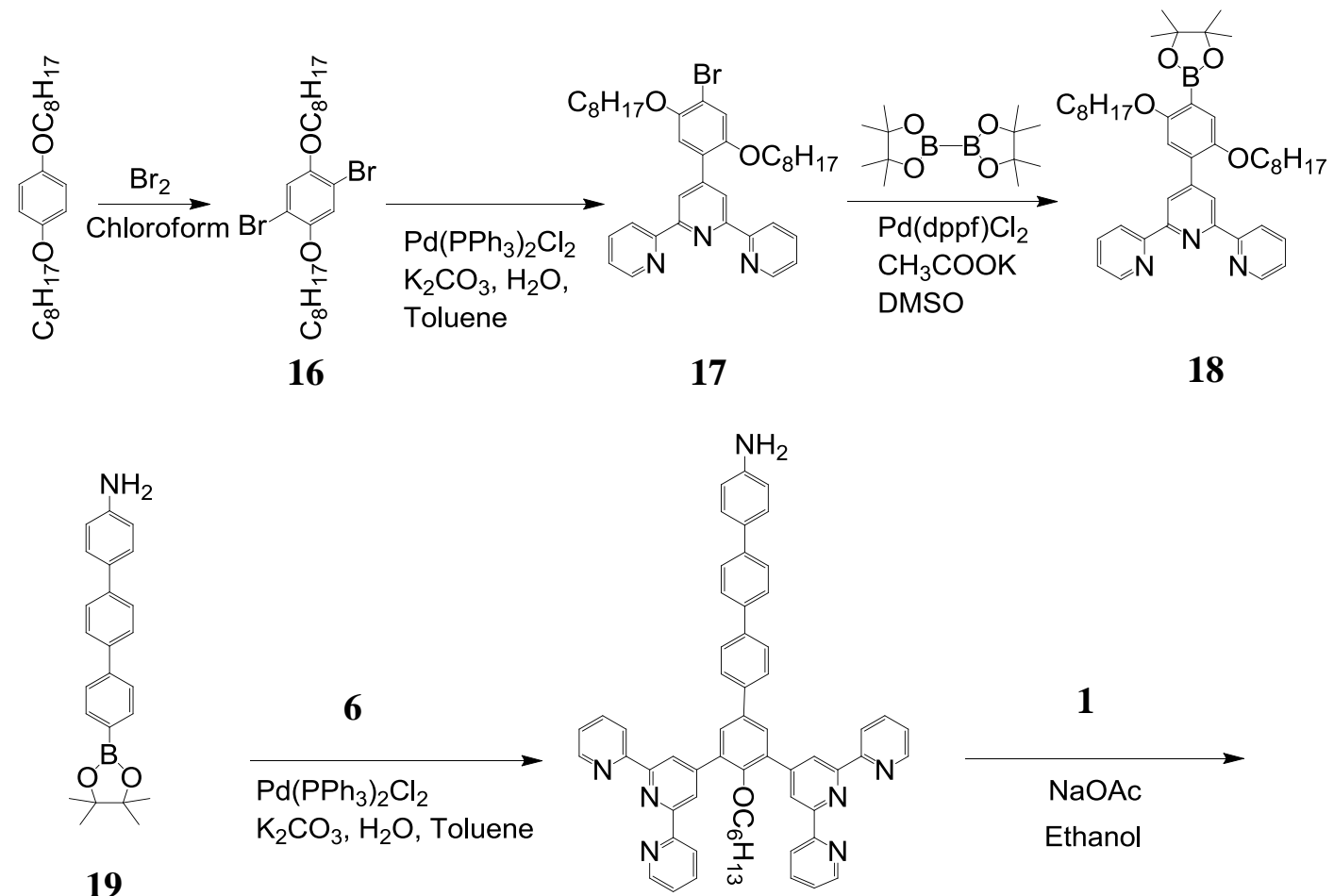

19

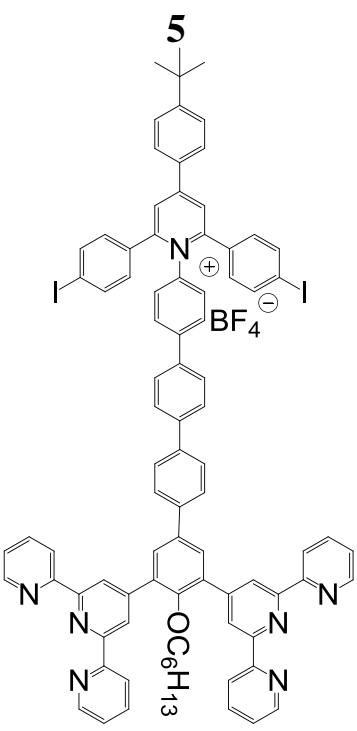

21
20

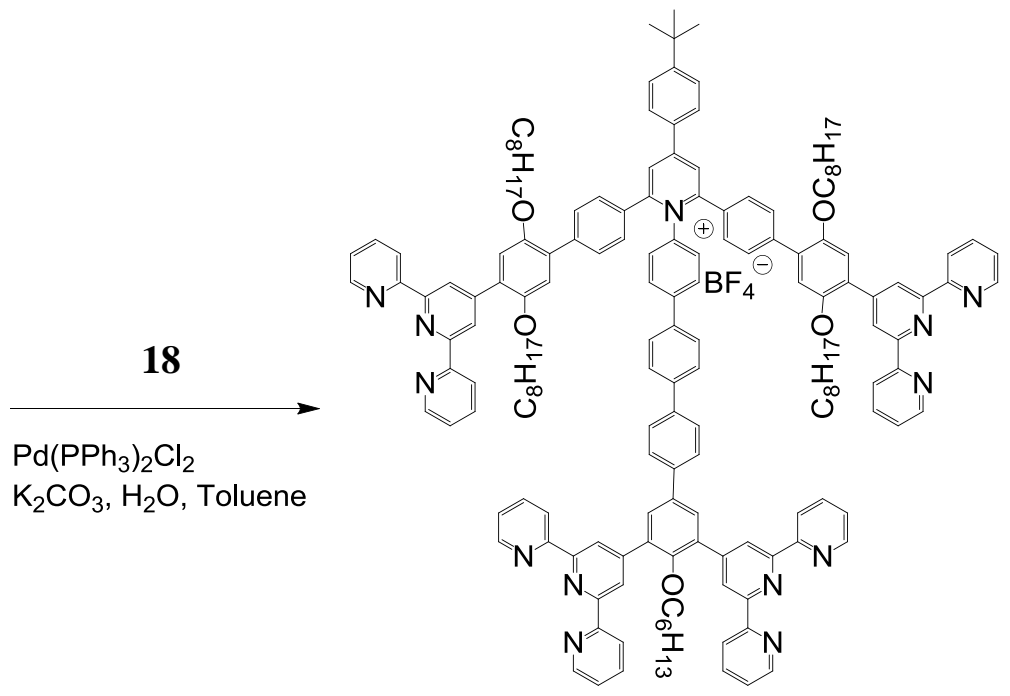

LD

Scheme 3. Synthesis of ligand LD. 


\section{Synthesis of the ligands and complexes}

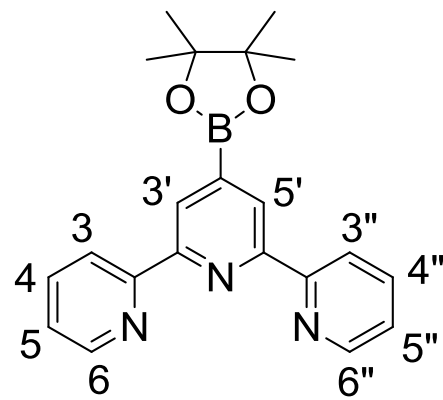

Compound 2. The mixture of 4-Brom-terpyridine ${ }^{4}$ (3.12 g, $\left.10 \mathrm{mmol}\right)$, Bis-

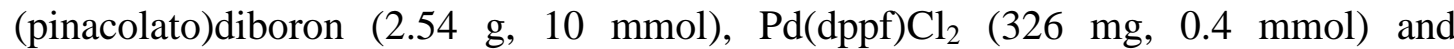
potassium acetate $(2.9 \mathrm{~g}, 30 \mathrm{mmol})$ were added $35 \mathrm{~mL}$ anhydrous DMSO. The solution was heated at $85{ }^{\circ} \mathrm{C}$ for $8 \mathrm{~h}$. The crude product was directly used in the following step. ${ }^{1} \mathrm{H}$ NMR $\left(400 \mathrm{MHz}, \mathrm{CDCl}_{3}\right) \delta 8.83\left(\mathrm{~s}, 2 \mathrm{H}, \mathrm{tpy}-H^{3^{\prime} 5^{\prime}}\right), 8.74(\mathrm{ddd}, J=$ 4.8, $\left.1.8,0.9 \mathrm{~Hz}, 2 \mathrm{H}, \mathrm{tpy}-H^{6,6^{\prime \prime}}\right), 8.62\left(\mathrm{dt}, J=8.0,1.1 \mathrm{~Hz}, 2 \mathrm{H}, \mathrm{tpy}-H^{3,3 "}\right), 7.86(\mathrm{ddd}, J=$ 8.0, 7.5, $\left.1.8 \mathrm{~Hz}, 2 \mathrm{H}, \mathrm{tpy}-H^{4,4 "}\right), 7.34\left(\mathrm{ddd}, J=7.5,4.8,1.2 \mathrm{~Hz}, 2 \mathrm{H}, \mathrm{tpy}-H^{5,5^{\prime \prime}}\right), 1.40$ (s, $\left.6 \mathrm{H}, H^{\mathrm{A}}\right) .{ }^{13} \mathrm{C} \mathrm{NMR}\left(100 \mathrm{MHz}, \mathrm{CDCl}_{3}\right) \delta 156.49,154.76,149.15,136.76,126.27$, 123.60, 121.22, 84.48, 24.95. ESI-MS (m/z): Calcd. for $\left[\mathrm{C}_{21} \mathrm{H}_{22} \mathrm{BN}_{3} \mathrm{O}_{2}+\mathrm{H}\right]^{+}: 360.2$. Found: 360.2 .

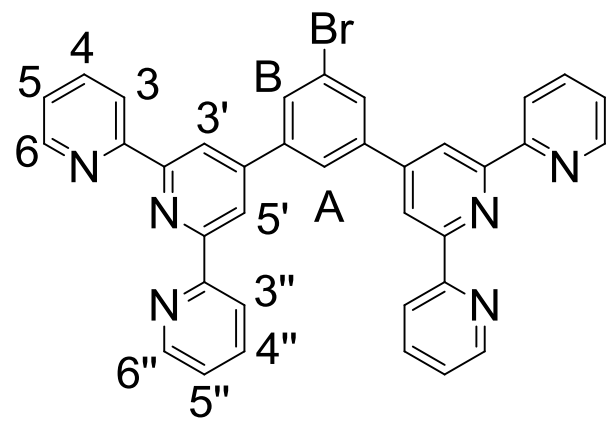

Compound 5. To a solution of $\mathrm{NaOH}$ powder (4.8 g, $120 \mathrm{mmol})$ in $\mathrm{EtOH}(100 \mathrm{ml})$, 5-bromoisophthalaldehyde ${ }^{5}(2.1 \mathrm{~g}, 10 \mathrm{mmol})$ and 2-acetylpyridine $(3.0 \mathrm{~g}, 25 \mathrm{mmol})$ was added. After stirring at room temperature for $24 \mathrm{~h}$, aqueous $\mathrm{NH}_{3} \bullet \mathrm{H}_{2} \mathrm{O}(35 \mathrm{~mL})$ 
was added and the mixture was refluxed for $40 \mathrm{~h}$. After cooling to room temperature, the precipitate was filtered. The crude was purified by column chromatography on silica gel with chloroform: ethanol (100:2) as eluent to afford the product as a white solid (2.8 g, 45\%). ${ }^{1} \mathrm{H}$ NMR (400 MHz, $\left.\mathrm{CDCl}_{3}\right) \delta 8.80$ (s, 4H, tpy- $\left.H^{3^{\prime}, 5^{\prime}}\right), 8.77(\mathrm{ddd}, J$ $=4.7,1.7,0.8 \mathrm{~Hz}, 4 \mathrm{H}$, tpy- $\left.H^{6,6 "}\right), 8.73\left(\mathrm{~d}, J=8.0 \mathrm{~Hz}, 4 \mathrm{H}, \mathrm{tpy}-H^{3,3 "}\right), 8.27(\mathrm{t}, J=1.6$ $\left.\mathrm{Hz}, 1 \mathrm{H}, \mathrm{Ph}-H^{\mathrm{A}}\right), 8.13\left(\mathrm{~d}, J=1.6 \mathrm{~Hz}, 2 \mathrm{H}, \mathrm{Ph}-H^{\mathrm{B}}\right), 7.93(\mathrm{td}, J=7.7,1.8 \mathrm{~Hz}, 4 \mathrm{H}$, tpy- $\left.H^{4,4 "}\right), 7.40\left(\mathrm{ddd}, J=7.4,4.8,1.2 \mathrm{~Hz}, 4 \mathrm{H}, \mathrm{tpy}-H^{5,5 "}\right) .{ }^{13} \mathrm{C} \mathrm{NMR}\left(100 \mathrm{MHz}, \mathrm{CDCl}_{3}\right)$ $\delta 156.17,155.96,149.15,148.69,141.45,136.89,130.75,125.10,123.98,123.73$, 121.44, 119.06. ESI-MS (m/z): Calcd. for $\left[\mathrm{C}_{36} \mathrm{H}_{23} \mathrm{BrN}_{6}+\mathrm{H}\right]^{+}$: 619.1. Found: 619.1 .

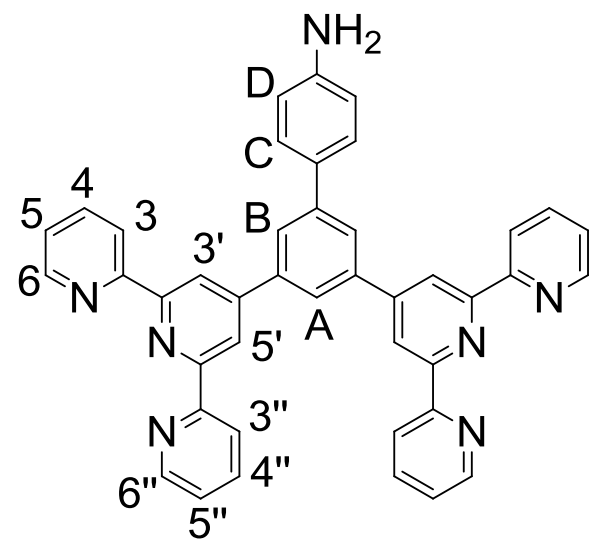

Compound 7. To a flask containing a degassed solution of compound 5 (620 mg, 1.0 mmol), 4-(4,4,5,5-tetramethyl-1,3,2-dioxaborolan-2-yl)aniline (330 mg, $1.5 \mathrm{mmol})$ in toluene $(40 \mathrm{~mL})$, an aqueous solution of $2 \mathrm{M} \mathrm{K}_{2} \mathrm{CO}_{3}(15 \mathrm{~mL})$ was added. $\mathrm{Pd}\left(\mathrm{PPh}_{3}\right)_{2} \mathrm{Cl}_{2}(70 \mathrm{mg}, 0.1 \mathrm{mmol})$ was then added under $\mathrm{N}_{2}$. The mixture was stirred at $90{ }^{\circ} \mathrm{C}$ for $24 \mathrm{~h}$ and then cooled to room temperature. The aqueous phase was extracted with $\mathrm{CH}_{2} \mathrm{Cl}_{2}$. The combined organic phase was washed with brine and dried with anhydrous $\mathrm{Na}_{2} \mathrm{SO}_{4}$. After removal of volatile under vacuum, the crude was purified by column chromatography on silica gel with chloroform: ethanol $(100: 1)$ as eluent to 
afford the product as a white solid (505 mg, 80\%). ${ }^{1} \mathrm{H}$ NMR $\left(400 \mathrm{MHz}, \mathrm{CDCl}_{3}\right) \delta$ $8.87\left(\mathrm{~s}, 4 \mathrm{H}, \mathrm{tpy}-H^{3^{\prime}, 5^{\prime}}\right), 8.76\left(\mathrm{ddd}, J=4.8,1.8,0.9 \mathrm{~Hz}, 4 \mathrm{H}, \mathrm{tpy}-H^{6,6^{\prime \prime}}\right), 8.73(\mathrm{dt}, J=8.0$, $\left.1.1 \mathrm{~Hz}, 4 \mathrm{H}, \mathrm{tpy}-H^{3,3 "}\right), 8.23\left(\mathrm{t}, J=1.7 \mathrm{~Hz}, 1 \mathrm{H}, \mathrm{Ph}-H^{\mathrm{A}}\right), 8.11(\mathrm{~d}, J=1.7 \mathrm{~Hz}, 2 \mathrm{H}$, Ph- $\left.H^{\mathrm{B}}\right), 7.92\left(\mathrm{ddd}, J=7.9,7.5,1.8 \mathrm{~Hz}, 4 \mathrm{H}, \mathrm{tpy}-H^{4,4 "}\right), 7.67-7.61\left(\mathrm{~m}, 2 \mathrm{H}, \mathrm{Ph}-H^{\mathrm{C}}\right)$, $7.38\left(\mathrm{ddd}, J=7.5,4.8,1.2 \mathrm{~Hz}, 4 \mathrm{H}, \mathrm{tpy}-H^{5,5^{\prime \prime}}\right), 6.88-6.82\left(\mathrm{~m}, 2 \mathrm{H}, \mathrm{Ph}-H^{\mathrm{D}}\right), 3.78(\mathrm{br}$, $\left.2 \mathrm{H},-\mathrm{NH}_{2}\right) .{ }^{13} \mathrm{C} \mathrm{NMR}\left(100 \mathrm{MHz}, \mathrm{CDCl}_{3}\right) \delta 156.26,156.02,150.50,149.14,146.35$, $142.84,140.11,136.85,130.72,128.42,126.19,124.31,123.81,121.42,119.39$, 115.43. ESI-MS (m/z): Calcd. for $\left[\mathrm{C}_{42} \mathrm{H}_{29} \mathrm{~N}_{7}+\mathrm{H}\right]^{+}$: 632.3. Found: 632.3.

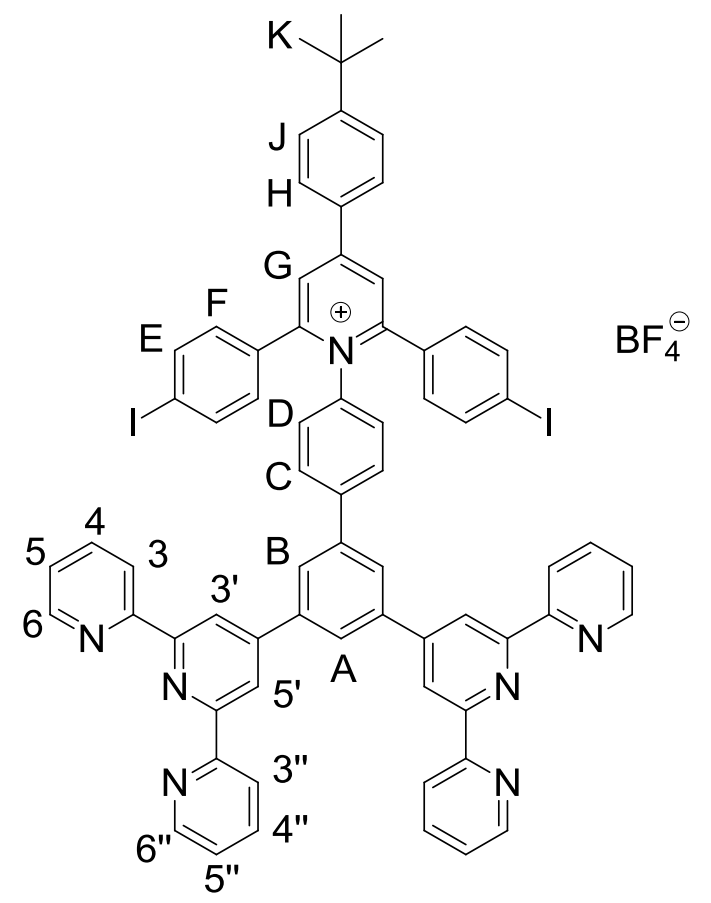

Compound 9. Anhydrous sodium acetate $(328 \mathrm{mg}, 4.0 \mathrm{mmol})$ and the pyrylium salt $\mathbf{1}^{6}$ (704 mg, $1.0 \mathrm{mmol})$ were added to a solution of $7(631 \mathrm{mg}, 1.0 \mathrm{mmol})$ in anhydrous ethanol $(25 \mathrm{~mL})$. The mixture was reflux for $8 \mathrm{~h}$ and then cooled to room temperature. The precipitate was filtered and washed with water and diethyl ether to give the product as a white solid $(1.07 \mathrm{~g}, 81 \%) .{ }^{1} \mathrm{H}$ NMR $\left(400 \mathrm{MHz}, \mathrm{DMSO}-d_{6}\right) \delta 8.72(\mathrm{~m}, 8 \mathrm{H}$, tpy- $H^{3^{\prime}, 5^{\prime}}$ and tpy- $\left.H^{6,6^{\prime \prime}}\right), 8.66\left(\mathrm{~s}, 2 \mathrm{H}, \mathrm{Ph}-H^{\mathrm{G}}\right), 8.60\left(\mathrm{~d}, J=7.8 \mathrm{~Hz}, 4 \mathrm{H}, \mathrm{tpy}-H^{3,3^{\prime \prime}}\right), 8.25$ 
$\left(\mathrm{m}, 3 \mathrm{H}, \mathrm{Ph}-H^{\mathrm{A}}\right.$ and $\left.\mathrm{Ph}-H^{\mathrm{H}}\right), 8.09\left(\mathrm{~s}, 2 \mathrm{H}, \mathrm{Ph}-H^{\mathrm{B}}\right), 8.05-7.97\left(\mathrm{~m}, 4 \mathrm{H}, \mathrm{tpy}-H^{4,4 "}\right), 7.84(\mathrm{~s}$, 2H, Ph- $\left.H^{\mathrm{C}}\right), 7.79\left(\mathrm{~d}, J=8.3 \mathrm{~Hz}, 4 \mathrm{H}, \mathrm{Ph}-H^{\mathrm{E}}\right), 7.69\left(\mathrm{~d}, J=8.5 \mathrm{~Hz}, 2 \mathrm{H}, \mathrm{Ph}-H^{\mathrm{J}}\right), 7.62(\mathrm{~s}$, 2H, Ph- $\left.H^{\mathrm{D}}\right), 7.51\left(\mathrm{~m}, 4 \mathrm{H}, \mathrm{tpy}-H^{5,5 "}\right), 7.30\left(\mathrm{~d}, J=8.6 \mathrm{~Hz}, 4 \mathrm{H}, \mathrm{Ph}-H^{\mathrm{F}}\right), 1.36(\mathrm{~s}, 9 \mathrm{H}$, tert-butyl- $\left.H^{\mathrm{K}}\right) .{ }^{13} \mathrm{C}$ NMR $\left(100 \mathrm{MHz}, \mathrm{DMSO}-d_{6}\right) \delta 156.09,155.85,155.37,150.82$, $149.72,149.30,140.53,140.20,139.90,139.02,138.07,137.92,137.60,132.93$, $132.10,130.95,129.84,129.75,129.13,127.81,127.15,126.59,125.41,125.00$, 121.51, 119.07, 99.98, 98.26, 35.4, 31.3. ESI-MS (m/z): Calcd. for $\left[\mathrm{C}_{69} \mathrm{H}_{50} \mathrm{I}_{2} \mathrm{~N}_{7}\right]^{+}$: 1230.2. Found: 1230.2.

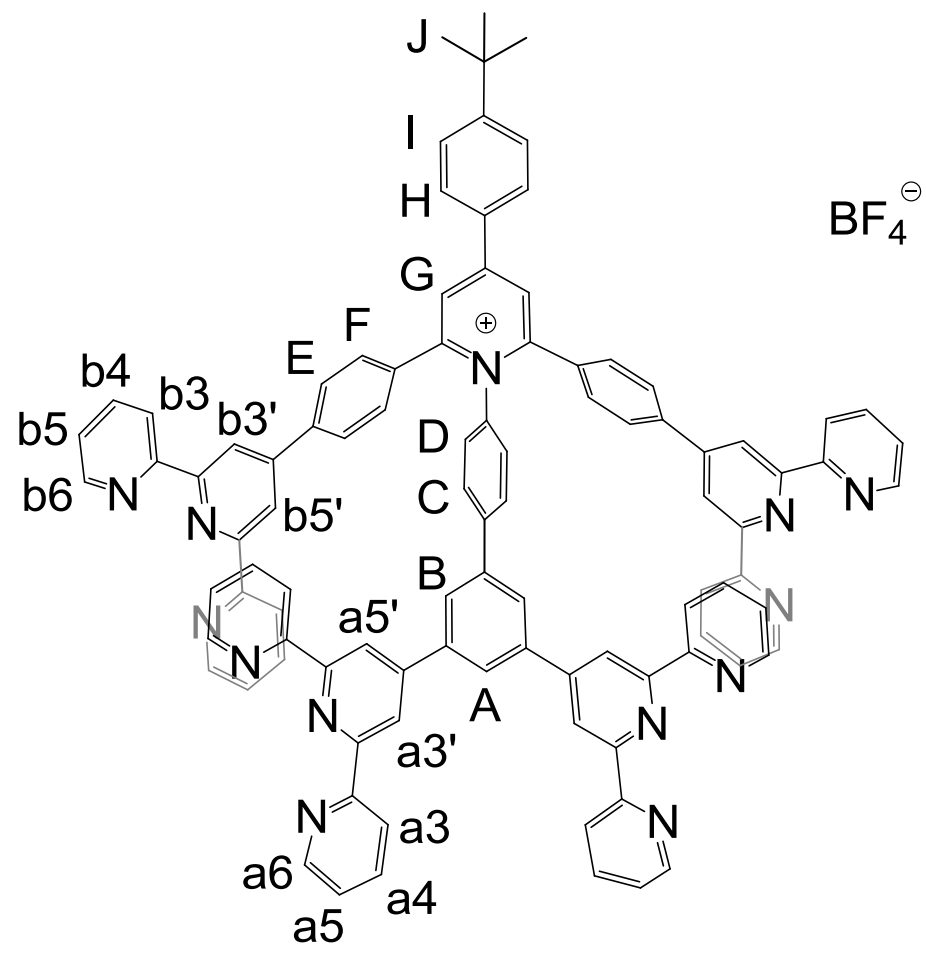

Ligand LA: To a solution of compound 9 (120.0 mg, $91.1 \mu \mathrm{mol}), \mathrm{Pd}\left(\mathrm{PPh}_{3}\right)_{2} \mathrm{Cl}_{2}(14.0$ mg, $20.0 \mu \mathrm{mol})$ and compound $2(81.8 \mathrm{mg}, 227.8 \mu \mathrm{mol})$ in DMSO $(25 \mathrm{~mL})$ under $\mathrm{N}_{2}$, aqueous $2 \mathrm{M} \mathrm{K}_{2} \mathrm{CO}_{3}(8 \mathrm{~mL})$ was added. The mixture was stirred at $80{ }^{\circ} \mathrm{C}$ for $30 \mathrm{~h}$ and then cooled to room temperature. The mixture was added into $200 \mathrm{ml}$ water and was extracted with $\mathrm{CHCl}_{3}$, and the combined organic phase was washed with brine and 
dried over $\mathrm{Na}_{2} \mathrm{SO}_{4}$. After removal of solvent under vacuum, the residue was purified by column chromatography on $\mathrm{Al}_{2} \mathrm{O}_{3}$. The polarity of eluent was increased from chloroform/acetone $100 / 0$ to $0 / 100$, then a mixture of acetone/acetonitrile, $70 / 30$ to 0/100 and finally a mixture of acetonitrile/water $/ \mathrm{NH}_{4} \mathrm{BF}_{4}$ to afford $\mathbf{L A}$ as a white solid (61\% yield). ${ }^{1} \mathrm{H}$ NMR (400 MHz, $\left.\mathrm{CDCl}_{3}\right) \delta 8.55-8.51\left(\mathrm{~m}, 4 \mathrm{H}, \mathrm{tpy}-H^{\mathrm{a} 3,3 "}\right), 8.45$ $-8.40\left(\mathrm{~m}, 4 \mathrm{H}, \mathrm{tpy}-H^{\mathrm{b} 3,3^{\prime \prime}}\right), 8.40-8.32\left(\mathrm{~m}, 8 \mathrm{H}, \mathrm{tpy}-H^{\mathrm{a} 3^{\prime}, 5^{\prime}}\right.$ and tpy- $\left.H^{\mathrm{b} 3^{\prime}, 5^{\prime}}\right), 8.28(\mathrm{~m}, 8 \mathrm{H}$, tpy- $H^{\mathrm{a} 6,6 "}$ and tpy- $\left.H^{\mathrm{b} 6,6^{\prime \prime}}\right), 7.98-7.95\left(\mathrm{~m}, 4 \mathrm{H}, \mathrm{Ph}-H^{\mathrm{G}}\right.$ and $\left.\mathrm{Ph}-H^{\mathrm{H}}\right), 7.94-7.90(\mathrm{~m}, 4 \mathrm{H}$, $\left.\mathrm{Ph}-H^{\mathrm{E}}\right), 7.84-7.75\left(\mathrm{~m}, 8 \mathrm{H}, \mathrm{Ph}-H^{\mathrm{B}}, \mathrm{Ph}-H^{\mathrm{C}}\right.$ and $\left.\mathrm{Ph}-H^{\mathrm{F}}\right), 7.72-7.67\left(\mathrm{~m}, 4 \mathrm{H}, \mathrm{tpy}-H^{\mathrm{b} 4,4^{\prime \prime}}\right)$, 7.60-7.54 (m, 9H, Ph- $H^{\mathrm{A}}, \mathrm{Ph}-H^{\mathrm{D}}, \mathrm{Ph}-H^{\mathrm{I}}$ and tpy- $\left.H^{\mathrm{a} 4,4 "}\right), 7.23-7.15\left(\mathrm{~m}, 4 \mathrm{H}, \mathrm{tpy}-H^{\mathrm{b}, 5 "}\right)$, $7.14-7.04\left(\mathrm{~m}, 4 \mathrm{H}\right.$, tpy- $\left.H^{\mathrm{a} 5,5^{\prime \prime}}\right), 1.36\left(\mathrm{~s}, 9 \mathrm{H}\right.$, tert-butyl- $\left.H^{\mathrm{J}}\right) .{ }^{13} \mathrm{C}$ NMR $(100 \mathrm{MHz}$, $\left.\mathrm{CDCl}_{3}\right) \delta 156.46,156.45,155.95,155.91,155.75,155.71,149.28,149.08,149.01$ $148.95,148.27,141.62,140.32,140.00,138.72,136.77,136.61,133.65,132.14$ $131.92,131.24,130.80,129.32,128.43,127.94,127.47,126.87,126.36,126.08$, $123.82,123.65,121.35,121.21,121.11,118.98,118.74,35.17,31.10$. MALDI-TOF MS (m/z): Calcd. for $\left[\mathrm{C}_{105} \mathrm{H}_{82} \mathrm{~N}_{13} \mathrm{O}\right]^{+}$1440.6. Found: 1440.6.

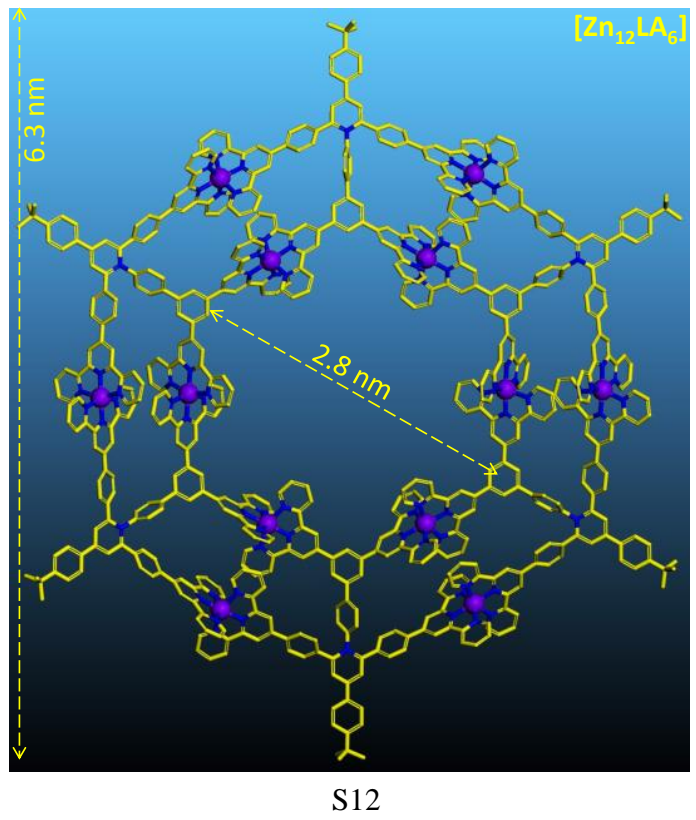


Complex [ $\left.\mathbf{Z n}_{12} \mathbf{L A} \mathbf{A}_{6}\right]$ : To a solution of ligand $\mathbf{L A}(6.0 \mathrm{mg}, 3.9 \mu \mathrm{mol})$ in $\mathrm{CHCl}_{3}(3 \mathrm{~mL})$, a solution of $\mathrm{Zn}\left(\mathrm{NO}_{3}\right)_{2} \bullet 6 \mathrm{H}_{2} \mathrm{O}(2.3 \mathrm{mg}, 7.9 \mu \mathrm{mol})$ in $\mathrm{MeOH}(9 \mathrm{~mL})$ was added. The mixture was stirred at $50{ }^{\circ} \mathrm{C}$ for $8 \mathrm{~h}$ and then cooled to room temperature. Upon addition of $\mathrm{NH}_{4} \mathrm{PF}_{6}(200 \mathrm{mg}$ ), a precipitate was formed and washed with water to give a white product (yield: 90\%). ESI-MS (m/z): $1578.0\left[{\mathrm{M}-8 \mathrm{PF}_{6}}^{-}\right]^{8+}(\mathrm{calcd} \mathrm{m} / \mathrm{z}: 1578.0)$, $1386.6\left[{\mathrm{M}-9 \mathrm{PF}_{6}}^{-}\right]^{9+}($ calcd $m / z: 1386.6), 1233.6\left[{\mathrm{M}-10 \mathrm{PF}_{6}^{-}}^{-10+}(\right.$ calcd $\mathrm{m} / \mathrm{z}: 1233.6)$, $1108.1\left[\mathrm{M}^{-11 \mathrm{PF}_{6}}{ }^{-}\right]^{11+}($ calcd $m / z: 1108.1), 1003.6\left[{\mathrm{M}-12 \mathrm{PF}_{6}^{-}}^{-12+}(\mathrm{calcd} m / z: 1003.6)\right.$, $915.2\left[\mathrm{M}-13 \mathrm{PF}_{6}^{-}\right]^{13+}($ calcd $\mathrm{m} / z: 915.2), 839.6\left[{\mathrm{M}-14 \mathrm{PF}_{6}}^{-}\right]^{14+}($ calcd $\mathrm{m} / z: 839.6)$, $773.8\left[\mathrm{M}-15 \mathrm{PF}_{6}^{-}\right]^{15+}($ calcd $m / z: 773.8), 716.5\left[{\mathrm{M}-16 \mathrm{PF}_{6}^{-}}^{-16+}(\right.$ calcd $m / z: 716.5)$, $665.9\left[\mathrm{M}_{-17 \mathrm{PF}_{6}}^{-}\right]^{17+}(\operatorname{calcd} \mathrm{m} / z: 665.9)$ and $620.8\left[\mathrm{M}-18 \mathrm{PF}_{6}^{-}\right]^{18+}($ calcd $m / z: 620.8)$.

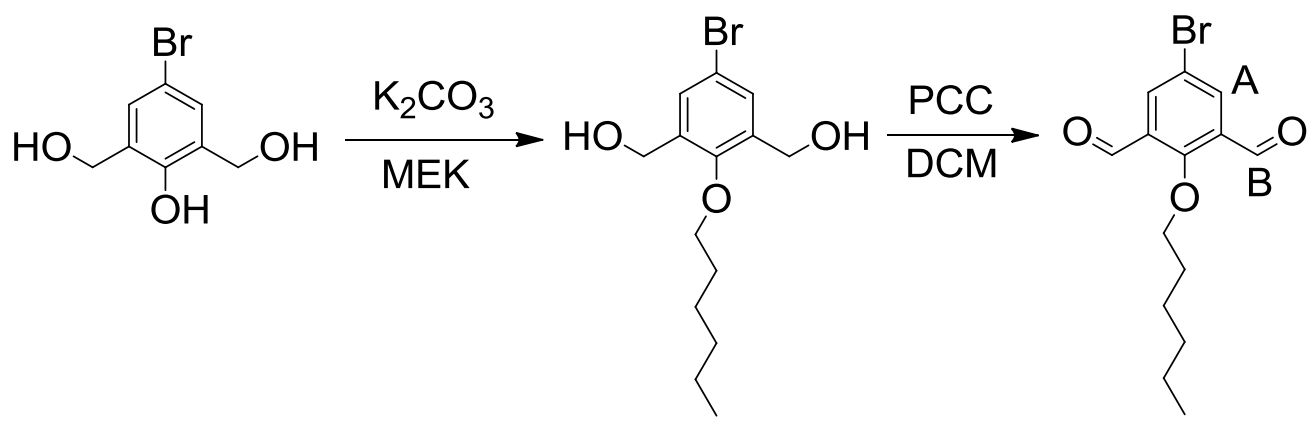

Compound 4. A mixture of 4-bromo-2,6-bis(hydroxymethyl)phenol ${ }^{7}$ (6.0 g, 25.9 mmol), 1-bromohexane (5.1 g, $31.1 \mathrm{mmol}), \mathrm{K}_{2} \mathrm{CO}_{3}(7.2 \mathrm{~g}, 52.0 \mathrm{mmol})$ and methyl ethyl ketone $(200 \mathrm{~mL})$ was refluxed under $\mathrm{N}_{2}$ for $24 \mathrm{~h}$. The mixture was cooled to room temperature, filtrated and dried under vacuum. The white residue, PCC (11.8 g, $75 \mathrm{mmol})$ and celite $(10 \mathrm{~g})$ were dissolved in DCM (200 $\mathrm{ml})$, and the mixture was stirred for $20 \mathrm{~h}$ at room temperature. The solution was filtered and poured onto silica gel column with DCM as eluent to afford 
5-bromo-2-(hexyloxy)benzene-1,3-dialdehyde as a white solid (86\% yield). ${ }^{1} \mathrm{H}$ NMR (400 MHz, $\left.\mathrm{CDCl}_{3}\right) \delta 10.36\left(\mathrm{~s}, 2 \mathrm{H}, \mathrm{Ph}-H^{\mathrm{B}}\right), 8.20\left(\mathrm{~s}, 2 \mathrm{H}, \mathrm{Ph}-H^{\mathrm{A}}\right), 4.14(\mathrm{t}, J=6.4$ $\mathrm{Hz}, 2 \mathrm{H}), 1.95-1.87(\mathrm{~m}, 2 \mathrm{H}), 1.56-1.45(\mathrm{~m}, 2 \mathrm{H}), 1.40-1.35(\mathrm{~m}, 4 \mathrm{H}), 0.97-0.89(\mathrm{t}, J$ $=6.4 \mathrm{~Hz}, 3 \mathrm{H}) .{ }^{13} \mathrm{C} \mathrm{NMR}\left(100 \mathrm{MHz}, \mathrm{CDCl}_{3}\right) \delta 187.13,139.03,137.24,131.76,118.29$, $81.04,31.48,29.85,25.45,22.49,13.94$.

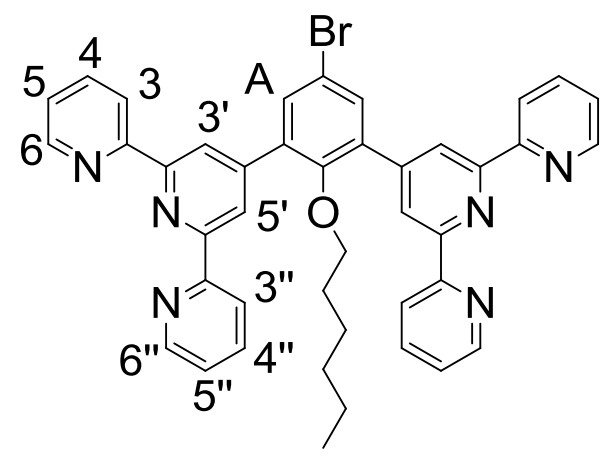

Compound 6. To a solution of $\mathrm{NaOH}$ powder $(6.24 \mathrm{~g}, 156.0 \mathrm{mmol})$ in $\mathrm{EtOH}(350 \mathrm{ml})$, 5-bromo-2-(hexyloxy)benzene-1,3-dialdehyde $\quad\left(\begin{array}{llll}4.0 & \mathrm{~g}, & 13.0 & \mathrm{mmol})\end{array}\right.$ and 2-acetylpyridine $(7.5 \mathrm{~g}, 62 \mathrm{mmol})$ were added. After stirring at room temperature for $20 \mathrm{~h}$, aqueous $\mathrm{NH}_{3} \cdot \mathrm{H}_{2} \mathrm{O}(150 \mathrm{~mL})$ was added and the mixture was refluxed for $40 \mathrm{~h}$. Upon cooling to room temperature, the precipitate was filtered and washed with cold ethanol to give 6 as a white solid $(4.8 \mathrm{~g}, 51 \%) .{ }^{1} \mathrm{H} \mathrm{NMR}\left(400 \mathrm{MHz}, \mathrm{CDCl}_{3}\right) \delta 8.80(\mathrm{~s}$, $\left.4 \mathrm{H}, \mathrm{tpy}-H^{3^{\prime}, 5^{\prime}}\right), 8.77\left(\mathrm{ddd}, J=4.8,1.8,0.9 \mathrm{~Hz}, 4 \mathrm{H}, \mathrm{tpy}-H^{6,6 "}\right), 8.70(\mathrm{dt}, J=8.0,1.0 \mathrm{~Hz}$, 4H, tpy- $\left.H^{3,3 "}\right), 7.91\left(\mathrm{td}, J=7.7,1.8 \mathrm{~Hz}, 4 \mathrm{H}, \mathrm{tpy}-H^{4,4 "}\right), 7.80\left(\mathrm{~s}, 2 \mathrm{H}, \mathrm{Ph}-H^{\mathrm{A}}\right), 7.38(\mathrm{ddd}$, $\left.J=7.5,4.8,1.2 \mathrm{~Hz}, 4 \mathrm{H}, \mathrm{tpy}-H^{5,5 "}\right), 3.37(\mathrm{t}, J=6.4 \mathrm{~Hz}, 2 \mathrm{H}), 1.24-1.17(\mathrm{dd}, J=9.1$, $6.2 \mathrm{~Hz}, 2 \mathrm{H}), 0.92(\mathrm{~s}, 2 \mathrm{H}), 0.83-0.66(\mathrm{~m}, 4 \mathrm{H}), 0.55-0.41(\mathrm{t}, J=6.8 \mathrm{~Hz}, 3 \mathrm{H}) .{ }^{13} \mathrm{C}$ NMR $\left(100 \mathrm{MHz}, \mathrm{CDCl}_{3}\right) \delta 156.10,155.58,153.98,149.20,147.08,136.80,135.89$, 133.66, 123.82, 121.45, 121.25, 117.05, 74.33, 31.29, 29.70, 25.39, 22.11, 13.77 . ESI-MS (m/z): Calcd. for $\left[\mathrm{C}_{42} \mathrm{H}_{35} \mathrm{BrN}_{6} \mathrm{O}+\mathrm{H}\right]^{+}:$719.2. Found: 719.2. 


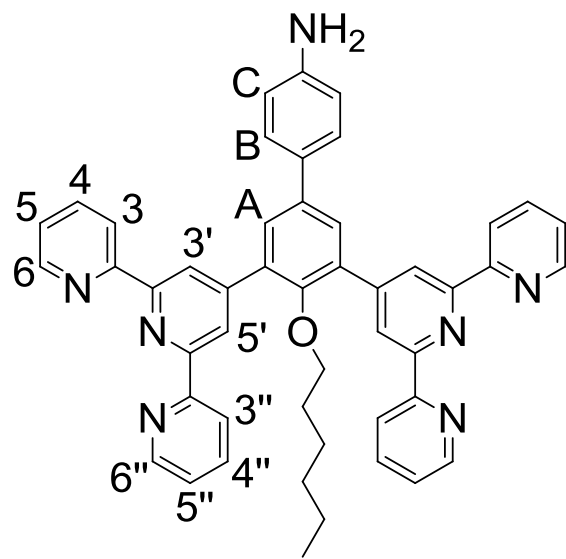

Compound 8. To a flask containing a degassed solution of compound 6 (720 mg, 1 mmol), 4-(4,4,5,5-tetramethyl-1,3,2-dioxaborolan-2-yl)aniline (330 mg, $1.5 \mathrm{mmol})$ in toluene $(30 \mathrm{~mL})$, an aqueous solution of $2 \mathrm{M} \mathrm{K}_{2} \mathrm{CO}_{3}(15 \mathrm{~mL})$ was added. The $\mathrm{Pd}\left(\mathrm{PPh}_{3}\right)_{2} \mathrm{Cl}_{2}(70 \mathrm{mg}, 0.1 \mathrm{mmol})$ was added into the mixture under $\mathrm{N}_{2}$. The mixture was stirred at $90^{\circ} \mathrm{C}$ for $20 \mathrm{~h}$ and then cooled to room temperature. The aqueous phase was extracted with $\mathrm{CH}_{2} \mathrm{Cl}_{2}$, and the combined organic phase was washed with brine and dried with anhydrous $\mathrm{Na}_{2} \mathrm{SO}_{4}$. After removal of solvent under vacuum, the crude was purified by column chromatography on silica gel with chloroform: ethanol (100:1) as eluent to afford the product as a white solid (636 mg, 87\%). ${ }^{1} \mathrm{H}$ NMR (400 MHz, $\left.\mathrm{CDCl}_{3}\right) \delta 8.85\left(\mathrm{~s}, 4 \mathrm{H}, \mathrm{tpy}-H^{3^{\prime}, 5^{\prime}}\right), 8.76\left(\mathrm{ddd}, J=4.8,1.6,0.8 \mathrm{~Hz}, 4 \mathrm{H}\right.$, tpy- $\left.H^{6,6 "}\right), 8.70(\mathrm{~d}$, $\left.J=8.0 \mathrm{~Hz}, 4 \mathrm{H}, \mathrm{tpy}-H^{3,3 "}\right), 7.89\left(\mathrm{td}, J=7.7,1.8 \mathrm{~Hz}, 4 \mathrm{H}, \mathrm{tpy}-H^{4,4 "}\right), 7.78\left(\mathrm{~s}, 2 \mathrm{H}, \mathrm{Ph}-H^{\mathrm{A}}\right)$, $7.52\left(\mathrm{~d}, J=8.5 \mathrm{~Hz}, 2 \mathrm{H}, \mathrm{Ph}-H^{\mathrm{B}}\right), 7.35\left(\mathrm{ddd}, J=7.5,4.8,1.1 \mathrm{~Hz}, 4 \mathrm{H}, \mathrm{tpy}-H^{5,5 "}\right), 6.78(\mathrm{~d}$, $\left.J=8.5 \mathrm{~Hz}, 2 \mathrm{H}, \mathrm{Ph}-H^{\mathrm{C}}\right), 3.76\left(\mathrm{br}, 2 \mathrm{H},-N H_{2}\right), 3.40(\mathrm{t}, J=6.1 \mathrm{~Hz}, 2 \mathrm{H}), 1.28-1.10(\mathrm{~m}$, 2H), $0.97-0.89(\mathrm{~m}, 2 \mathrm{H}), 0.82-0.68(\mathrm{~m}, 4 \mathrm{H}), 0.58-0.43(\mathrm{t}, J=6.8 \mathrm{~Hz}, 3 \mathrm{H}) .{ }^{13} \mathrm{C}$ NMR $\left(100 \mathrm{MHz}, \mathrm{CDCl}_{3}\right) \delta 156.42,155.41,153.38,149.19,148.77,145.95,137.51$, $136.73,134.10,130.41,129.08,128.12,123.65,121.82,121.26,115.41,74.22,31.36$, 29.78, 25.47, 22.15, 13.79. ESI-MS (m/z): Calcd. for $\left[\mathrm{C}_{48} \mathrm{H}_{41} \mathrm{~N}_{7} \mathrm{O}+\mathrm{H}\right]^{+}:$732.3. Found: 
732.3 .

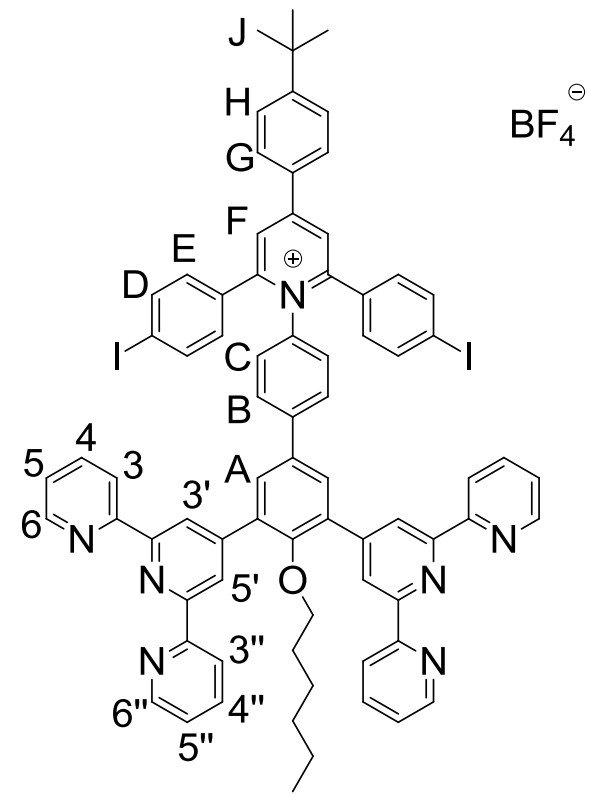

Compound 10. Anhydrous sodium acetate $(328 \mathrm{mg}, 4.0 \mathrm{mmol})$ and the pyrylium salt 1 (704 mg, $1.0 \mathrm{mmol})$ were added to a solution of $\mathbf{8}(731 \mathrm{mg}, 1.0 \mathrm{mmol})$ in dry ethanol (20 mL). The mixture was reflux for $10 \mathrm{~h}$ and then cooled to room temperature. The precipitate was filtered and washed with water and diethyl ether to give the product as a white solid (1.06 g, 75\%). ${ }^{1} \mathrm{H}$ NMR (400 MHz, DMSO- $\left.d_{6}\right) \delta 8.81-8.73(\mathrm{~m}, 8 \mathrm{H}$, tpy- $H^{3^{\prime}, 5^{\prime}}$ and tpy- $\left.H^{6,6 "}\right), 8.69\left(\mathrm{~d}, J=7.9 \mathrm{~Hz}, 4 \mathrm{H}, \mathrm{tpy}-H^{3,3 "}\right), 8.65\left(\mathrm{~s}, 2 \mathrm{H}, \mathrm{Ph}-H^{\mathrm{F}}\right), 8.27(\mathrm{~d}$, $\left.J=8.6 \mathrm{~Hz}, 2 \mathrm{H}, \mathrm{Ph}-H^{\mathrm{G}}\right), 8.04\left(\mathrm{td}, J=7.8,1.7 \mathrm{~Hz}, 4 \mathrm{H}, \mathrm{tpy}-H^{4,4 "}\right), 7.94\left(\mathrm{~s}, 2 \mathrm{H}, \mathrm{Ph}-H^{\mathrm{A}}\right)$, $7.88\left(\mathrm{~d}, J=8.5 \mathrm{~Hz}, 2 \mathrm{H}, \mathrm{Ph}-H^{\mathrm{B}}\right), 7.78\left(\mathrm{~d}, J=8.4 \mathrm{~Hz}, 4 \mathrm{H}, \mathrm{Ph}-H^{\mathrm{D}}\right), 7.67(\mathrm{~d}, J=8.6 \mathrm{~Hz}$, $\left.2 \mathrm{H}, \mathrm{Ph}-H^{\mathrm{H}}\right), 7.58\left(\mathrm{~d}, J=8.6 \mathrm{~Hz}, 2 \mathrm{H}, \mathrm{Ph}-H^{\mathrm{C}}\right), 7.54-7.48\left(\mathrm{~m}, 4 \mathrm{H}, \mathrm{tpy}-H^{5,5^{\prime \prime}}\right), 7.26(\mathrm{~d}, J$ $\left.=8.3 \mathrm{~Hz}, 4 \mathrm{H}, \mathrm{Ph}-H^{\mathrm{E}}\right), 3.25(\mathrm{t}, J=5.5 \mathrm{~Hz}, 2 \mathrm{H}), 1.35\left(\mathrm{~s}, 9 \mathrm{H}\right.$, tert-butyl- $\left.H^{\mathrm{J}}\right), 1.10-1.00$ $(\mathrm{m}, 2 \mathrm{H}), 0.85-0.75(\mathrm{~m}, 2 \mathrm{H}), 0.63-0.55(\mathrm{~m}, 4 \mathrm{H}), 0.36(\mathrm{t}, J=6.8 \mathrm{~Hz}, 3 \mathrm{H}) .{ }^{13} \mathrm{C} \mathrm{NMR}$ $\left(100 \mathrm{MHz}, \mathrm{DMSO}-d_{6}\right) \delta 156.38,155.94,155.88,155.52,154.80,149.81,147.78$, $139.91,138.71,137.91,137.56,134.65,133.00,132.07,131.98,131.88,131.01$, $129.74,129.44,129.27,129.15,127.29,127.10,125.39,124.92,121.55,121.35$, 
98.22, 74.24, 35.37, 31.28, 31.08, 29.58, 25.38, 21.99, 13.96. ESI-MS (m/z): Calcd. for $\left[\mathrm{C}_{75} \mathrm{H}_{62} \mathrm{I}_{2} \mathrm{~N}_{7} \mathrm{O}\right]^{+}:$1330.3. Found: 1330.2.

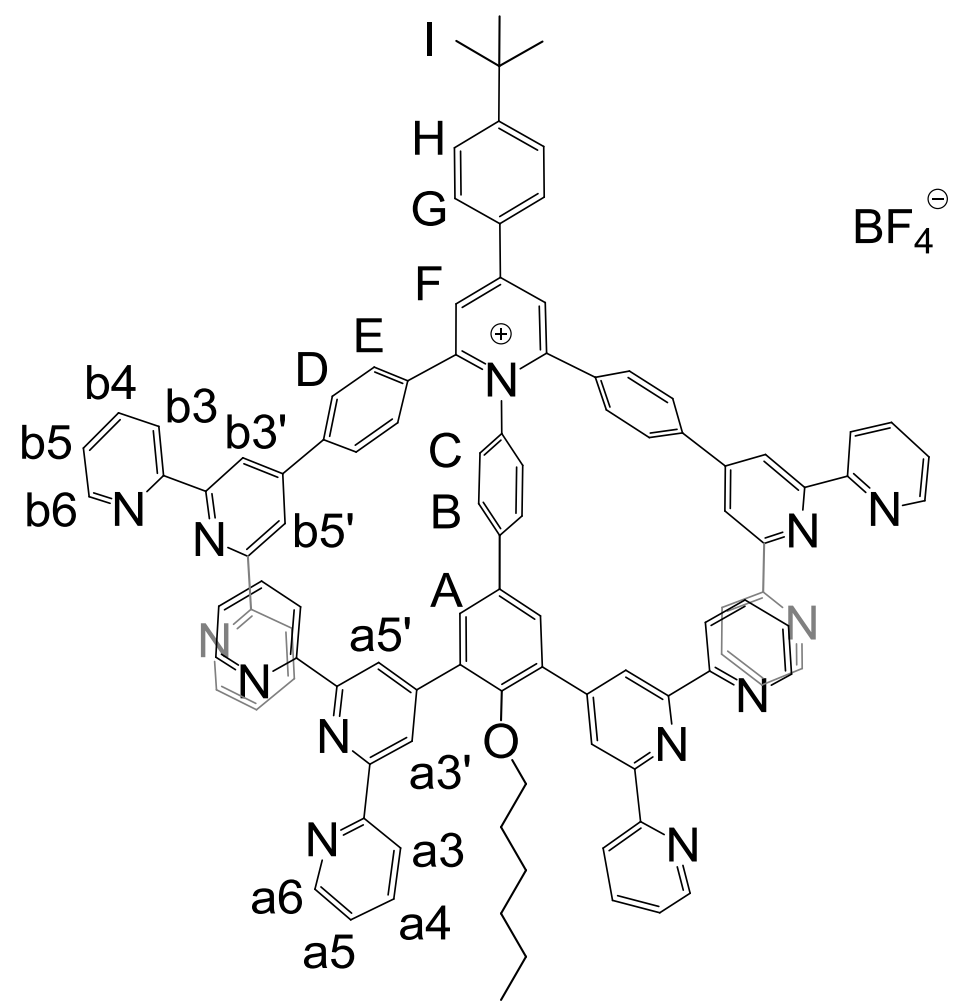

Ligand LB: To a solution of compound $10(150.0 \mathrm{mg}, 105.9 \mu \mathrm{mol}), \mathrm{Pd}\left(\mathrm{PPh}_{3}\right)_{2} \mathrm{Cl}_{2}$ (14.0 mg, $20.0 \mu \mathrm{mol})$ and compound $2(95.0 \mathrm{mg}, 264.6 \mu \mathrm{mol})$ in DMSO (30 mL) under nitrogen, aqueous $2 \mathrm{M} \mathrm{K}_{2} \mathrm{CO}_{3}(8 \mathrm{~mL})$ was added. The mixture was stirred at 80 ${ }^{\circ} \mathrm{C}$ for $20 \mathrm{~h}$ and then cooled to room temperature. The mixture was added into $250 \mathrm{ml}$ water and was extracted with $\mathrm{CHCl}_{3}$, and the combined organic phase was washed with brine and dried over $\mathrm{Na}_{2} \mathrm{SO}_{4}$. After removal of solvent under vacuum, the residue was purified by column chromatography on $\mathrm{Al}_{2} \mathrm{O}_{3}$. The polarity of eluent was increased from chloroform/acetone $100 / 0$ to $0 / 100$, then a mixture of acetone/acetonitrile, $60 / 40$ to $0 / 100$ and finally a mixture of acetonitrile/water/ $\mathrm{NH}_{4} \mathrm{BF}_{4}$ to afford $\mathbf{L B}$ as a white solid (64\% yield). ${ }^{1} \mathrm{H}$ NMR (400 $\left.\mathrm{MHz}, \mathrm{CDCl}_{3}\right) \delta 8.69\left(\mathrm{~s}, 4 \mathrm{H}\right.$, tpy- $\left.H^{\mathrm{a} 3^{\prime}, 5^{\prime}}\right), 8.66\left(\mathrm{~s}, 4 \mathrm{H}, \mathrm{tpy}-H^{\mathrm{b} 3^{\prime}, 5^{\prime}}\right), 8.64-8.60(\mathrm{~d}, J=6.6$ 
$\mathrm{Hz}, 12 \mathrm{H}, \mathrm{tpy}-H^{\mathrm{a} 6,6 "}, \operatorname{tpy}-H^{\mathrm{a} 3,3 "}$ and tpy- $\left.H^{\mathrm{b} 6,6 "}\right), 8.59\left(\mathrm{~d}, J=8.0 \mathrm{~Hz}, 4 \mathrm{H}, \mathrm{tpy}-H^{\mathrm{b} 3,3 "}\right), 8.26$ $\left(\mathrm{s}, 2 \mathrm{H}, \mathrm{Ph}-H^{\mathrm{F}}\right), 7.98\left(\mathrm{~d}, J=8.5 \mathrm{~Hz}, 2 \mathrm{H}, \mathrm{Ph}-H^{\mathrm{G}}\right), 7.92\left(\mathrm{~d}, J=8.4 \mathrm{~Hz}, 4 \mathrm{H}, \mathrm{Ph}-H^{\mathrm{D}}\right), 7.88$ $-7.78\left(\mathrm{~m}, 14 \mathrm{H}, \mathrm{Ph}-H^{\mathrm{E}}, \mathrm{Ph}-H^{\mathrm{B}}\right.$, tpy- $H^{\mathrm{a} 4,4^{\prime \prime}}$ and tpy- $\left.H^{\mathrm{b} 4,4 "}\right), 7.71\left(\mathrm{~s}, 2 \mathrm{H}, \mathrm{Ph}-H^{\mathrm{A}}\right), 7.64(\mathrm{~d}$, $\left.J=8.6 \mathrm{~Hz}, 2 \mathrm{H}, \mathrm{Ph}-H^{\mathrm{H}}\right), 7.60\left(\mathrm{~d}, J=8.1 \mathrm{~Hz}, 2 \mathrm{H}, \mathrm{Ph}-H^{\mathrm{C}}\right), 7.35-7.23(\mathrm{~m}, 8 \mathrm{H}$, tpy- $H^{\mathrm{a} 5,5^{\prime \prime}}$ and tpy- $\left.H^{\mathrm{b} 5,5^{\prime \prime}}\right), 3.27(\mathrm{t}, J=6.0 \mathrm{~Hz}, 2 \mathrm{H}), 1.40\left(\mathrm{~s}, 9 \mathrm{H}\right.$, tert-butyl- $\left.H^{\mathrm{I}}\right), 1.19-$ $1.05(\mathrm{~m}, 2 \mathrm{H}), 0.90-0.80(\mathrm{~m}, 2 \mathrm{H}), 0.79-0.62(\mathrm{~m}, 4 \mathrm{H}), 0.45(\mathrm{t}, J=6.8 \mathrm{~Hz}, 3 \mathrm{H}) .{ }^{13} \mathrm{C}$ NMR $\left(100 \mathrm{MHz}, \mathrm{CDCl}_{3}\right) \delta 156.94,156.48,156.46,156.17,156.02,155.89,155.36$, $155.07,149.12,149.10,148.50,147.94,141.27,140.37,138.16,136.70,136.61$, $134.37,134.33,133.57,131.24,130.66,129.68,129.20,128.39,127.54,126.88$ $126.12,123.78,123.68,123.58,121.56,121.38,121.21,121.15,118.90,74.23,67.09$, 35.16, 31.27, 31.09, 29.63, 25.32, 22.07, 13.72. MALDI-TOF MS (m/z): Calcd. for $\left[\mathrm{C}_{105} \mathrm{H}_{82} \mathrm{~N}_{13} \mathrm{O}\right]^{+}$1540.7. Found: 1540.8 .

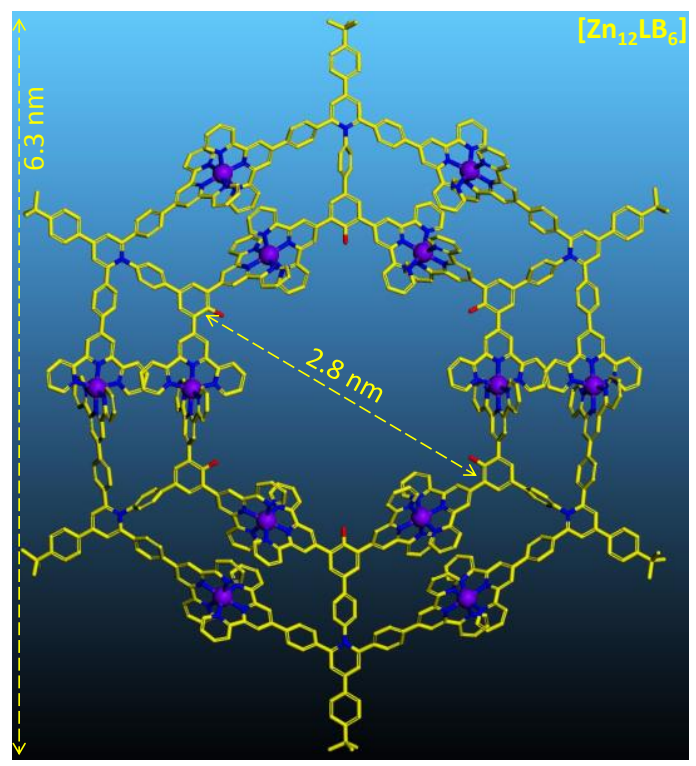

Complex $\left[\mathbf{Z n}_{12} \mathbf{L B} \mathbf{B}_{\mathbf{6}}\right]$ : To a solution of ligand $\mathbf{L B}(6.8 \mathrm{mg}, 4.2 \mu \mathrm{mol})$ in $\mathrm{CHCl}_{3}(1 \mathrm{~mL})$, a solution of $\mathrm{Zn}\left(\mathrm{NO}_{3}\right)_{2} \cdot 6 \mathrm{H}_{2} \mathrm{O}(2.5 \mathrm{mg}, 8.4 \mu \mathrm{mol})$ in $\mathrm{MeOH}(3 \mathrm{~mL})$ was added. The mixture was stirred at $55{ }^{\circ} \mathrm{C}$ for $8 \mathrm{~h}$ and then cooled to room temperature. Upon 
addition of $\mathrm{NH}_{4} \mathrm{PF}_{6}(200 \mathrm{mg}$ ), a precipitate was formed and washed with water to give a white product (yield: $92 \%) .{ }^{1} \mathrm{H}$ NMR $\left(400 \mathrm{MHz}, \mathrm{CD}_{3} \mathrm{CN}\right) \delta 9.18\left(\mathrm{~s}, 4 \mathrm{H}, \mathrm{tpy}-H^{\mathrm{a} 3^{\prime}, 5^{\prime}}\right)$, $9.07\left(\mathrm{~s}, 4 \mathrm{H}, \mathrm{tpy}-H^{\mathrm{b} 3^{\prime}, 5^{\prime}}\right), 8.68\left(\mathrm{~m}, \mathrm{tpy}-H^{\mathrm{a} 3,3^{\prime \prime}}\right.$ and $\left.\mathrm{Ph}-H^{\mathrm{F}}\right), 8.60\left(\mathrm{~m}, 4 \mathrm{H}, \mathrm{tpy}-H^{\mathrm{b} 3,3^{\prime \prime}}\right), 8.42$ $\left(\mathrm{m}, 4 \mathrm{H}, \mathrm{Ph}-H^{\mathrm{D}}\right), 8.27\left(\mathrm{~m}, 2 \mathrm{H}, \mathrm{Ph}-H^{\mathrm{G}}\right), 8.22\left(\mathrm{~s}, 2 \mathrm{H}, \mathrm{Ph}-H^{\mathrm{A}}\right), 8.02\left(\mathrm{~m}, 4 \mathrm{H}, \mathrm{Ph}-H^{\mathrm{E}}\right), 7.94$ (br, 4H, Ph- $H^{\mathrm{B}}$ and $\left.\mathrm{Ph}-H^{\mathrm{C}}\right), 7.87\left(\mathrm{~m}, 2 \mathrm{H}, \mathrm{Ph}-H^{\mathrm{H}}\right), 7.79\left(\mathrm{~m}, 8 \mathrm{H}\right.$, tpy- $H^{\mathrm{a} 4,4^{\prime \prime}}$ and tpy- $\left.H^{\mathrm{a} 6,6 "}\right), 7.73-7.65\left(\mathrm{~m}, 4 \mathrm{H}, \mathrm{tpy}-H^{\mathrm{b} 4,4 "}\right), 7.63\left(\mathrm{~m}, 4 \mathrm{H}, \mathrm{tpy}-H^{\mathrm{b} 6,6 "}\right), 7.18-7.10(\mathrm{~m}$, 4H, tpy- $\left.H^{\mathrm{a} 5,5^{\prime}}\right), 6.90\left(\mathrm{~s}, 4 \mathrm{H}, \mathrm{tpy}-H^{\mathrm{b} 5,5^{\prime}}\right), 3.55(\mathrm{br}, 2 \mathrm{H}), 1.50\left(\mathrm{~s}, 9 \mathrm{H}\right.$, tert-butyl- $\left.H^{\mathrm{I}}\right), 1.28$ $-1.20(\mathrm{~m}, 2 \mathrm{H}), 1.13-1.05(\mathrm{~m}, 2 \mathrm{H}), 0.75-0.66(\mathrm{~m}, 2 \mathrm{H}), 0.57-0.42(\mathrm{~m}, 2 \mathrm{H}), 0.16-$ $0.06(\mathrm{~m}, 3 \mathrm{H}) .{ }^{13} \mathrm{C} \mathrm{NMR}\left(100 \mathrm{MHz}, \mathrm{CD}_{3} \mathrm{CN}\right) \delta 156.08,154.69,152.98,149.81,149.14$ $148.07,147.89,147.72,141.08,138.00,137.89,135.62,132.67,132.25,131.22$, $131.09,130.69,129.32,128.71,128.39,127.60,127.18,124.69,123.50,123.44$, $123.09,121.92,35.01,31.10,30.31,30.21,25.91,21.80,12.75$. ESI-MS $(\mathrm{m} / \mathrm{z})$ : $1653.0\left[{\mathrm{M}-8 \mathrm{PF}_{6}^{-}}^{-}\right]^{8+}($ calcd $m / z: 1653.0), 1452.2\left[{\mathrm{M}-9 \mathrm{PF}_{6}^{-}}^{-9+}(\right.$ calcd $\mathrm{m} / z: 1452.2)$, $1293.5\left[\mathrm{M}-10 \mathrm{PF}_{6}^{-}\right]^{10+}(\operatorname{calcd} m / z: 1293.5), 1162.7\left[{\mathrm{M}-11 \mathrm{PF}_{6}}^{-}\right]^{11+}(\operatorname{calcd} \mathrm{m} / z: 1162.7)$, $1053.8\left[{\mathrm{M}-12 \mathrm{PF}_{6}^{-}}^{-12+}(\right.$ calcd $m / z: 1053.8), 961.5\left[\mathrm{M}-13 \mathrm{PF}_{6}^{-}\right]^{13+}($ calcd $m / z: 961.5)$, $882.5\left[\mathrm{M}-14 \mathrm{PF}_{6}^{-}\right]^{14+}($ calcd $m / z: 882.5), 814.0\left[{\mathrm{M}-15 \mathrm{PF}_{6}}^{-}\right]^{15+}($ calcd $m / z: 814.0)$, $754.1\left[\mathrm{M}-16 \mathrm{PF}_{6}^{-}\right]^{16+}($ calcd $m / z: 754.1), 701.2\left[{\mathrm{M}-17 \mathrm{PF}_{6}}^{-}\right]^{17+}($ calcd $m / z: 701.2)$, $654.2\left[\mathrm{M}-18 \mathrm{PF}_{6}^{-}\right]^{18+}($ calcd $\mathrm{m} / z: 654.2), 612.2\left[\mathrm{M}^{19} 19 \mathrm{PF}_{6}^{-}\right]^{19+}($ calcd $\mathrm{m} / \mathrm{z}: 612.2)$, $574.3\left[{\mathrm{M}-20 \mathrm{PF}_{6}^{-}}^{-}\right]^{20+}(\operatorname{calcd} m / z: 574.3)$ and $540.1\left[{\mathrm{M}-21 \mathrm{PF}_{6}}^{-}\right]^{21+}(\operatorname{calcd} m / z: 540.1)$

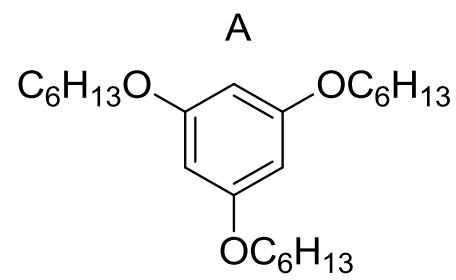


Compound 11. Benzene-1,3,5-triol (3.78 g, $30 \mathrm{mmol})$ and $\mathrm{K}_{2} \mathrm{CO}_{3}(24.8 \mathrm{~g} 180 \mathrm{mmol})$ were degassed solution three times. 1-bromohexane (20 g, $120 \mathrm{mmol})$ and DMF 150 $\mathrm{ml}$ were added. The solution was stirred at $85{ }^{\circ} \mathrm{C}$ for $3 \mathrm{~d}$ under $\mathrm{N}_{2}$. After that, it was cooled to room temperature. Solution was added $500 \mathrm{ml}$ water and extracted with $\mathrm{CH}_{2} \mathrm{Cl}_{2}$, and the combined organic phase was washed with brine and dried with anhydrous $\mathrm{Na}_{2} \mathrm{SO}_{4}$. The crude was purified by column chromatography on silica gel with DCM: hexane (1:4) as eluent to afford the product as a liquid $(7.5 \mathrm{~g}, 66 \%) .{ }^{1} \mathrm{H}$ NMR (400 MHz, $\left.\mathrm{CDCl}_{3}\right) \delta 6.09\left(\mathrm{~s}, 1 \mathrm{H}, \mathrm{Ph}-H^{\mathrm{A}}\right), 3.93(\mathrm{t}, J=6.6 \mathrm{~Hz}, 2 \mathrm{H}), 1.89-1.69$ $(\mathrm{m}, 2 \mathrm{H}), 1.52-1.41(\mathrm{~m}, 2 \mathrm{H}), 1.36(\mathrm{~m}, 4 \mathrm{H}), 0.94(\mathrm{t}, J=6.8 \mathrm{~Hz}, 3 \mathrm{H}) .{ }^{13} \mathrm{C}$ NMR $(100$ $\left.\mathrm{MHz}, \mathrm{CDCl}_{3}\right) \delta 160.98,93.79,68.01,31.60,29.24,25.75,22.62,14.04$.

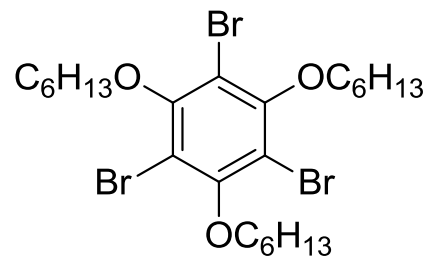

Compound 12. Compound $11(4.0 \mathrm{~g}, 10.5 \mathrm{mmol})$ was dissolved in $70 \mathrm{ml} \mathrm{CHCl}_{3}$. $\mathrm{FeCl}_{3}(170 \mathrm{mg}, 1.05 \mathrm{mmol})$ was added and solution was stirred at room temperature 10 min. A solution of $\mathrm{Br}_{2}(6.7 \mathrm{~g}, 42 \mathrm{mmol})$ in $15 \mathrm{ml} \mathrm{CHCl}{ }_{3}$ was slowly added. Solution was stirred at room temperature $1 \mathrm{~h}$ and refluxed overnight. It was cooled down and added water $40 \mathrm{ml}$ with $\mathrm{Na}_{2} \mathrm{~S}_{2} \mathrm{O}_{3} 4.0 \mathrm{~g}$. Solvent was removed and purified with column chromatography on silica gel with hexane as eluent to afford a white solid (5.5 g, $85 \%) .{ }^{1} \mathrm{H}$ NMR (400 MHz, $\left.\mathrm{CDCl}_{3}\right) \delta 4.00(\mathrm{t},, J=6.4 \mathrm{~Hz}, 2 \mathrm{H}), 1.96-$ $1.85(\mathrm{~m}, 2 \mathrm{H}), 1.64-1.49(\mathrm{~m}, 2 \mathrm{H}), 1.47-1.34(\mathrm{~m}, 4 \mathrm{H}), 0.94(\mathrm{t},, J=7.2 \mathrm{~Hz}, 3 \mathrm{H}) .{ }^{13} \mathrm{C}$ NMR $\left(100 \mathrm{MHz}, \mathrm{CDCl}_{3}\right) \delta 154.27,110.23,73.66,31.65,29.94,25.52,22.62,14.06$. 


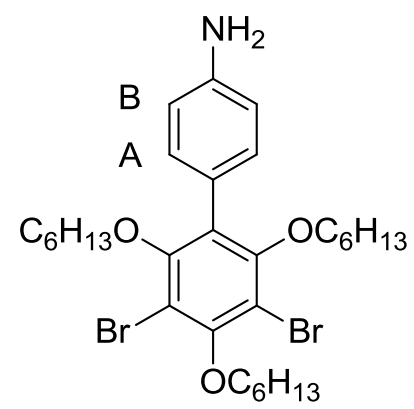

Compound 13. To a flask containing a degassed solution of compound $\mathbf{1 2}(6.0 \mathrm{~g}, 9.8$ mmol), 4-(4,4,5,5-tetramethyl-1,3,2-dioxaborolan-2-yl)aniline (700 mg, $3.2 \mathrm{mmol})$ in toluene $(60 \mathrm{~mL})$, an aqueous solution of $2 \mathrm{M} \mathrm{K}_{2} \mathrm{CO}_{3}(25 \mathrm{~mL})$ was added. $\mathrm{Pd}\left(\mathrm{PPh}_{3}\right)_{2} \mathrm{Cl}_{2}(140 \mathrm{mg}, 0.2 \mathrm{mmol})$ was then added under $\mathrm{N}_{2}$. The mixture was stirred at $75{ }^{\circ} \mathrm{C}$ overnight and then cooled to room temperature. The aqueous phase was extracted with $\mathrm{CH}_{2} \mathrm{Cl}_{2}$. The combined organic phase was washed with brine and dried with anhydrous $\mathrm{Na}_{2} \mathrm{SO}_{4}$. The crude was purified by column chromatography on silica gel with DCM: hexane (1:2) as eluent to afford the product as a white solid (1.1 g, $55 \%) .{ }^{1} \mathrm{H}$ NMR $\left(400 \mathrm{MHz}, \mathrm{CDCl}_{3}\right) \delta 7.24\left(\mathrm{~d}, J=8.5 \mathrm{~Hz}, 2 \mathrm{H}, \mathrm{Ph}-H^{\mathrm{A}}\right), 6.75(\mathrm{~d}, J=8.4$ $\left.\mathrm{Hz}, 2 \mathrm{H}, \mathrm{Ph}-H^{\mathrm{B}}\right), 4.05(\mathrm{t}, J=6.6 \mathrm{~Hz}, 2 \mathrm{H}), 3.48(\mathrm{t}, J=6.5 \mathrm{~Hz}, 4 \mathrm{H}), 1.97-1.86(\mathrm{~m}, 2 \mathrm{H})$, $1.62-1.52(\mathrm{~m}, 2 \mathrm{H}), 1.50-1.38(\mathrm{~m}, 8 \mathrm{H}), 1.32-1.10(\mathrm{~m}, 12 \mathrm{H}), 0.97-0.82(\mathrm{~m}, 9 \mathrm{H})$.

${ }^{13} \mathrm{C}$ NMR $\left(100 \mathrm{MHz}, \mathrm{CDCl}_{3}\right) \delta 154.82,153.37,145.43,131.63,128.08,123.23$, $114.63,109.90,73.48,73.15,31.70,31.45,30.02,29.77,25.59,25.42,22.64,22.55$, 14.09, 14.02. ESI-MS (m/z): Calcd. for $\left[\mathrm{C}_{30} \mathrm{H}_{45} \mathrm{Br}_{2} \mathrm{NO}_{3}+\mathrm{H}\right]^{+}$: 626.2. Found: 626.2. 


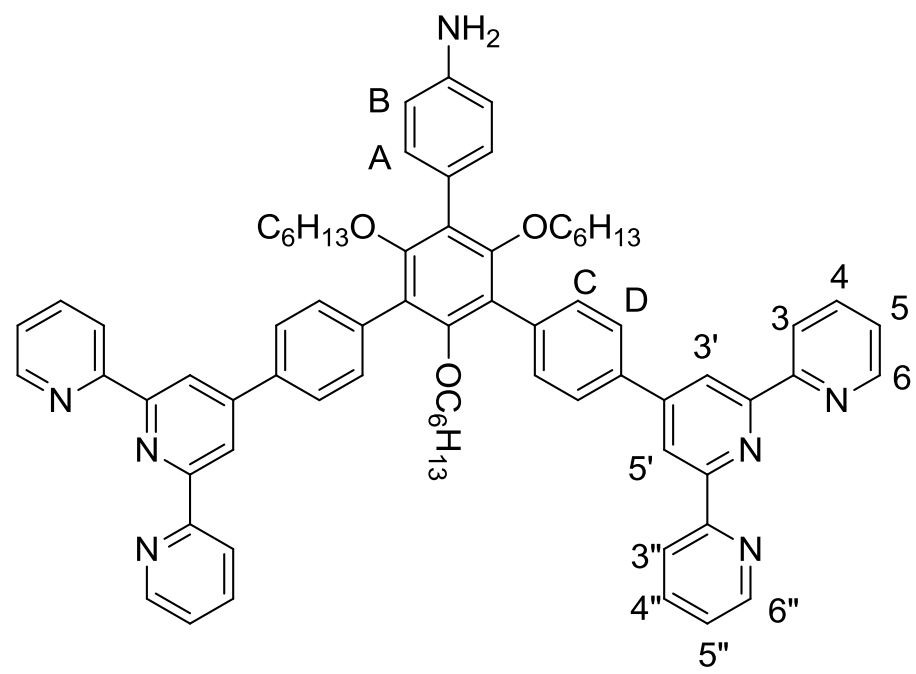

Compound 14. To a solution of compound $13(700 \mathrm{mg}, 1.1 \mathrm{mmol}), \mathrm{Pd}\left(\mathrm{PPh}_{3}\right)_{2} \mathrm{Cl}_{2}(80$ $\mathrm{mg}, 114 \mu \mathrm{mol})$ and 4'-(4-Boronatophenyl)[2,2':6',2"]terpyridine (1.58 g, $4.5 \mathrm{mmol})$ in toluene $(50 \mathrm{~mL})$ and tert-butyl alcohol $(8 \mathrm{ml})$ under $\mathrm{N}_{2}$, aqueous $1 \mathrm{M} \mathrm{K}_{2} \mathrm{CO}_{3}(20 \mathrm{~mL})$ was added. After refluxing for $48 \mathrm{~h}$, the mixture was cooled to room temperature. The aqueous layer was extracted with $\mathrm{CHCl}_{3}$ and the combined organic phase was washed with brine and dried over $\mathrm{Na}_{2} \mathrm{SO}_{4}$. After removal of solvent under vacuum, the residue was purified by column chromatography on silica gel with chloroform: ethanol (100:2) as eluent to afford a white solid in $82 \%$ yield. ${ }^{1} \mathrm{H}$ NMR $\left(400 \mathrm{MHz}, \mathrm{CDCl}_{3}\right) \delta 8.83(\mathrm{~s}$, $\left.4 \mathrm{H}, \mathrm{tpy}-H^{3^{\prime}, 5^{\prime}}\right), 8.76\left(\mathrm{ddd}, J=4.8,1.8,0.9 \mathrm{~Hz}, 4 \mathrm{H}, \mathrm{tpy}-H^{6,6^{\prime \prime}}\right), 8.71(\mathrm{dt}, J=8.0,1.1 \mathrm{~Hz}$, 4H, tpy- $\left.H^{3,3 "}\right), 8.02-7.95\left(\mathrm{~d}, J=4.4 \mathrm{~Hz}, 4 \mathrm{H}, \mathrm{Ph}-H^{\mathrm{D}}\right), 7.90(\mathrm{ddd}, J=7.9,7.5,1.8 \mathrm{~Hz}$, 4H, tpy- $\left.H^{4,4 "}\right), 7.74-7.69\left(\mathrm{~d}, J=4.4 \mathrm{~Hz}, 4 \mathrm{H}, \mathrm{Ph}-H^{\mathrm{C}}\right), 7.40-7.34\left(\mathrm{~m}, 6 \mathrm{H}, \mathrm{tpy}-H^{5,5 "}\right.$ and $\left.\mathrm{Ph}-H^{\mathrm{A}}\right), 6.80-6.74\left(\mathrm{~d}, J=4.4 \mathrm{~Hz}, 2 \mathrm{H}, \mathrm{Ph}-H^{\mathrm{B}}\right), 3.28(\mathrm{q}, J=6.6 \mathrm{~Hz}, 6 \mathrm{H}), 1.16-$ $1.00(\mathrm{~m}, 12 \mathrm{H}), 1.00-0.83(\mathrm{~m}, 12 \mathrm{H}), 0.75(\mathrm{t}, J=7.2 \mathrm{~Hz}, 6 \mathrm{H}), 0.66(\mathrm{t}, J=7.2 \mathrm{~Hz}, 3 \mathrm{H})$. ${ }^{13} \mathrm{C}$ NMR $\left(100 \mathrm{MHz}, \mathrm{CDCl}_{3}\right) \delta 156.35,155.87,155.66,154.71,150.41,149.10$, $145.07,136.90,136.65,136.12,132.15,132.05,131.62,128.55,128.43,126.50$, $123.78,121.39,118.89,114.59,73.57,73.26,31.42,31.38,30.90,29.68,25.35,25.34$ 
22.53, 22.50, 13.97,13.91. ESI-MS (m/z): Calcd. for $\left[\mathrm{C}_{72} \mathrm{H}_{73} \mathrm{~N}_{7} \mathrm{O}_{3}+\mathrm{H}\right]^{+}$: 1084.6. Found: 1084.6.

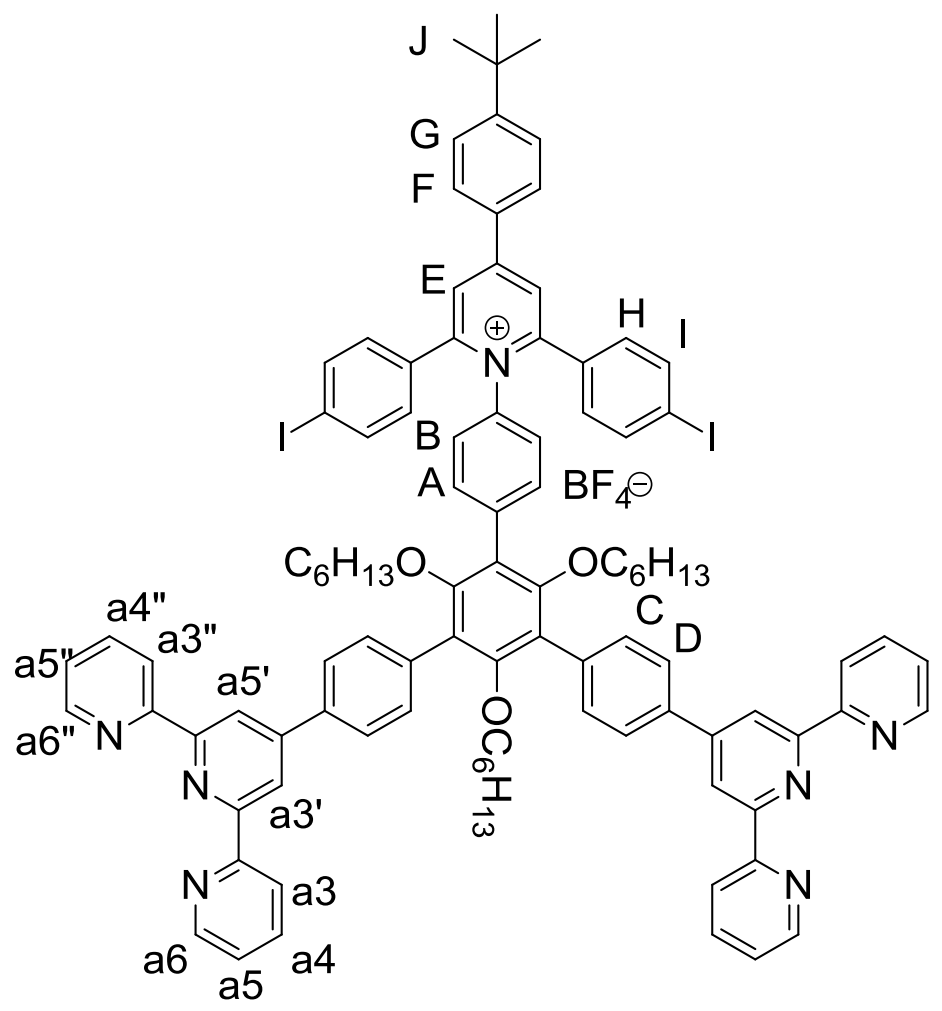

Compound 15. Anhydrous sodium acetate $(164 \mathrm{mg}, 2.0 \mathrm{mmol})$ and the pyrylium salt 1 (352 $\mathrm{mg}, 0.5 \mathrm{mmol})$ were added to a solution of $14(541 \mathrm{mg}, 0.5 \mathrm{mmol})$ in dry ethanol (15 mL). The mixture was reflux for $8 \mathrm{~h}$ and then cooled to room temperature. The precipitate was filtered and washed with water and diethyl ether to give the product as a white solid (708 mg, 80\%). ${ }^{1} \mathrm{H}$ NMR (400 MHz, DMSO- $\left.d_{6}\right) \delta 8.79\left(\mathrm{~s}, 4 \mathrm{H}\right.$, tpy- $\left.H^{3^{\prime}, 5^{\prime}}\right)$, $8.78-8.75\left(\mathrm{~m}, 4 \mathrm{H}, \mathrm{tpy}-H^{6,6 "}\right), 8.70\left(\mathrm{~d}, J=8.0 \mathrm{~Hz}, 4 \mathrm{H}, \mathrm{tpy}-H^{3,3^{\prime \prime}}\right), 8.61\left(\mathrm{~s}, 2 \mathrm{H}, \mathrm{Ph}-H^{\mathrm{E}}\right)$, $8.28\left(\mathrm{~d}, J=8.7 \mathrm{~Hz}, 2 \mathrm{H}, \mathrm{Ph}-H^{\mathrm{F}}\right), 8.10-8.01\left(\mathrm{~m}, 4 \mathrm{H}, \mathrm{tpy}-H^{4,4 "}\right), 7.97(\mathrm{~d}, J=8.4 \mathrm{~Hz}, 4 \mathrm{H}$, Ph- $\left.H^{\mathrm{D}}\right), 7.75\left(\mathrm{~d}, J=8.4 \mathrm{~Hz}, 4 \mathrm{H}, \mathrm{Ph}-H^{\mathrm{I}}\right), 7.70-7.64\left(\mathrm{~m}, 4 \mathrm{H}, \mathrm{Ph}-H^{\mathrm{G}}\right.$ and $\left.\mathrm{Ph}-H^{\mathrm{A}}\right), 7.62$ $\left(\mathrm{d}, J=8.3 \mathrm{~Hz}, 4 \mathrm{H}, \mathrm{Ph}-H^{\mathrm{C}}\right), 7.57-7.50\left(\mathrm{~m}, 4 \mathrm{H}, \mathrm{tpy}-H^{5,5^{\prime \prime}}\right), 7.44(\mathrm{~d}, J=8.6 \mathrm{~Hz}, 2 \mathrm{H}$, Ph- $\left.H^{\mathrm{B}}\right), 7.30\left(\mathrm{~d}, J=8.4 \mathrm{~Hz}, 4 \mathrm{H}, \mathrm{Ph}-H^{\mathrm{H}}\right), 3.33(\mathrm{~m}, 2 \mathrm{H}), 2.90(\mathrm{~m}, 4 \mathrm{H}), 1.36(\mathrm{~s}, 9 \mathrm{H}$, tert-butyl- $\left.H^{\mathrm{J}}\right), 1.04-0.94(\mathrm{~m}, 8 \mathrm{H}), 0.94-0.80(\mathrm{~m}, 8 \mathrm{H}), 0.80-0.70(\mathrm{~m}, 8 \mathrm{H}), 0.60(\mathrm{t}$, 
$J=7.2 \mathrm{~Hz}, 6 \mathrm{H}), 0.54(\mathrm{t}, J=7.2 \mathrm{~Hz}, 3 \mathrm{H}) .{ }^{13} \mathrm{C} \mathrm{NMR}\left(100 \mathrm{MHz}, \mathrm{DMSO}-d_{6}\right) \delta 156.59$, $156.31,156.06,155.94,155.84,155.63,155.41,149.91,149.76,138.08,137.83$, $137.50,136.98,136.86,135.98,133.06,132.24,132.07,131.52,130.91,129.17$, $128.56,127.04,126.58,126.05,125.67,124.88,124.32,121.39,118.43,97.98,73.54$ $73.32,35.34,31.43,31.28,31.10,29.78,29.48,25.51,25.12,22.54,22.30,14.14$, 13.96. ESI-MS (m/z): Calcd. for $\left[\mathrm{C}_{99} \mathrm{H}_{94} \mathrm{I}_{2} \mathrm{~N}_{7} \mathrm{O}_{3}\right]^{+}:$1682.5. Found: 1682.2.

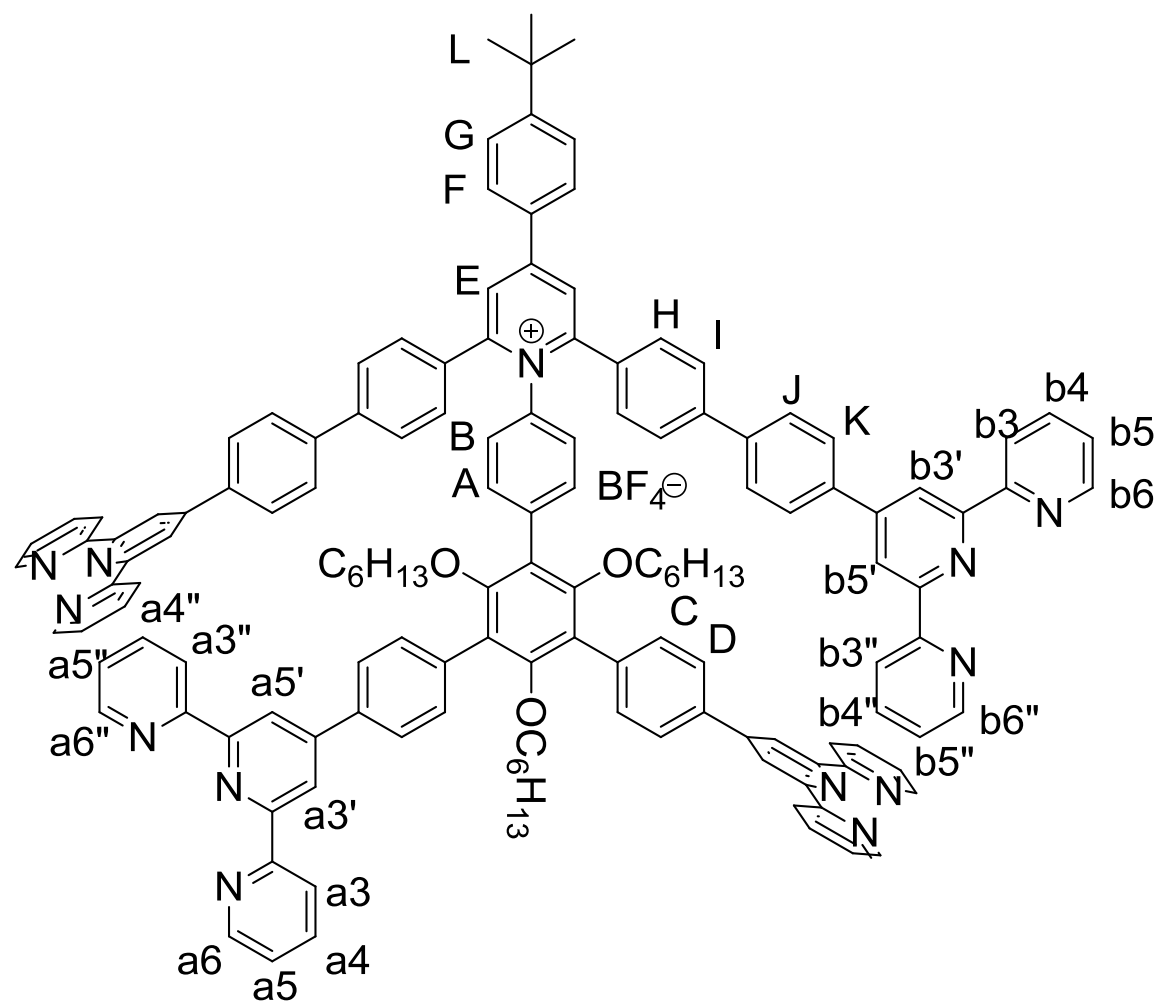

Ligand LC: To a solution of compound $15(177 \mathrm{mg}, 100 \mu \mathrm{mol}), \operatorname{Pd}\left(\mathrm{PPh}_{3}\right)_{2} \mathrm{Cl}_{2}(10.5$ $\mathrm{mg}, 15.0 \mu \mathrm{mol})$ and 4'-(4-Boronatophenyl)[2,2':6',2"]terpyridine (141 mg, $400 \mu \mathrm{mol})$ in DMSO $(25 \mathrm{~mL})$ under $\mathrm{N}_{2}$, aqueous $2 \mathrm{M} \mathrm{K}_{2} \mathrm{CO}_{3}(7 \mathrm{~mL})$ was added. The mixture was stirred at $80^{\circ} \mathrm{C}$ for $2 \mathrm{~d}$ and then cooled to room temperature. The mixture was added into $200 \mathrm{ml}$ water and was extracted with $\mathrm{CHCl}_{3}$, and the combined organic phase was washed with brine and dried over $\mathrm{Na}_{2} \mathrm{SO}_{4}$. After removal of solvent under vacuum, the residue was purified by column chromatography on $\mathrm{Al}_{2} \mathrm{O}_{3}$. The polarity 
of eluent was increased from chloroform/acetone $100 / 0$ to $0 / 100$, then a mixture of acetone/acetonitrile, $70 / 30$ to $0 / 100$ and finally a mixture of acetonitrile/water/ $\mathrm{NH}_{4} \mathrm{BF}_{4}$ to afford ligand $\mathbf{L C}$ as a white solid (66 \% yield). ${ }^{1} \mathrm{H}$ NMR $\left(400 \mathrm{MHz}, \mathrm{CDCl}_{3}\right) \delta 8.76\left(\mathrm{~s}, 4 \mathrm{H}\right.$, tpy- $\left.H^{\mathrm{a} 3^{\prime}, 5^{\prime}}\right), 8.75\left(\mathrm{~s}, 4 \mathrm{H}, \mathrm{tpy}-H^{\mathrm{b} 3^{\prime}, 5^{\prime}}\right), 8.69-8.63(\mathrm{~m}$, $16 \mathrm{H}$, tpy- $H^{\mathrm{a} 3,3 "}$, tpy- $H^{\mathrm{a} 6,6^{\prime \prime}}$, tpy- $H^{\mathrm{b} 3,3^{\prime \prime}}$ and tpy- $\left.H^{\mathrm{b} 6,6 "}\right), 8.23\left(\mathrm{~s}, 2 \mathrm{H}, \mathrm{Ph}-H^{\mathrm{E}}\right), 8.00-7.95$ $\left(\mathrm{m}, 6 \mathrm{H}, \mathrm{Ph}-H^{\mathrm{D}}\right.$ and $\left.\mathrm{Ph}-H^{\mathrm{F}}\right), 7.93\left(\mathrm{~d}, J=8.4 \mathrm{~Hz}, 4 \mathrm{H}, \mathrm{Ph}-H^{\mathrm{K}}\right), 7.90-7.83(\mathrm{~m}, 8 \mathrm{H}$, tpy- $H^{\mathrm{a} 4,4^{\prime \prime}}$ and tpy- $\left.H^{\mathrm{b} 4,4 "}\right), 7.79\left(\mathrm{~d}, J=8.4 \mathrm{~Hz}, 4 \mathrm{H}, \mathrm{Ph}-H^{\mathrm{I}}\right), 7.74(\mathrm{~d}, J=8.6 \mathrm{~Hz}, 2 \mathrm{H}$, $\left.\mathrm{Ph}-H^{\mathrm{A}}\right), 7.69-7.63\left(\mathrm{~m}, 8 \mathrm{H}, \mathrm{Ph}-H^{\mathrm{C}}\right.$ and $\left.\mathrm{Ph}-H^{\mathrm{H}}\right), 7.62\left(\mathrm{~d}, J=5.5 \mathrm{~Hz}, 2 \mathrm{H}, \mathrm{Ph}-H^{\mathrm{G}}\right), 7.60$ $-7.54\left(\mathrm{~m}, 6 \mathrm{H}, \mathrm{Ph}-H^{\mathrm{B}}\right.$ and $\left.\mathrm{Ph}-H^{\mathrm{J}}\right), 7.35-7.29\left(\mathrm{~m}, 8 \mathrm{H}, \mathrm{tpy}-H^{\mathrm{a} 5,5^{\prime \prime}}\right.$ and tpy- $\left.H^{\mathrm{b} 5,5^{\prime \prime}}\right), 3.23(\mathrm{t}$, $J=6.5 \mathrm{~Hz}, 2 \mathrm{H}), 2.95(\mathrm{t}, J=6.0 \mathrm{~Hz}, 4 \mathrm{H}), 1.41\left(\mathrm{~s}, 9 \mathrm{H}\right.$, tert-butyl- $\left.H^{\mathrm{L}}\right), 1.03(\mathrm{~m}, 6 \mathrm{H})$, $0.94-0.78(\mathrm{~m}, 10 \mathrm{H}), 0.78-0.70(\mathrm{~m}, 4 \mathrm{H}), 0.68-0.59(\mathrm{~m}, 7 \mathrm{H}), 0.55(\mathrm{t}, J=7.6 \mathrm{~Hz}$ $6 \mathrm{H}) .{ }^{13} \mathrm{C} \mathrm{NMR}\left(100 \mathrm{MHz}, \mathrm{CDCl}_{3}\right) \delta 156.87,156.65,156.36,156.24,156.08,156.05$, $155.93,155.81,155.18,150.11,149.34,149.07,149.06,141.84,139.73,138.28$, $137.76,137.09,137.02,136.84,136.80,135.46,132.52,131.84,131.49,131.40$, $130.69,128.40,128.16,127.97,127.95,127.42,126.86,126.63,126.19,126.16$, $123.80,123.71,123.69,121.30,121.26,118.86,118.71,73.68,73.32,35.15,31.40$, $31.32,31.11,29.74,29.53,25.40,25.23,22.59,22.44,13.95,13.86$. MALDI-TOF MS (m/z): Calcd. for $\left[\mathrm{C}_{105} \mathrm{H}_{82} \mathrm{~N}_{13} \mathrm{O}\right]^{+}$2045.0. Found: 2045.1 . 


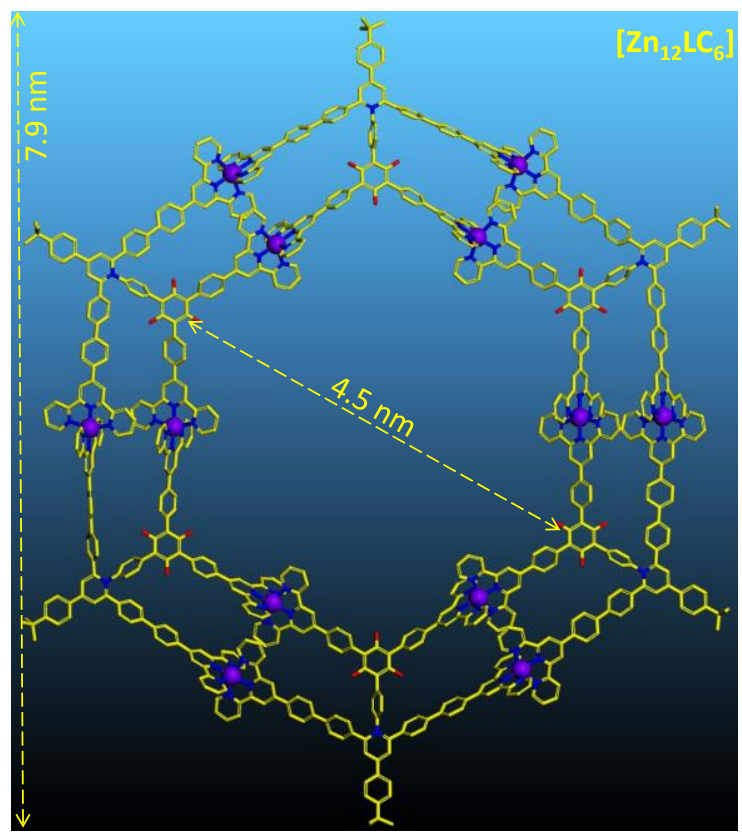

Complex $\left[\mathbf{Z n}_{12} \mathbf{L} \mathbf{C}_{6}\right]$ : To a solution of ligand $\mathbf{L C}(6.2 \mathrm{mg}, 2.9 \mu \mathrm{mol})$ in $\mathrm{CHCl}_{3}(1 \mathrm{~mL})$, a solution of $\mathrm{Zn}\left(\mathrm{NO}_{3}\right)_{2} \cdot 6 \mathrm{H}_{2} \mathrm{O}(1.7 \mathrm{mg}, 5.8 \mu \mathrm{mol})$ in $\mathrm{MeOH}(3 \mathrm{~mL})$ was added. The mixture was stirred at $50{ }^{\circ} \mathrm{C}$ for $8 \mathrm{~h}$ and then cooled to room temperature. Upon addition of $\mathrm{NH}_{4} \mathrm{PF}_{6}(150 \mathrm{mg})$, a precipitate was formed and washed with water to give a white product (yield: $91 \%) .{ }^{1} \mathrm{H}$ NMR $\left(400 \mathrm{MHz}, \mathrm{CD}_{3} \mathrm{CN}\right) \delta 8.98\left(\mathrm{~m}, 8 \mathrm{H}\right.$, tpy- $H^{\mathrm{a} 3^{\prime}, 5^{\prime}}$ and tpy- $\left.H^{\mathrm{b} 3^{\prime}, 5^{\prime}}\right), 8.73-8.49\left(\mathrm{~m}, 10 \mathrm{H}\right.$, tpy- $H^{\mathrm{a} 3,3^{\prime \prime}}$, tpy- $H^{\mathrm{b} 3,3^{\prime \prime}}$ and $\left.\mathrm{Ph}-H^{\mathrm{E}}\right), 8.33(\mathrm{~m}, 4 \mathrm{H}$, Ph- $\left.H^{\mathrm{D}}\right), 8.27\left(\mathrm{~d}, J=7.0 \mathrm{~Hz}, 4 \mathrm{H}, \mathrm{Ph}-H^{\mathrm{K}}\right), 8.21\left(\mathrm{~d}, J=7.6 \mathrm{~Hz}, 2 \mathrm{H}, \mathrm{Ph}-H^{\mathrm{F}}\right), 8.15(\mathrm{~d}, J=$ $\left.7.1 \mathrm{~Hz}, 4 \mathrm{H}, \mathrm{Ph}-H^{\mathrm{C}}\right), 8.02\left(\mathrm{~d}, J=7.5 \mathrm{~Hz}, 4 \mathrm{H}, \mathrm{Ph}-H^{\mathrm{I}}\right), 7.76\left(\mathrm{~m}, 28 \mathrm{H}, \mathrm{tpy}-H^{\mathrm{a} 44^{4}}\right.$, tрy- $H^{\mathrm{b} 4,4^{\prime \prime}}$, tрy- $H^{\mathrm{a} 6,6 "}$, tpy- $H^{\mathrm{b} 6,6^{\prime \prime}}, \mathrm{Ph}-H^{\mathrm{H}}, \mathrm{Ph}-H^{\mathrm{G}}, \mathrm{Ph}-H^{\mathrm{A}}$ and $\left.\mathrm{Ph}-H^{\mathrm{J}}\right), 7.59(\mathrm{~m}, 2 \mathrm{H}$, Ph- $\left.H^{\mathrm{B}}\right), 7.12-6.92\left(\mathrm{~m}, 8 \mathrm{H}, \mathrm{tpy}-H^{\mathrm{a} 5,5^{\prime \prime}}\right.$ and tpy- $\left.H^{\mathrm{b} 5,5 "}\right), 3.35(\mathrm{~s}, 2 \mathrm{H}), 3.12(\mathrm{~s}, 4 \mathrm{H}), 1.47$ $\left(\mathrm{s}, 9 \mathrm{H}\right.$, tert-butyl- $\left.H^{\mathrm{L}}\right), 1.10(\mathrm{~m}, 6 \mathrm{H}), 0.95(\mathrm{~m}, 10 \mathrm{H}), 0.71-0.50(\mathrm{~m}, 17 \mathrm{H}) .{ }^{13} \mathrm{C}$ NMR $\left(100 \mathrm{MHz}, \mathrm{CD}_{3} \mathrm{CN}\right) \delta 157.06,156.58,155.72,149.62,147.84,147.70,141.23,140.99$, $140.67,136.13,134.80,133.20,132.30,130.83,128.99,128.57,127.94,127.47$, $127.29,127.05,126.88,123.29,123.10,121.58,121.33,73.71,73.62,34.94,31.23$, 
$31.08,30.29,29.68,29.30,25.43,25.10,22.79,22.35,13.82,13.40$. ESI-MS $(\mathrm{m} / \mathrm{z})$ :

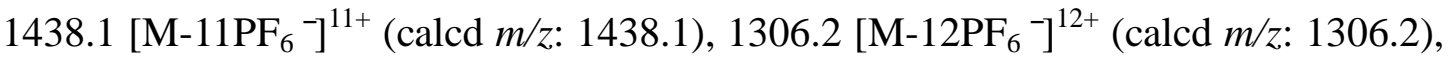
$1194.5\left[\mathrm{M}-13 \mathrm{PF}_{6}^{-}\right]^{13+}(\operatorname{calcd} m / z: 1194.5), 1098.8\left[{\mathrm{M}-14 \mathrm{PF}_{6}}^{-}\right]^{14+}(\operatorname{calcd} \mathrm{m} / z: 1098.8)$, $1015.9\left[{\mathrm{M}-15 \mathrm{PF}_{6}^{-}}^{-15+}(\right.$ calcd $m / z: 1015.9), 943.4\left[\mathrm{M}-16 \mathrm{PF}_{6}^{-}\right]^{16+}(\operatorname{calcd} \mathrm{m} / z: 943.4)$,

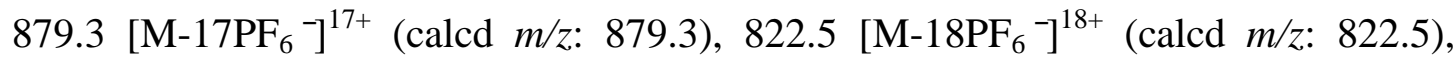
$771.5\left[{\mathrm{M}-19 \mathrm{PF}_{6}^{-}}^{-19+}(\right.$ calcd $m / z: 771.5), 725.7\left[{\mathrm{M}-20 \mathrm{PF}_{6}}^{-}\right]^{20+}($ calcd $m / z: 725.7)$, $684.3\left[\mathrm{M}-21 \mathrm{PF}_{6}^{-}\right]^{21+}($ calcd $\mathrm{m} / \mathrm{z}: 684.3), 646.6\left[\mathrm{M}^{-22 \mathrm{PF}_{6}}{ }^{-}\right]^{22+}($ calcd $\mathrm{m} / \mathrm{z}: 646.6)$, 612.1[M-23PF $\left.{ }_{6}^{-}\right]^{23+}(\operatorname{calcd} m / z: 612.1)$ and $580.6\left[{\mathrm{M}-24 \mathrm{PF}_{6}}^{-}\right]^{24+}(\operatorname{calcd} \mathrm{m} / \mathrm{z}: 580.6)$

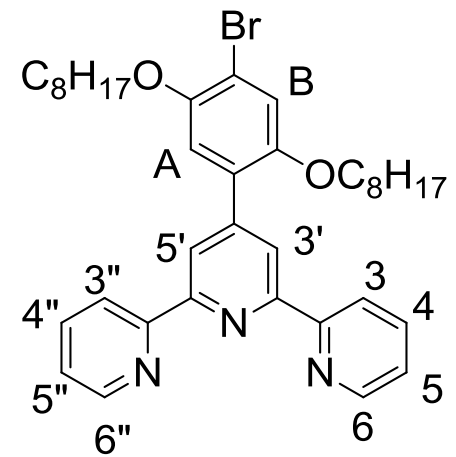

Compound 17. To a flask containing a degassed solution of 1,4-dibromo-2,5-bis(octyloxy)benzene ${ }^{8} \quad\left(\begin{array}{lllll}4.4 & \mathrm{~g}, & 9.0 & \mathrm{mmol}),\end{array}\right.$ 4'-(4-Boronatophenyl)[2,2':6',2"]terpyridine $(1.08 \mathrm{~g}, 3.0 \mathrm{mmol})$ in toluene $(60 \mathrm{~mL})$, an aqueous solution of $2 \mathrm{M} \mathrm{K}_{2} \mathrm{CO}_{3}(25 \mathrm{~mL})$ was added. $\mathrm{Pd}\left(\mathrm{PPh}_{3}\right)_{2} \mathrm{Cl}_{2}(105 \mathrm{mg}, 0.15$ mmol) was then added under $\mathrm{N}_{2}$. The mixture was stirred at $80^{\circ} \mathrm{C}$ for $20 \mathrm{~h}$ and then cooled to room temperature. The aqueous phase was extracted with $\mathrm{CHCl}_{3}$. The combined organic phase was washed with brine and dried with anhydrous $\mathrm{Na}_{2} \mathrm{SO}_{4}$. After removal of volatile under vacuum, the crude was purified by column chromatography on silica gel with chloroform: ethanol (200:1) as eluent to afford the product as a white solid $(1.08 \mathrm{~g}, 56 \%) .{ }^{1} \mathrm{H} \mathrm{NMR}\left(400 \mathrm{MHz}, \mathrm{CDCl}_{3}\right) \delta 8.72(\mathrm{ddd}, J=$ 
$4.8,1.8,0.9 \mathrm{~Hz}, 2 \mathrm{H}$, tpy- $\left.H^{6,6^{\prime \prime}}\right), 8.71-8.67\left(\mathrm{~m}, 4 \mathrm{H}\right.$, tpy- $H^{3,3^{\prime \prime}}$ and tpy- $\left.H^{3^{\prime}, 5^{\prime}}\right), 7.92-$ $7.86\left(\mathrm{~m}, 2 \mathrm{H}, \operatorname{tpy}-H^{4,4 "}\right), 7.35\left(\mathrm{ddd}, J=7.5,4.8,1.2 \mathrm{~Hz}, 2 \mathrm{H}, \mathrm{tpy}-H^{5,5 "}\right), 7.23(\mathrm{~s}, 1 \mathrm{H}$, Ph- $\left.H^{\mathrm{A}}\right), 7.13\left(\mathrm{~d}, J=4.7 \mathrm{~Hz}, 1 \mathrm{H}, \mathrm{Ph}-H^{\mathrm{B}}\right), 4.07(\mathrm{t}, J=6.5 \mathrm{~Hz}, 2 \mathrm{H}), 3.95(\mathrm{t}, J=6.3 \mathrm{~Hz}$, $2 \mathrm{H}), 1.91-1.80(\mathrm{~m}, 2 \mathrm{H}), 1.74-1.65(\mathrm{~m}, 2 \mathrm{H}), 1.57-1.48(\mathrm{~m}, 2 \mathrm{H}), 1.43-1.27(\mathrm{~m}$, 10H), $1.25-1.05(\mathrm{~m}, 8 \mathrm{H}), 0.91(\mathrm{t}, J=7.2 \mathrm{~Hz}, 3 \mathrm{H}), 0.82(\mathrm{t}, J=7.1 \mathrm{~Hz}, 3 \mathrm{H}) .{ }^{13} \mathrm{C} \mathrm{NMR}$ $\left(100 \mathrm{MHz}, \mathrm{CDCl}_{3}\right) \delta 156.37,155.21,150.75,150.00,149.07,147.73,136.82,128.49$, 123.66, 121.66, 121.28, 118.23, 116.03, 113.08, 70.55, 69.70, 31.82, 31.76, 29.34, 29.33, 29.24, 29.21, 29.05, 26.10, 26.02, 22.67, 22.58, 14.11, 14.06. ESI-MS (m/z): Calcd. for $\left[\mathrm{C}_{37} \mathrm{H}_{46} \mathrm{BrN}_{3} \mathrm{O}_{2}+\mathrm{H}\right]^{+}$: 644.3. Found: 644.3.

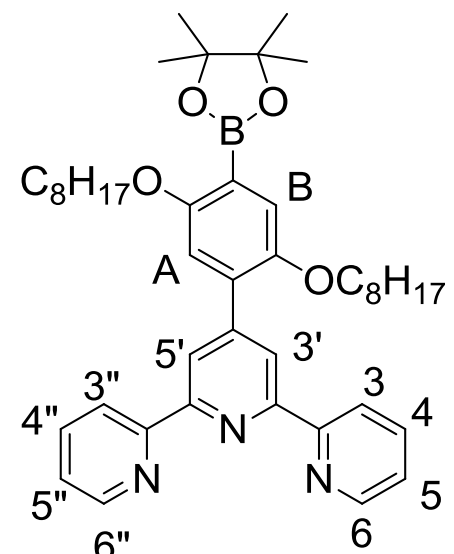

Compound 18. A mixture of compound $\mathbf{1 7}(1.28 \mathrm{~g}, 2.0 \mathrm{mmol})$, Bis(pinacolato)diboron (508 mg, $2.0 \mathrm{mmol}), \mathrm{Pd}(\mathrm{dppf}) \mathrm{Cl}_{2}(65 \mathrm{mg}, 0.08 \mathrm{mmol})$ and potassium acetate $(980 \mathrm{mg}, 10 \mathrm{mmol})$ was degassed three times. After that, $30 \mathrm{ml}$ anhydrous DMSO was added and then the mixture was stirred at $80{ }^{\circ} \mathrm{C}$ for $16 \mathrm{~h}$ and then cooled to room temperature. The aqueous phase was extracted with $\mathrm{CHCl}_{3}$. The combined organic phase was washed with water. After removal of volatile under vacuum, the crude was directly used for next step. 


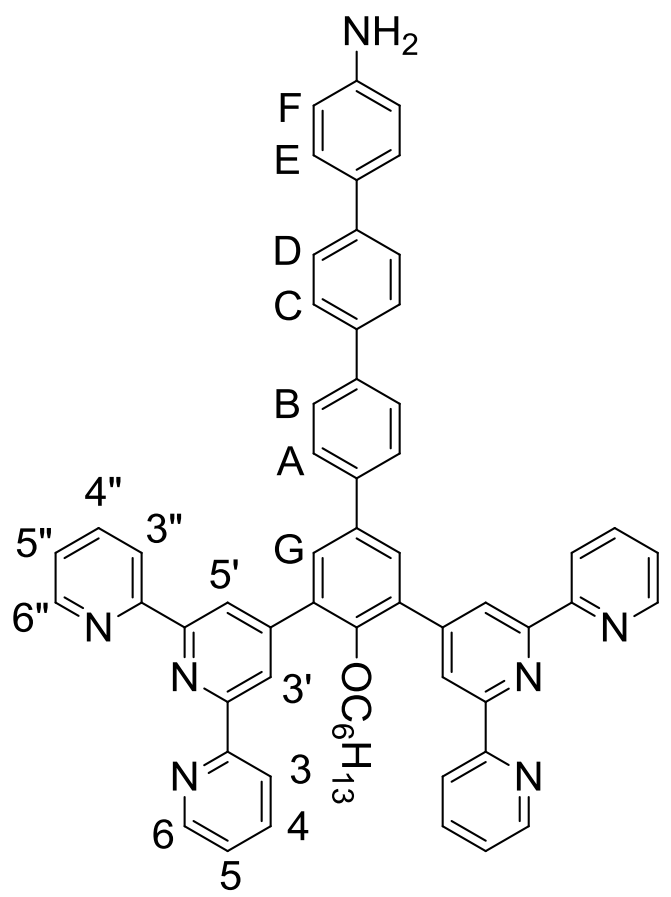

Compound 20. To a solution of compound $\mathbf{1 9}^{9}$ (668 mg, $\left.\left.1.8 \mathrm{mmol}\right), \mathrm{Pd}_{(\mathrm{PPh}}\right)_{2} \mathrm{Cl}_{2}$ (100 mg, $143 \mu \mathrm{mol})$ and compound $6(1.08 \mathrm{~g}, 1.5 \mathrm{mmol})$ in toluene (40 mL) under $\mathrm{N}_{2}$, aqueous $1.5 \mathrm{M} \mathrm{K}_{2} \mathrm{CO}_{3}(20 \mathrm{~mL})$ was added. It was heated at $75{ }^{\circ} \mathrm{C}$ overnight. The mixture was cooled to room temperature. The aqueous layer was extracted with $\mathrm{CHCl}_{3}$, and the combined organic phase was washed with brine and dried over $\mathrm{Na}_{2} \mathrm{SO}_{4}$. After removal of solvent under vacuum, the residue was purified by column chromatography on silica gel with chloroform: ethanol (100:3) as eluent to afford a white solid in $75 \%$ yield. ${ }^{1} \mathrm{H}$ NMR $\left(400 \mathrm{MHz}, \mathrm{CDCl}_{3}\right) \delta 8.90\left(\mathrm{~s}, 4 \mathrm{H}\right.$, tpy- $\left.H^{3^{\prime}, 5^{\prime}}\right), 8.79$ $\left(\mathrm{d}, J=4.0 \mathrm{~Hz}, 4 \mathrm{H}, \mathrm{tpy}-H^{6,6 "}\right), 8.74\left(\mathrm{~d}, J=8.0 \mathrm{~Hz}, 4 \mathrm{H}, \mathrm{tpy}-H^{3,3^{\prime \prime}}\right), 7.97-7.88(\mathrm{~m}, 6 \mathrm{H}$, tpy- $H^{4,4 "}$ and $\left.\mathrm{Ph}-H^{\mathrm{G}}\right), 7.83\left(\mathrm{~d}, J=8.4 \mathrm{~Hz}, 2 \mathrm{H}, \mathrm{Ph}-H^{\mathrm{A}}\right), 7.76\left(\mathrm{~d}, J=8.5 \mathrm{~Hz}, 2 \mathrm{H}, \mathrm{Ph}-H^{\mathrm{B}}\right)$, $7.72\left(\mathrm{~d}, J=8.5 \mathrm{~Hz}, 2 \mathrm{H}, \mathrm{Ph}-H^{\mathrm{C}}\right), 7.66\left(\mathrm{~d}, J=8.5 \mathrm{~Hz}, 2 \mathrm{H}, \mathrm{Ph}-H^{\mathrm{D}}\right), 7.50(\mathrm{~d}, J=8.5 \mathrm{~Hz}$, $\left.2 \mathrm{H}, \mathrm{Ph}-H^{\mathrm{E}}\right), 7.42-7.37\left(\mathrm{~m}, 4 \mathrm{H}, \mathrm{tpy}-H^{5,5 "}\right), 6.81\left(\mathrm{~d}, J=8.4 \mathrm{~Hz}, 2 \mathrm{H}, \mathrm{Ph}-H^{\mathrm{F}}\right), 3.45(\mathrm{t}, J$ $=6.1 \mathrm{~Hz}, 2 \mathrm{H}), 1.25(\mathrm{~m}, 2 \mathrm{H}), 0.96(\mathrm{~m}, 2 \mathrm{H}), 0.78(\mathrm{~m}, 4 \mathrm{H}), 0.53(\mathrm{t}, J=6.7 \mathrm{~Hz}, 3 \mathrm{H}) .{ }^{13} \mathrm{C}$ NMR $\left(100 \mathrm{MHz}, \mathrm{CDCl}_{3}\right) \delta 156.28,155.43,154.28,149.16,148.59,145.92,140.20$, 
$139.93,139.15,138.79,138.53,137.08,136.90,136.00,134.35,131.08,131.07$, 129.76, 127.94, 127.62, 127.32, 126.74, 123.77, 121.86, 121.35, 115.45, 74.35, 31.36, 29.81, 25.48, 22.16, 13.80. ESI-MS (m/z): Calcd. for $\left[\mathrm{C}_{60} \mathrm{H}_{49} \mathrm{~N}_{7} \mathrm{O}+\mathrm{H}\right]^{+}:$: 884.4. Found: 884.4

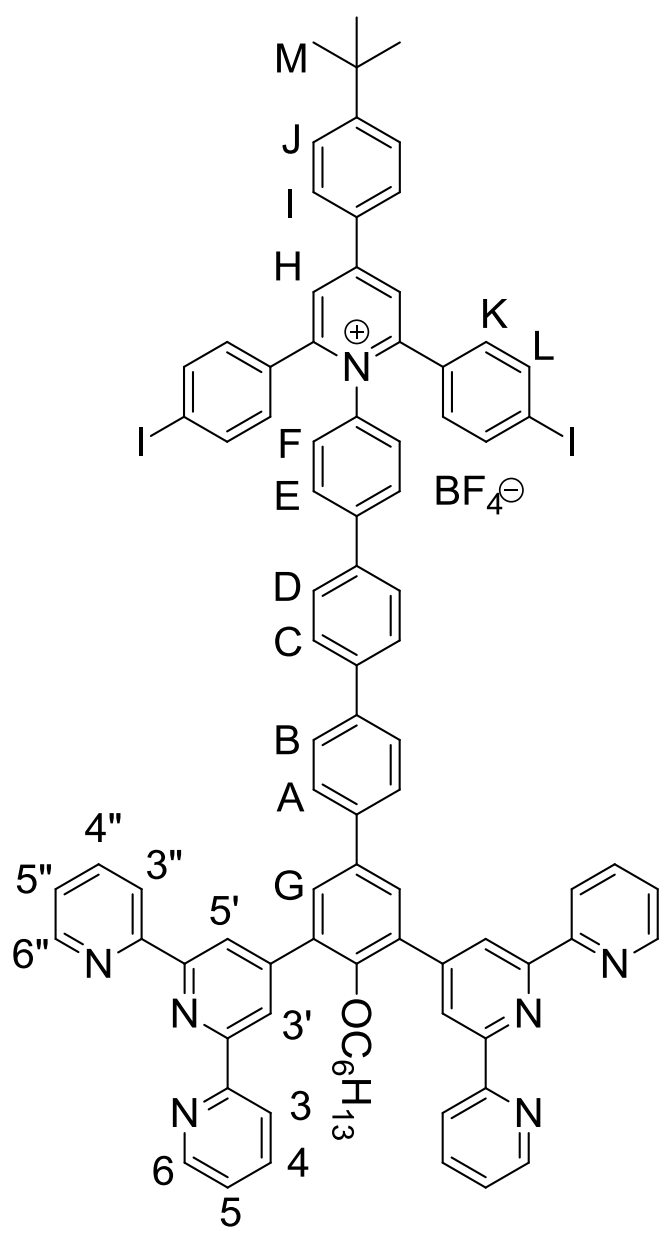

Compound 21. Anhydrous sodium acetate $(328 \mathrm{mg}, 4.0 \mathrm{mmol})$ and the pyrylium salt 1 (704 mg, $1.0 \mathrm{mmol}$ ) were added to a solution of $\mathbf{2 0}(706 \mathrm{mg}, 0.8 \mathrm{mmol})$ in dry ethanol $(25 \mathrm{~mL})$. The mixture was reflux for $16 \mathrm{~h}$ and then cooled to room temperature. The precipitate was filtered and washed with water and diethyl ether to give the product as a white solid $(891 \mathrm{mg}, 71 \%) .{ }^{1} \mathrm{H}$ NMR $\left(400 \mathrm{MHz}, \mathrm{CDCl}_{3}\right) \delta 8.89\left(\mathrm{~s}, 4 \mathrm{H}, \mathrm{tpy}-H^{3^{\prime}, 5^{\prime}}\right)$, $8.78\left(\mathrm{ddd}, J=4.8,1.8,0.9 \mathrm{~Hz}, 4 \mathrm{H}\right.$, tpy- $\left.H^{6,6 "}\right), 8.73\left(\mathrm{dt}, J=8.0,1.1 \mathrm{~Hz}, 4 \mathrm{H}\right.$, tpy- $\left.H^{3,3 "}\right)$, $8.10\left(\mathrm{~s}, 2 \mathrm{H}, \mathrm{Ph}-H^{\mathrm{H}}\right), 7.89-7.95\left(\mathrm{~m}, 6 \mathrm{H}\right.$, tpy- $H^{4,4 "}$ and $\left.\mathrm{Ph}-H^{\mathrm{G}}\right), 7.86(\mathrm{~d}, J=8.7 \mathrm{~Hz}, 2 \mathrm{H}$, 
$\left.\mathrm{Ph}-H^{\mathrm{I}}\right), 7.83\left(\mathrm{~d}, J=8.6 \mathrm{~Hz}, 2 \mathrm{H}, \mathrm{Ph}-H^{\mathrm{A}}\right), 7.71-7.77\left(\mathrm{~m}, 4 \mathrm{H}, \mathrm{Ph}-H^{\mathrm{B}}\right.$ and $\left.\mathrm{Ph}-H^{\mathrm{C}}\right), 7.70$ $-7.66\left(\mathrm{~m}, 4 \mathrm{H}, \mathrm{tpy}-H^{\mathrm{L}}\right), 7.64-7.60\left(\mathrm{~m}, 4 \mathrm{H}, \mathrm{Ph}-H^{\mathrm{J}}\right.$ and $\left.\mathrm{Ph}-H^{\mathrm{D}}\right), 7.52(\mathrm{~d}, J=8.8 \mathrm{~Hz}, 2 \mathrm{H}$, $\left.\mathrm{Ph}-H^{\mathrm{F}}\right), 7.43-7.36\left(\mathrm{~m}, 6 \mathrm{H}, \mathrm{tpy}-H^{5,5^{\prime \prime}}\right.$ and tpy- $\left.H^{\mathrm{E}}\right), 7.28-7.23\left(\mathrm{~m}, 4 \mathrm{H}, \mathrm{tpy}-H^{\mathrm{K}}\right), 3.45(\mathrm{t}$, $J=6.0 \mathrm{~Hz}, 2 \mathrm{H}), 1.49\left(\mathrm{~s}, 9 \mathrm{H}\right.$, tert-butyl- $\left.H^{\mathrm{M}}\right), 1.28-1.18(\mathrm{~m}, 2 \mathrm{H}), 0.95(\mathrm{~s}, 2 \mathrm{H}), 0.78$ (m, 4H), $0.53(\mathrm{t}, J=6.8 \mathrm{~Hz}, 3 \mathrm{H}) .{ }^{13} \mathrm{C}$ NMR (100 MHz, DMSO-d6) $\delta$ 157.87, 156.57, $156.34,155.83,155.52,154.42,149.22,148.48,142.36,140.98,139.44,139.19$, $139.07,137.82,136.80,134.44,132.36,131.29,131.23,129.80,129.75,128.87$, $128.31,127.74,127.67,127.47,127.44,126.92,126.46,126.34,126.08,123.75$, 121.76, 121.30, 99.99, 97.38, 74.35, 35.17, 31.36, 31.08, 29.79, 25.47, 22.16, 13.79 . ESI-MS (m/z): Calcd. for $\left[\mathrm{C}_{87} \mathrm{H}_{70} \mathrm{I}_{2} \mathrm{~N}_{7} \mathrm{O}\right]^{+}:$1482.4. Found: 1482.2.

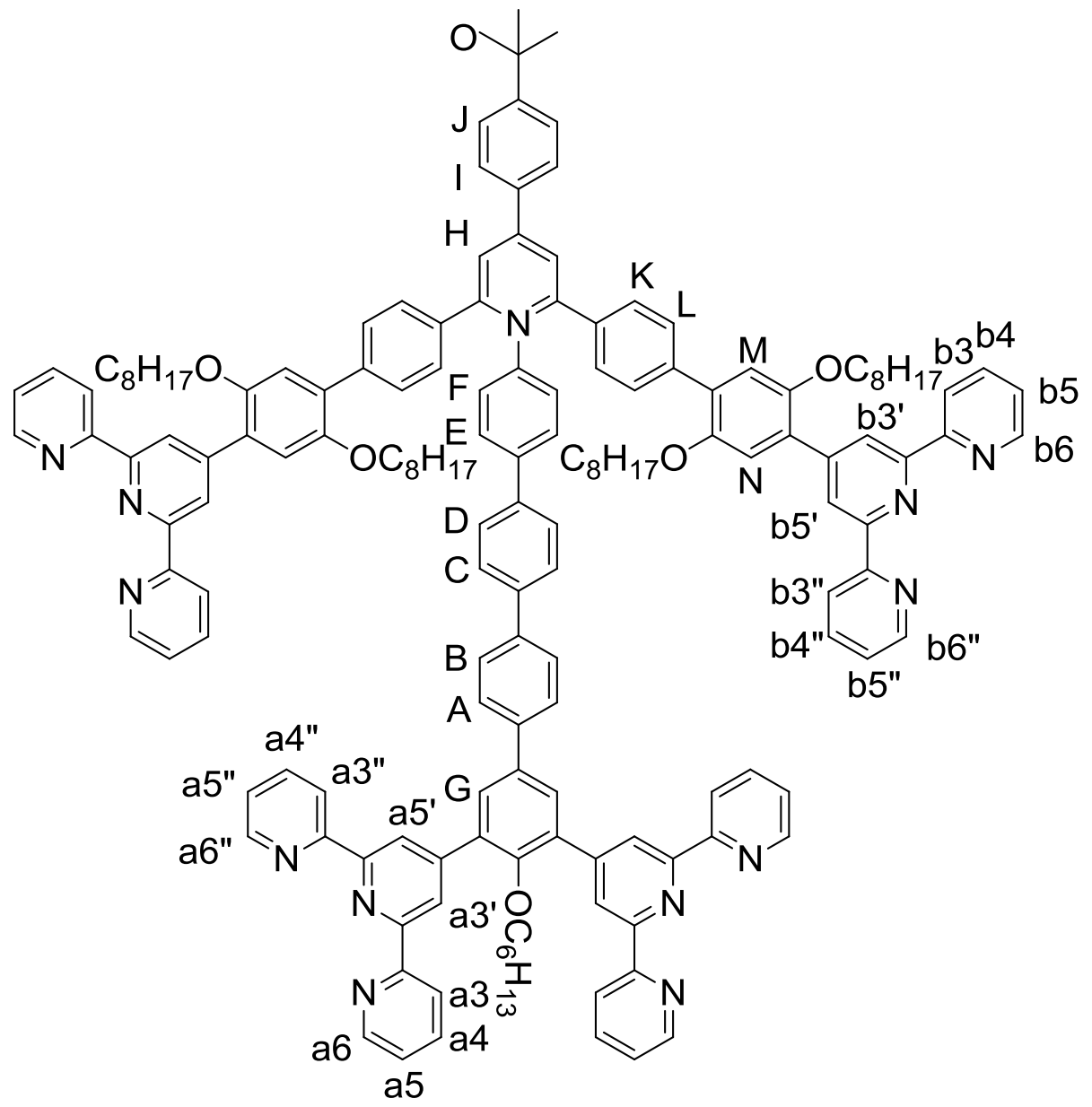

Ligand LD: To a solution of compound 21 (340 mg, $216 \mu \mathrm{mol}), \operatorname{Pd}\left(\mathrm{PPh}_{3}\right)_{2} \mathrm{Cl}_{2}(15 \mathrm{mg}$, 
$21.6 \mu \mathrm{mol})$ and compound $18(599 \mathrm{mg}, 867 \mu \mathrm{mol})$ in DMSO (40 mL) under $\mathrm{N}_{2}$, aqueous $2 \mathrm{M} \mathrm{K}_{2} \mathrm{CO}_{3}(10 \mathrm{~mL})$ was added. The mixture was stirred at $80{ }^{\circ} \mathrm{C}$ for $2 \mathrm{~d}$ and then cooled to room temperature. The mixture was extracted with $\mathrm{CHCl}_{3}$, and the combined organic phase was washed with brine and dried over $\mathrm{Na}_{2} \mathrm{SO}_{4}$. After removal of solvent under vacuum, the residue was purified by column chromatography on $\mathrm{Al}_{2} \mathrm{O}_{3}$. The polarity of eluent was increased from chloroform/acetone $100 / 0$ to $0 / 100$, then a mixture of acetone/acetonitrile, $70 / 30$ to $0 / 100$ and finally a mixture of acetonitrile/water/ $\mathrm{NH}_{4} \mathrm{BF}_{4}$ to afford $\mathrm{LD}$ as a white solid (61\% yield). ${ }^{1} \mathrm{H}$ NMR (400 $\left.\mathrm{MHz}, \mathrm{CDCl}_{3}\right) \delta 8.89-8.86\left(\mathrm{~s}, 4 \mathrm{H}, \mathrm{tpy}-H^{\mathrm{a} 3^{\prime}, 5^{\prime}}\right), 8.76(\mathrm{ddd}, J=4.8,1.8,0.9 \mathrm{~Hz}, 4 \mathrm{H}$, tpy- $\left.H^{\mathrm{a} 6,6^{\prime \prime}}\right), 8.72\left(\mathrm{dd}, J=5.5,2.3 \mathrm{~Hz}, 12 \mathrm{H}, \mathrm{tpy}-H^{\mathrm{a} 3,3^{\prime \prime}}, \mathrm{tpy}-H^{\mathrm{b} 3^{\prime}, 5^{\prime}}\right.$ and tpy- $\left.H^{\mathrm{b} 6,6^{\prime \prime}}\right), 8.68(\mathrm{~d}$, $\left.J=8.0 \mathrm{~Hz}, 4 \mathrm{H}, \mathrm{tpy}-H^{\mathrm{b} 3,3^{\prime \prime}}\right), 8.29\left(\mathrm{~s}, 2 \mathrm{H}, \mathrm{Ph}-H^{\mathrm{H}}\right), 8.01\left(\mathrm{~d}, J=8.8 \mathrm{~Hz}, 2 \mathrm{H}, \mathrm{Ph}-H^{\mathrm{I}}\right), 7.94$ $-7.85\left(\mathrm{~m}, 14 \mathrm{H}\right.$, tpy- $H^{\mathrm{a} 4,4 "}$, tpy- $H^{\mathrm{b} 44^{4}} \mathrm{Ph}-H^{\mathrm{F}}$ and $\left.\mathrm{Ph}-H^{\mathrm{G}}\right), 7.79(\mathrm{~d}, J=8.4 \mathrm{~Hz}, 2 \mathrm{H}$, $\left.\mathrm{Ph}-H^{\mathrm{A}}\right), 7.76\left(\mathrm{~d}, J=8.6 \mathrm{~Hz}, 4 \mathrm{H}, \mathrm{Ph}-H^{\mathrm{L}}\right), 7.71\left(\mathrm{~d}, J=8.1 \mathrm{~Hz}, 4 \mathrm{H}, \mathrm{Ph}-H^{\mathrm{B}}\right.$ and $\left.\mathrm{Ph}-H^{\mathrm{C}}\right)$, $7.68-7.63\left(\mathrm{~m}, 6 \mathrm{H}, \mathrm{Ph}-H^{\mathrm{K}}\right.$ and $\left.\mathrm{Ph}-H^{\mathrm{J}}\right), 7.59\left(\mathrm{~d}, J=8.6 \mathrm{~Hz}, 2 \mathrm{H}, \mathrm{Ph}-H^{\mathrm{D}}\right), 7.56-7.52$ $\left(\mathrm{m}, 2 \mathrm{H}, \mathrm{Ph}-H^{\mathrm{E}}\right), 7.39-7.31\left(\mathrm{~m}, 8 \mathrm{H}, \mathrm{tpy}-H^{\mathrm{a} 5,5^{\prime}}\right.$ and tpy- $\left.H^{\mathrm{b} 5,5 "}\right), 7.17\left(\mathrm{~s}, 2 \mathrm{H}, \mathrm{Ph}-H^{\mathrm{N}}\right)$, $6.97\left(\mathrm{~s}, 2 \mathrm{H}, \mathrm{Ph}-H^{\mathrm{M}}\right), 3.98(\mathrm{t}, J=6.2 \mathrm{~Hz}, 4 \mathrm{H}), 3.90(\mathrm{t}, J=6.7 \mathrm{~Hz}, 4 \mathrm{H}), 3.43(\mathrm{t}, J=6.0$ Hz, 2H), $1.69(\mathrm{~m}, 4 \mathrm{H}), 1.59(\mathrm{~m}, 4 \mathrm{H}), 1.42\left(\mathrm{~s}, 9 \mathrm{H}\right.$, tert-butyl- $\left.H^{\mathrm{O}}\right), 1.35(\mathrm{~m}, 4 \mathrm{H}), 1.31-$ $1.20(\mathrm{~m}, 22 \mathrm{H}), 1.20-1.12(\mathrm{~m}, 8 \mathrm{H}), 1.07(\mathrm{~m}, 8 \mathrm{H}), 0.97(\mathrm{~m}, 2 \mathrm{H}), 0.86(\mathrm{t}, J=6.9 \mathrm{~Hz}$, $6 \mathrm{H}), 0.82-0.75(\mathrm{~m}, 10 \mathrm{H}), 0.52(\mathrm{t}, J=6.7 \mathrm{~Hz}, 3 \mathrm{H}) .{ }^{13} \mathrm{C} \mathrm{NMR}\left(100 \mathrm{MHz}, \mathrm{CDCl}_{3}\right) \delta$ $156.98,156.45,156.41,156.30,155.46,155.15,154.36,150.79,150.26,149.17$, $149.06,148.51,148.02,141.94,140.59,140.32,139.30,139.20,138.50,137.71$, $136.88,136.84,134.38,131.70,131.37,130.44,129.75,129.74,129.60,129.53$, 
$129.10,128.31,127.68,127.51,127.42,127.35,127.08,126.85,125.81,123.74$ $123.60,121.84,121.79,121.30,115.98,115.70,100.00,74.35,69.79,69.71,35.18$, $31.83,31.76,31.35,31.12,29.79,29.38,29.36,29.28,29.20,29.18,29.05,26.17$, 25.90, 25.46, 22.66, 22.58, 22.15, 14.13, 14.06, 13.79. MALDI-TOF MS (m/z): Calcd. for $\left[\mathrm{C}_{105} \mathrm{H}_{82} \mathrm{~N}_{13} \mathrm{O}\right]^{+}$2357.3. Found: 2357.4.

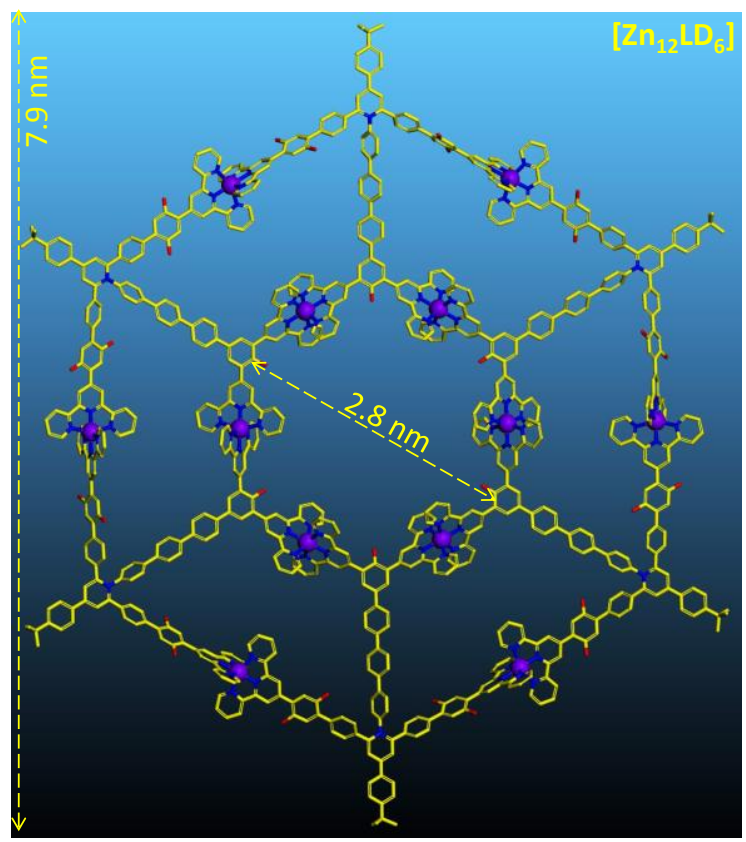

Complex $\left[\mathbf{Z n}_{12} \mathbf{L} \mathbf{D}_{6}\right]$ : To a solution of ligand $\mathbf{L D}(6.1 \mathrm{mg}, 2.5 \mu \mathrm{mol})$ in $\mathrm{CHCl}_{3}(1 \mathrm{~mL})$, a solution of $\mathrm{Zn}\left(\mathrm{NO}_{3}\right)_{2} \cdot 6 \mathrm{H}_{2} \mathrm{O}(1.5 \mathrm{mg}, 5.0 \mu \mathrm{mol})$ in $\mathrm{MeOH}(3 \mathrm{~mL})$ was added. The mixture was stirred at $50{ }^{\circ} \mathrm{C}$ for $10 \mathrm{~h}$ and then cooled to room temperature. Upon addition of $\mathrm{NH}_{4} \mathrm{PF}_{6}(130 \mathrm{mg})$, a precipitate was formed and washed with water to give a white product (yield: $88 \%) .{ }^{1} \mathrm{H}$ NMR $\left(400 \mathrm{MHz}, \mathrm{CD}_{3} \mathrm{CN}\right) \delta 9.35\left(\mathrm{~s}, 4 \mathrm{H}, \mathrm{tpy}-H^{\mathrm{a} 3^{\prime}, 5^{\prime}}\right)$, $9.05\left(\mathrm{~s}, 4 \mathrm{H}, \mathrm{tpy}-H^{\mathrm{b} 3^{\prime}, 5^{\prime}}\right), 8.84\left(\mathrm{~d}, J=8.2 \mathrm{~Hz}, 4 \mathrm{H}, \mathrm{tpy}-H^{\mathrm{a} 3,3^{\prime \prime}}\right), 8.67(\mathrm{~d}, J=7.8 \mathrm{~Hz}, 4 \mathrm{H}$, tpy- $\left.H^{\mathrm{b} 3,3 "}\right), 8.61\left(\mathrm{~s}, 2 \mathrm{H}, \mathrm{Ph}-H^{\mathrm{H}}\right), 8.52\left(\mathrm{~s}, 2 \mathrm{H}, \mathrm{Ph}-H^{\mathrm{G}}\right), 8.21\left(\mathrm{~m}, 12 \mathrm{H}\right.$, tpy- $H^{\mathrm{a} 4,4^{\prime \prime}}$, tpy- $H^{\mathrm{b} 4,4^{\prime \prime}}, \mathrm{Ph}-H^{\mathrm{I}}$ and $\left.\mathrm{Ph}-H^{\mathrm{F}}\right), 8.02\left(\mathrm{~d}, J=7.8 \mathrm{~Hz}, 2 \mathrm{H}, \mathrm{Ph}-H^{\mathrm{J}}\right), 7.96\left(\mathrm{~m}, 6 \mathrm{H}, \mathrm{tpy}-H^{\mathrm{a} 6,6 "}\right.$ and $\left.\mathrm{Ph}-H^{\mathrm{A}}\right), 7.90\left(\mathrm{~d}, J=5.1 \mathrm{~Hz}, 4 \mathrm{H}, \mathrm{tpy}-H^{\mathrm{b} 6,6^{\prime \prime}}\right), 7.82\left(\mathrm{~d}, J=6.7 \mathrm{~Hz}, 8 \mathrm{H}, \mathrm{Ph}-H^{\mathrm{L}}, \mathrm{Ph}-H^{\mathrm{E}}\right.$ 
and $\left.\mathrm{Ph}-H^{\mathrm{B}}\right), 7.73\left(\mathrm{~d}, J=8.3 \mathrm{~Hz}, 2 \mathrm{H}, \mathrm{Ph}-H^{\mathrm{C}}\right), 7.65\left(\mathrm{~d}, J=7.5 \mathrm{~Hz}, 4 \mathrm{H}, \mathrm{Ph}-H^{\mathrm{K}}\right), 7.55(\mathrm{~m}$, $4 \mathrm{H}, \mathrm{Ph}-H^{\mathrm{D}}$ and $\left.\mathrm{Ph}-H^{\mathrm{N}}\right), 7.51-7.41\left(\mathrm{~m}, 8 \mathrm{H}, \mathrm{tpy}-H^{\mathrm{a}, 55^{\prime \prime}}\right.$ and tpy- $\left.H^{\mathrm{b} 5,5^{\prime \prime}}\right), 7.30(\mathrm{~s}, 2 \mathrm{H}$, Ph- $\left.H^{\mathrm{M}}\right), 4.26(\mathrm{t}, J=6.0 \mathrm{~Hz}, 4 \mathrm{H}), 4.12(\mathrm{t}, J=6.3 \mathrm{~Hz}, 4 \mathrm{H}), 3.74(\mathrm{~s}, 2 \mathrm{H}), 1.89(\mathrm{~m}, 4 \mathrm{H})$, $1.65(\mathrm{~m}, 4 \mathrm{H}), 1.49(\mathrm{~s}, 15 \mathrm{H}), 1.30(\mathrm{~m}, 30 \mathrm{H}), 1.08-0.95(\mathrm{~m}, 20 \mathrm{H}), 0.90(\mathrm{t}, J=6.5 \mathrm{~Hz}$, $6 \mathrm{H}), 0.62(\mathrm{t}, J=6.8 \mathrm{~Hz}, 6 \mathrm{H}), 0.28(\mathrm{t}, J=7.3 \mathrm{~Hz}, 3 \mathrm{H}) .{ }^{13} \mathrm{C} \mathrm{NMR}\left(100 \mathrm{MHz}, \mathrm{CD}_{3} \mathrm{CN}\right)$ $\delta 157.00,156.60,154.76,154.05,153.52,150.84,150.59,149.45,149.00,147.98$, $141.47,141.32,140.08,139.79,139.65,138.42,138.26,137.65,132.88,132.76$, $132.55,132.22,130.98,129.68,129.62,129.36,129.29,128.56,127.89,127.59$, $127.52,127.05,125.90,124.52,124.17,123.26,123.07,116.06,115.69,69.60,69.57$, $34.95,31.67,31.46,31.26,30.31,29.32,29.15,29.13,28.96,28.92,26.40,26.11$, 25.79, 22.47, 22.20, 21.97, 13.51, 13.24, 12.88. ESI-MS $(\mathrm{m} / \mathrm{z}): 1608.5\left[{\left.\mathrm{M}-11 \mathrm{PF}_{6}{ }^{-}\right]^{11+}}^{1+}\right.$ $($ calcd $m / z: 1608.5), 1462.4\left[\mathrm{M}-12 \mathrm{PF}_{6}^{-}\right]^{12+}(\operatorname{calcd} m / z: 1462.4), 1338.8\left[\mathrm{M}-13 \mathrm{PF}_{6}^{-}\right]^{13+}$ (calcd $m / z: 1338.8), 1232.8\left[{\mathrm{M}-14 \mathrm{PF}_{6}^{-}}^{-14+}(\operatorname{calcd} m / z: 1232.8), 1140.9\left[\mathrm{M}-15 \mathrm{PF}_{6}^{-}\right]^{15+}\right.$ (calcd $m / z: 1140.9), 1060.5\left[{\mathrm{M}-16 \mathrm{PF}_{6}}^{-}\right]^{16+}(\operatorname{calcd} m / z: 1060.5), 989.7\left[\mathrm{M}-17 \mathrm{PF}_{6}{ }^{-}\right]^{17+}$ (calcd $m / z: 989.7), 926.6\left[\mathrm{M}-18 \mathrm{PF}_{6}^{-}\right]^{18+}($ calcd $m / z: 926.6), 870.2\left[\mathrm{M}-19 \mathrm{PF}_{6}^{-}\right]^{19+}$ (calcd $m / z: 870.2), 819.5\left[{\mathrm{M}-20 \mathrm{PF}_{6}}^{-}\right]^{20+}($ calcd $m / z: 819.5)$ and $773.5\left[\mathrm{M}-21 \mathrm{PF}_{6}{ }^{-}\right]^{21+}$ $($ calcd $m / z: 773.5)$ 
4. ESI mass spectra data of complex $\left[Z n_{12} L A_{6}\right],\left[Z_{12} L B_{6}\right],\left[Z_{12} L C_{6}\right]$ and $\left[\mathrm{Zn}_{12} \mathrm{LD}_{6}\right]\left(\mathrm{PF}_{6}{ }^{-}\right.$as counterion $)$.
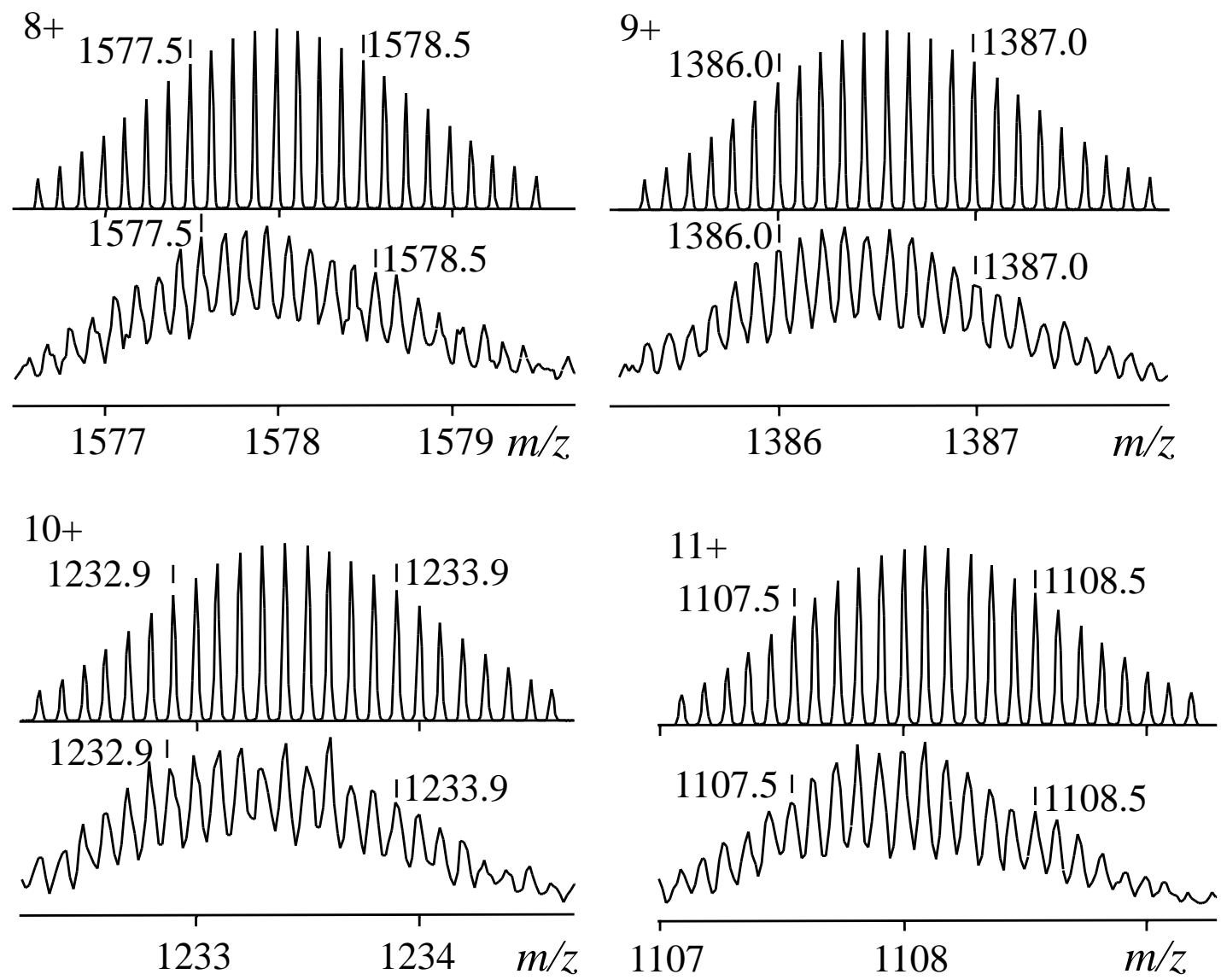

$12+$
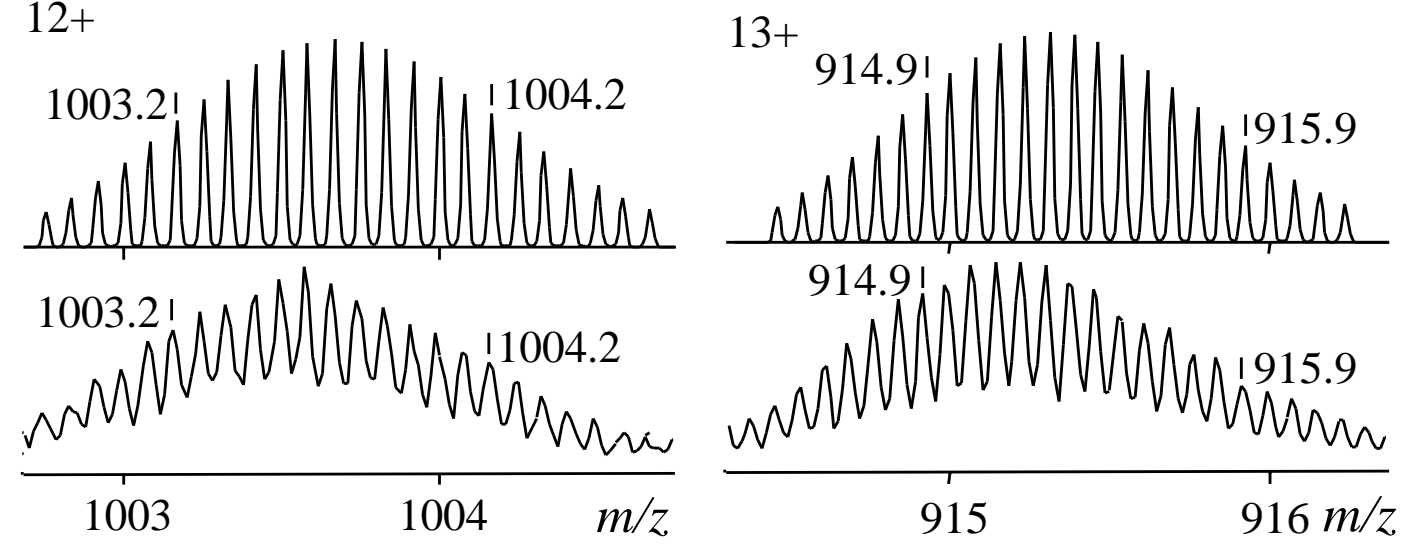

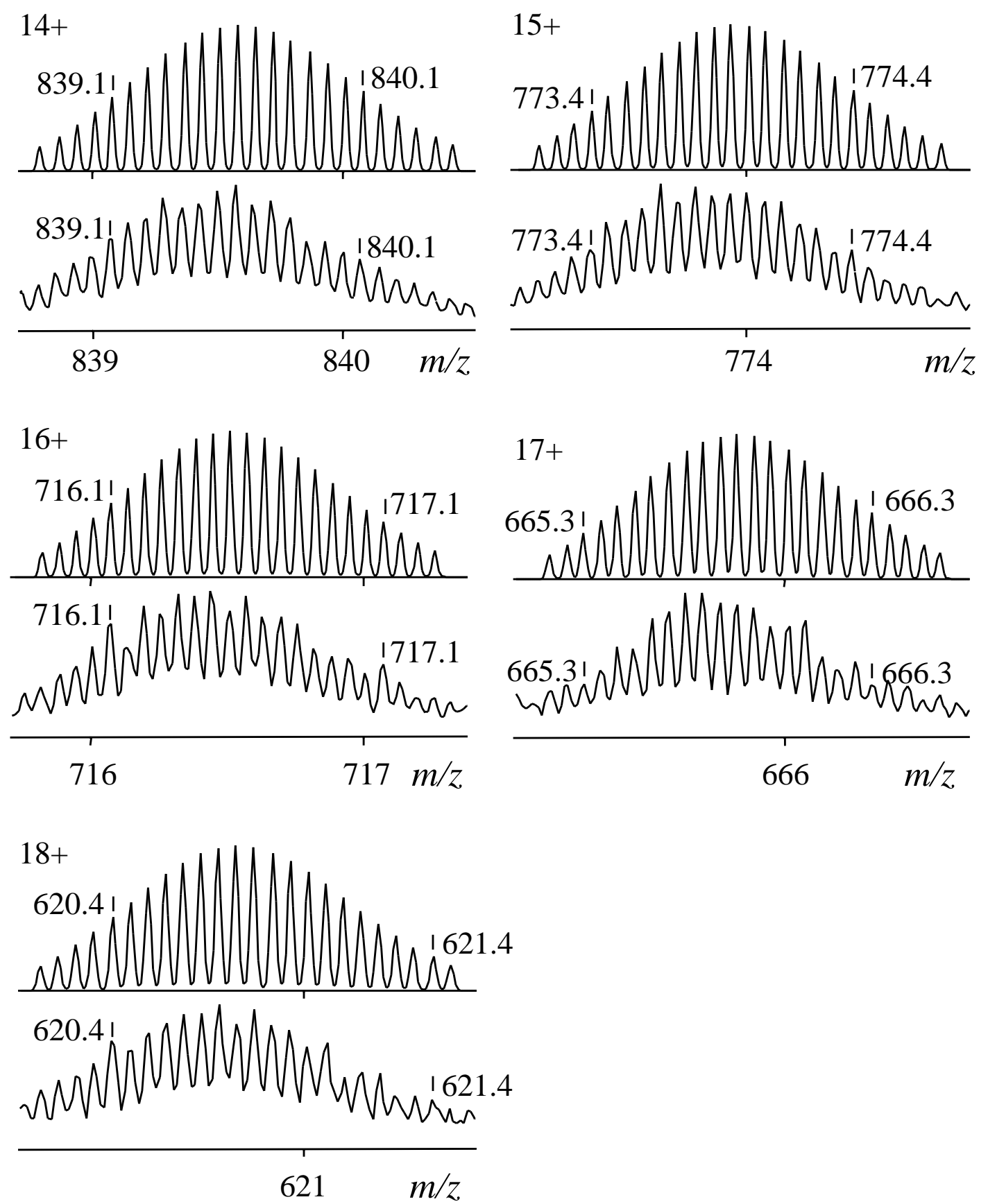

Figure S1. Measured (bottom) and calculated (top) isotope patterns for different charge states observed from $\left[\mathbf{Z n}_{\mathbf{1 2}} \mathbf{L A}_{\mathbf{6}}\right]\left(\mathrm{PF}_{6}^{-}\right.$as counterion). 

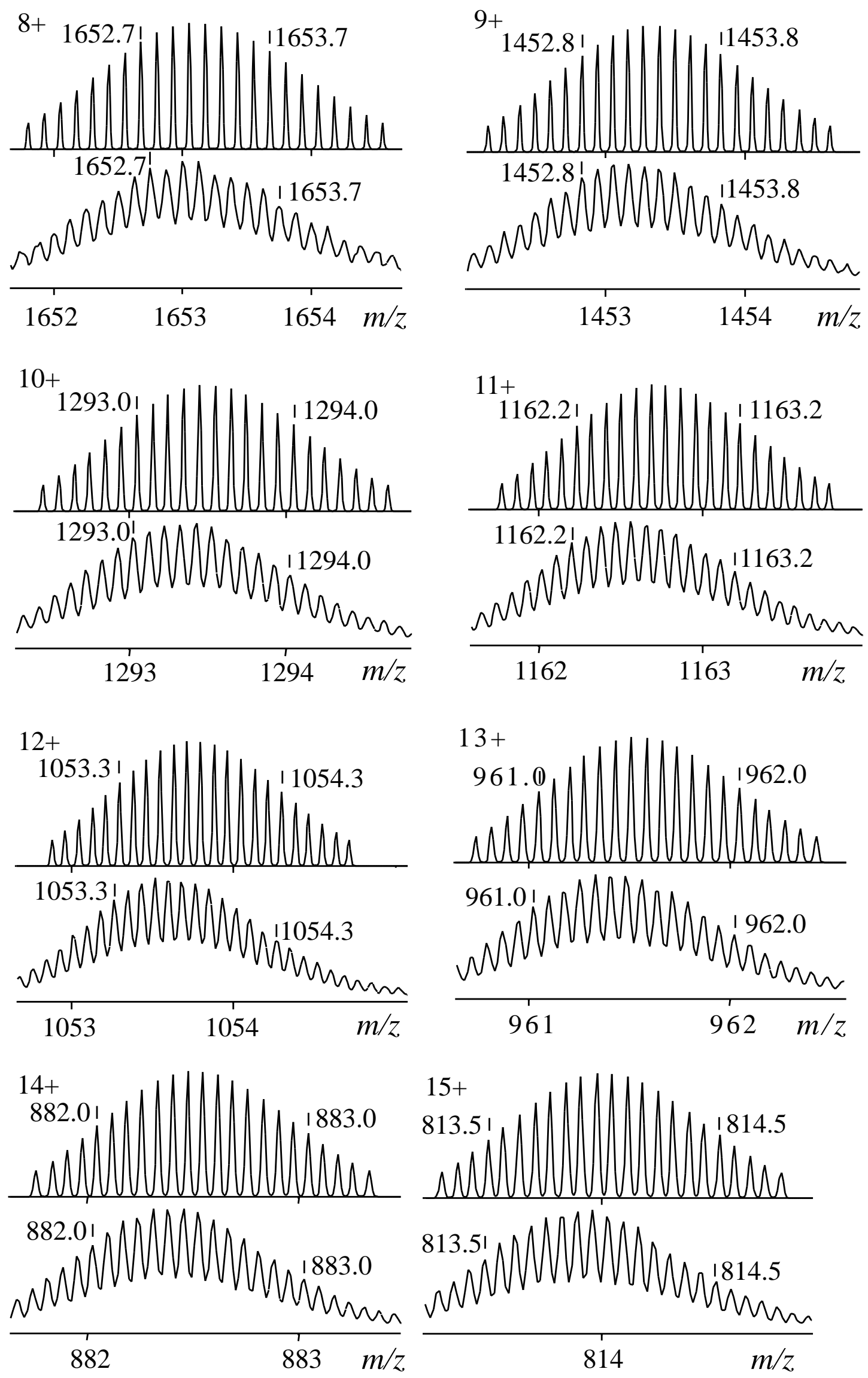

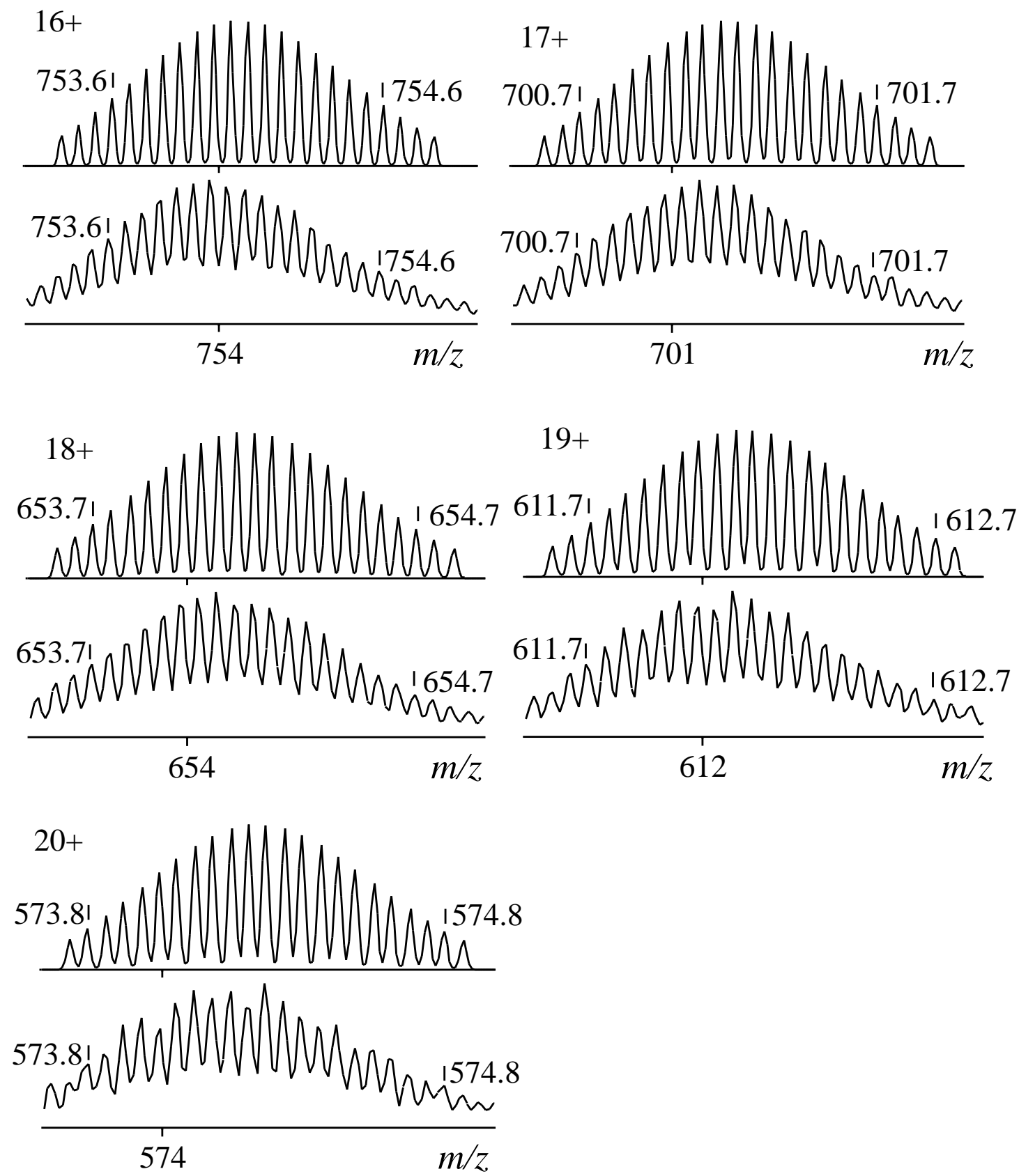

Figure S2. Measured (bottom) and calculated (top) isotope patterns for different charge states observed from $\left[\mathbf{Z n}_{\mathbf{1 2}} \mathbf{L} \mathbf{B}_{\mathbf{6}}\right]$ ( $\mathrm{PF}_{6}{ }^{-}$as counterion). 

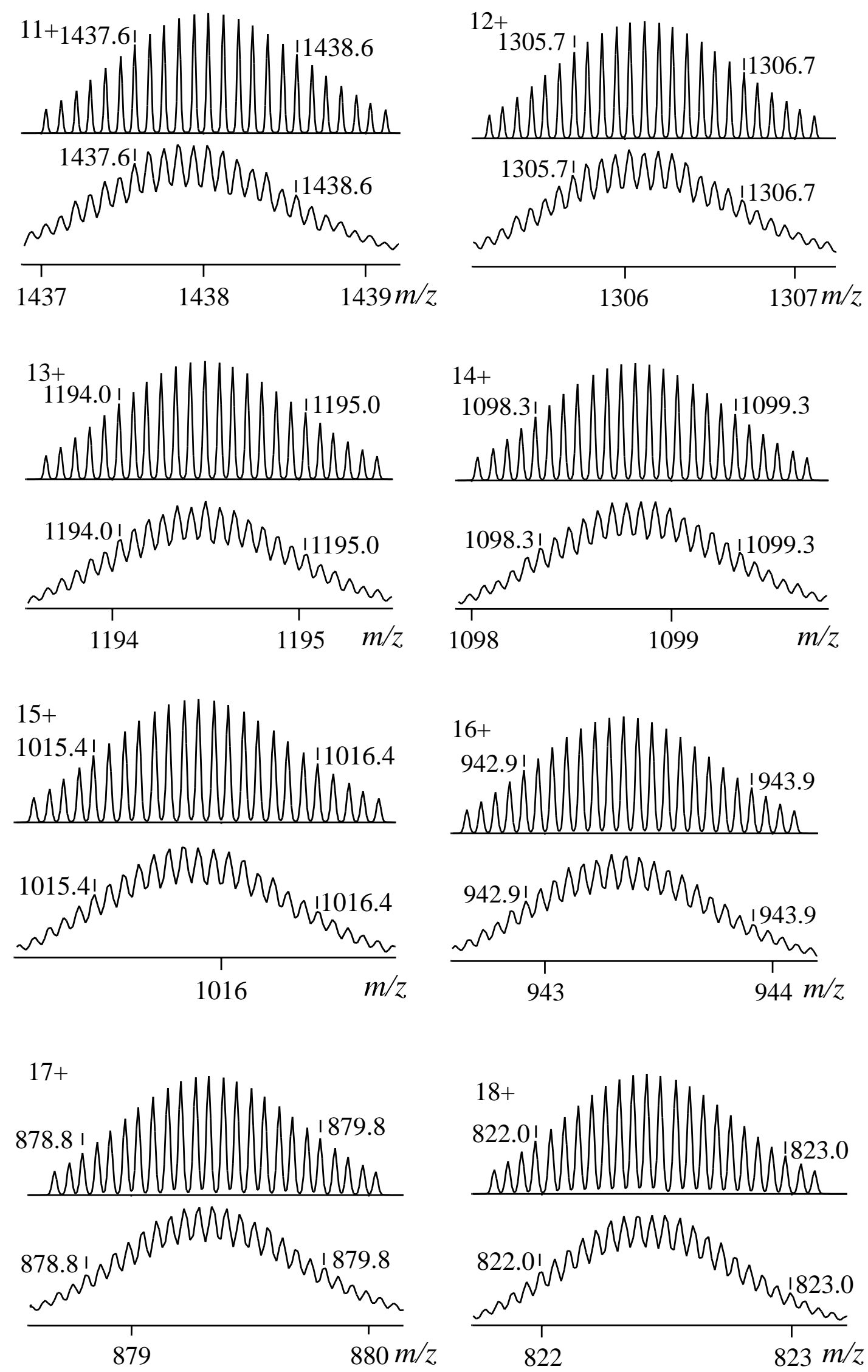

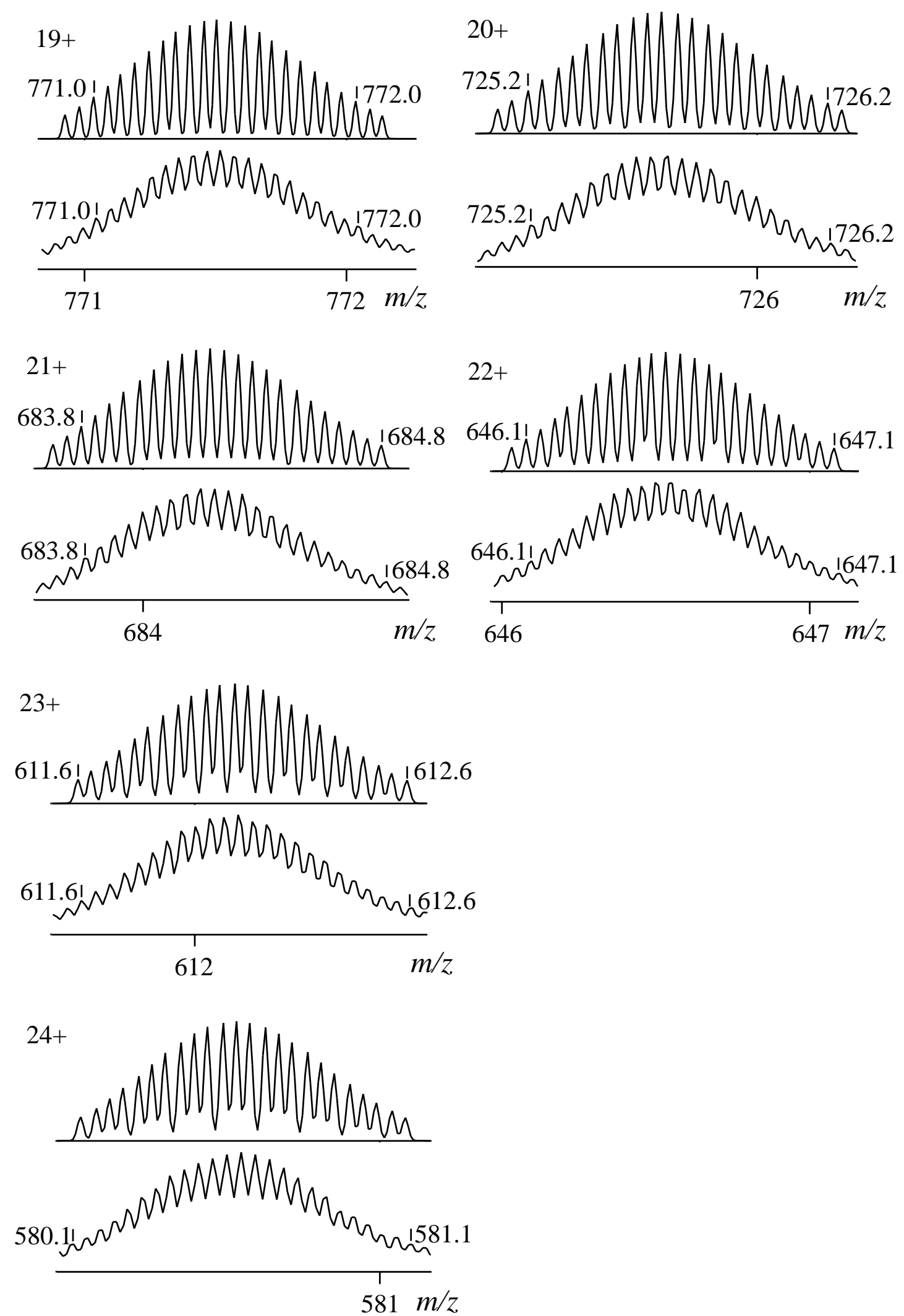

Figure S3. Measured (bottom) and calculated (top) isotope patterns for different charge states observed from $\left[\mathbf{Z n}_{\mathbf{1 2}} \mathbf{L} \mathbf{C}_{\mathbf{6}}\right]\left(\mathrm{PF}_{6}{ }^{-}\right.$as counterion). 

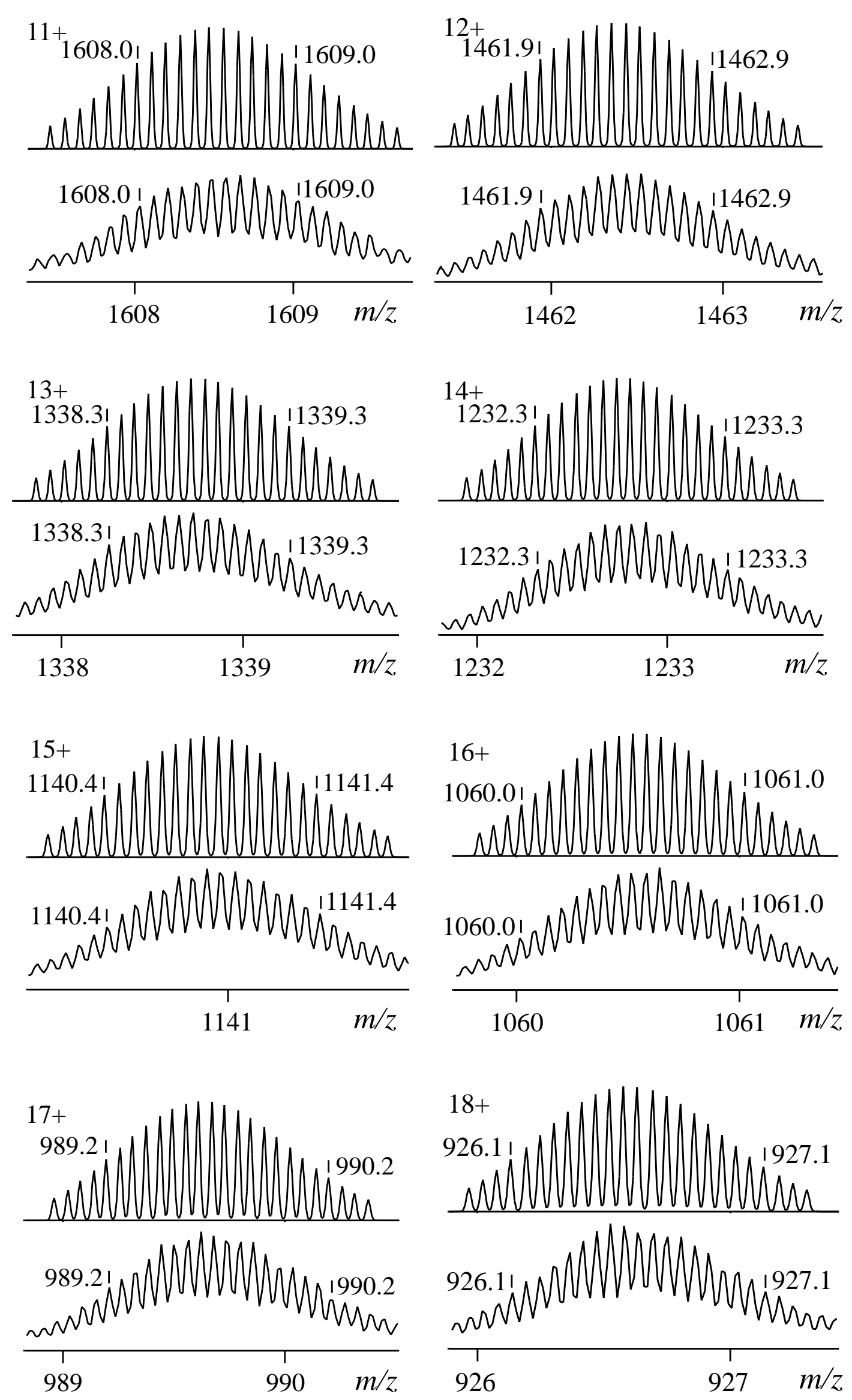

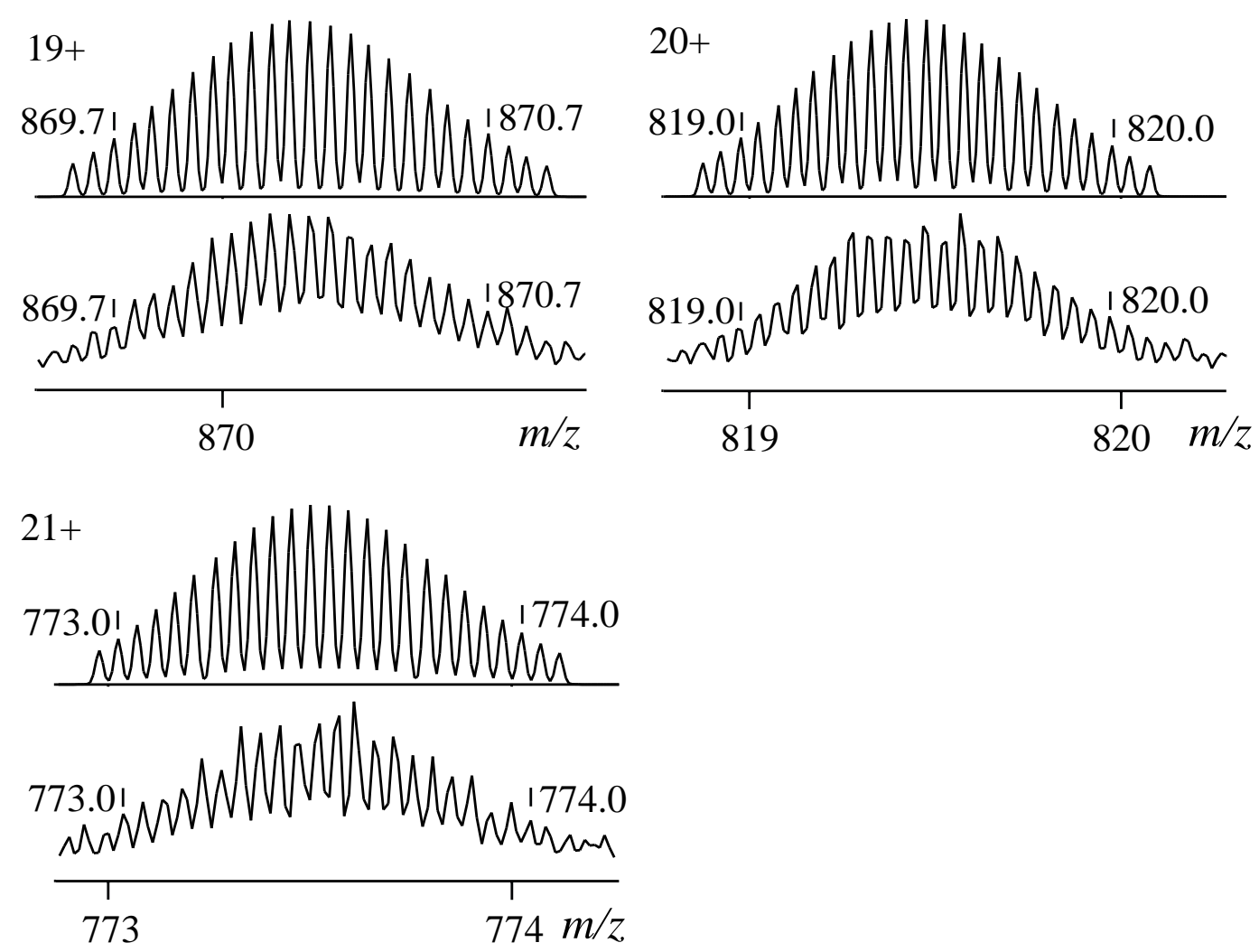

Figure S4. Measured (bottom) and calculated (top) isotope patterns for different charge states observed from $\left[\mathbf{Z n}_{\mathbf{1 2}} \mathbf{L} \mathbf{D}_{\mathbf{6}}\right]\left(\mathrm{PF}_{6}^{-}\right.$as counterion). 
5. Energy-minimized structure from molecular modeling, ESI-MS and TWIM-MS plots of complex [ $\left.\mathrm{Zn}_{12} \mathrm{LA}_{6}\right]$.
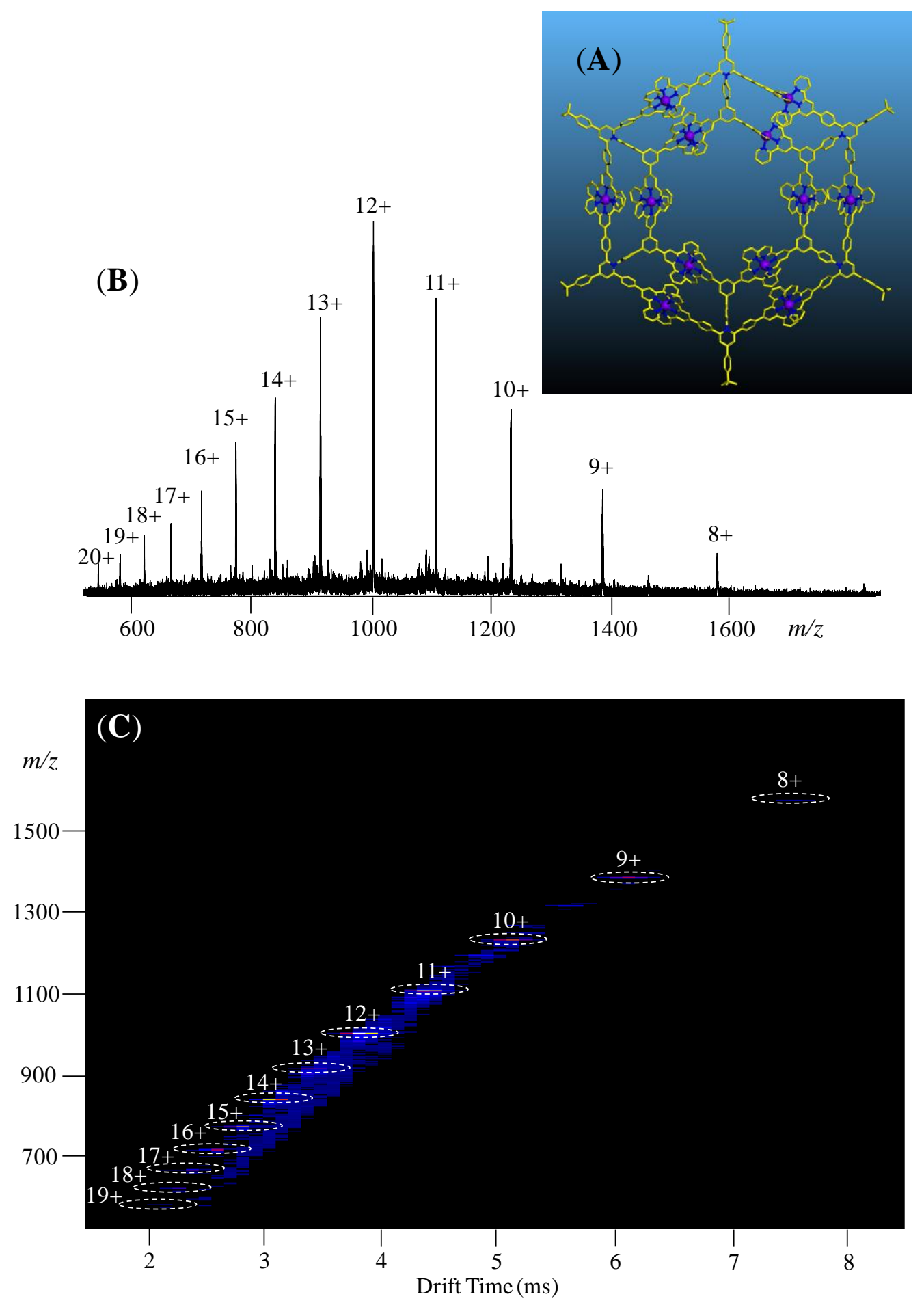

Figure S5. Energy-minimized structure from molecular modeling of complex [Zn $\left.\mathbf{n}_{12} \mathbf{L A}_{\mathbf{6}}\right]$. (B) ESI-MS and (C) 2D TWIM-MS plot ( $\mathrm{m} / \mathrm{z}$ vs drift time). The charge states of intact assemblies are marked. 


\section{Calibration of drift time scale}

Corrected drift times (arrival times) were plotted against corrected published cross sections for the multiply charged ions arising from myoglobin. Drift times were measured at a traveling wave velocity of $1000 \mathrm{~m} / \mathrm{s}$ and a traveling wave height of 25 V. This calibration plot was utilized to obtain the experimental collision cross sections (CCSs) listed in Table 1.

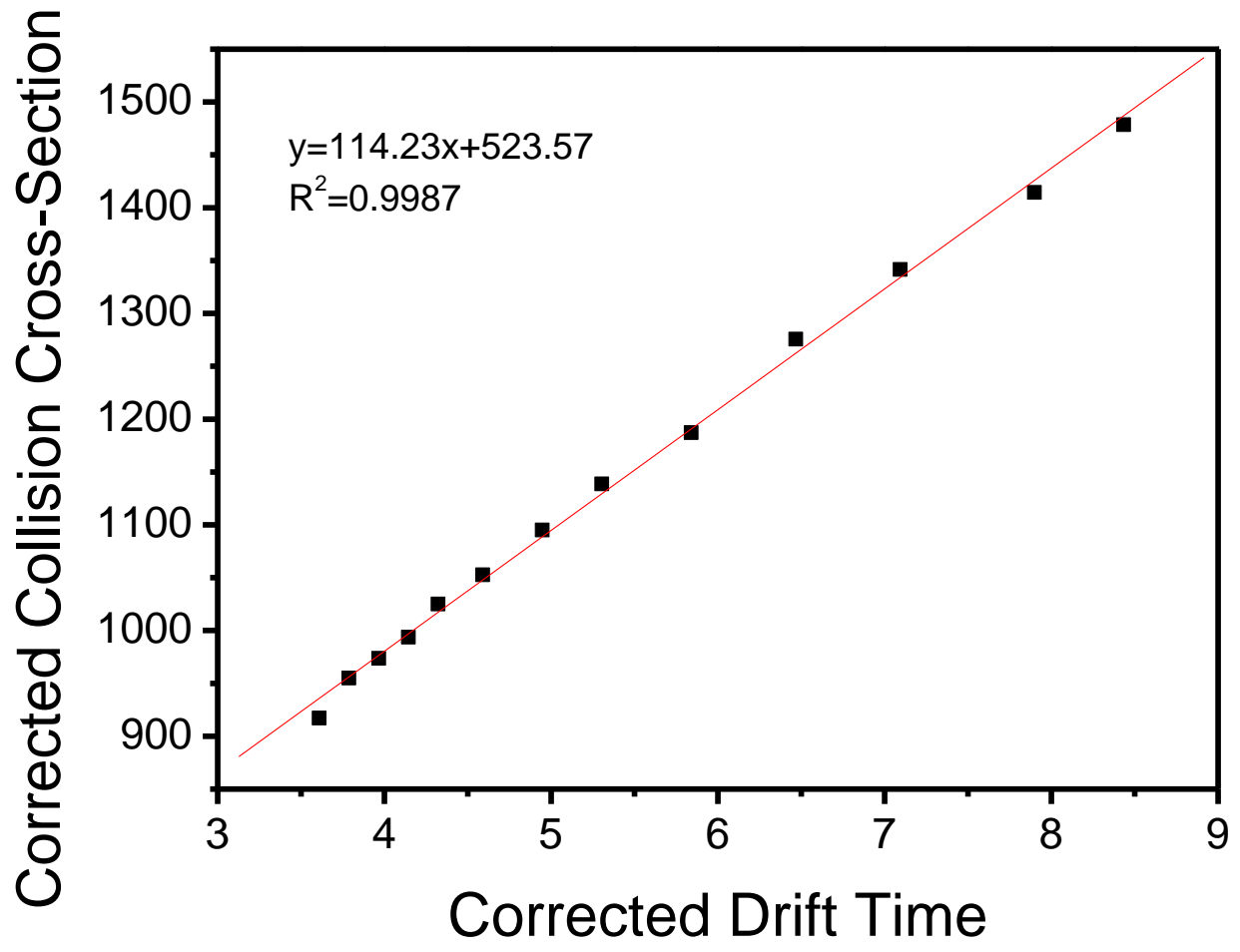

Figure S6. The calibration curve was constructed by plotting the corrected CCSs of the molecular ions of myoglobin at different corrected drift times. 
7. Molecular modeling

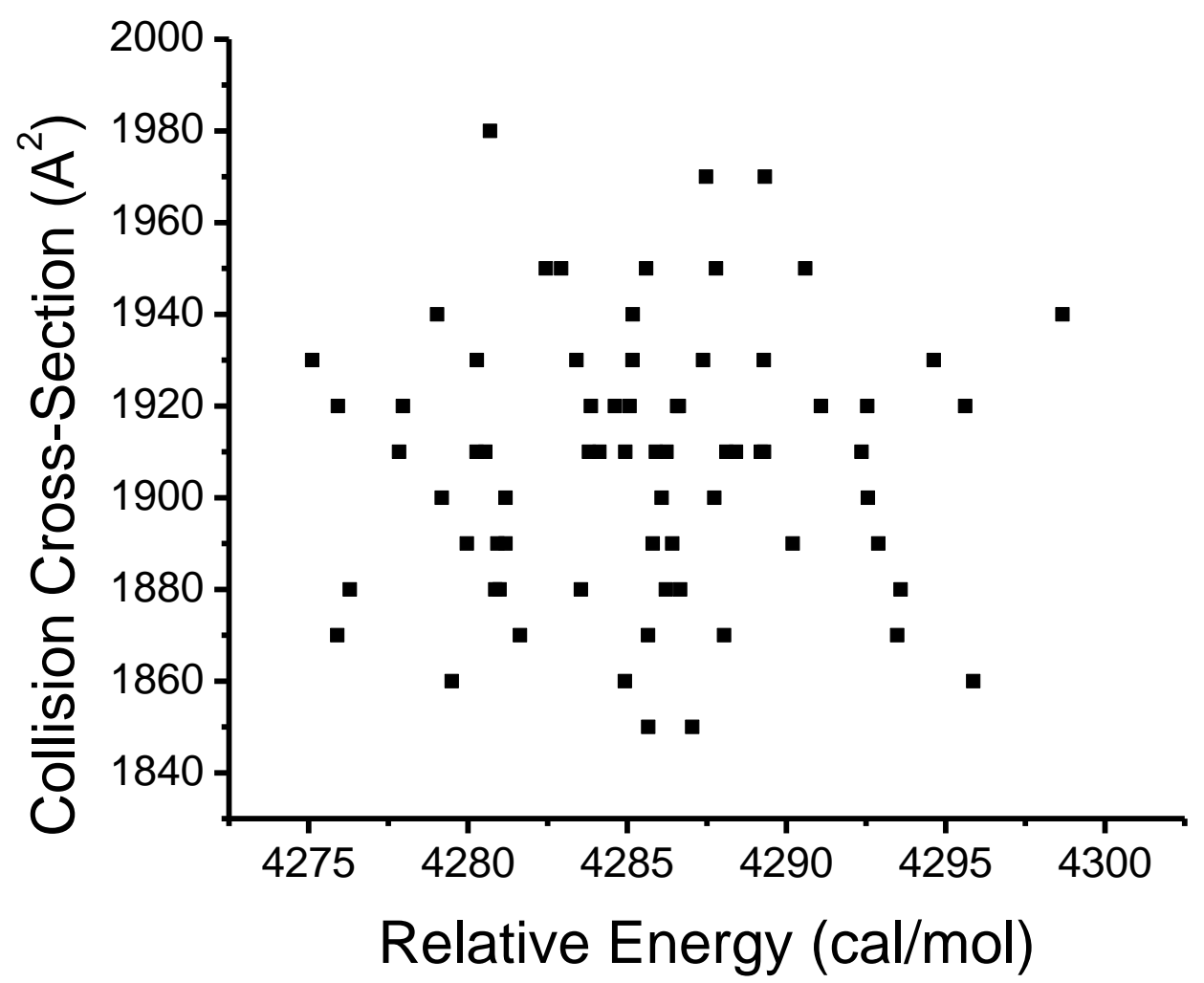

Figure S7. Plot of collision cross-section (CCS) vs. relative energy for 70 candidate structures of $\left[\mathbf{Z n}_{12} \mathbf{L B}_{\mathbf{6}}\right]$ generated by annealing simulations. CCSs were calculated by the TM method using the MOBCAL program. The average TM cross section area is $1907.7 \pm 29.8 \AA^{2}$. 


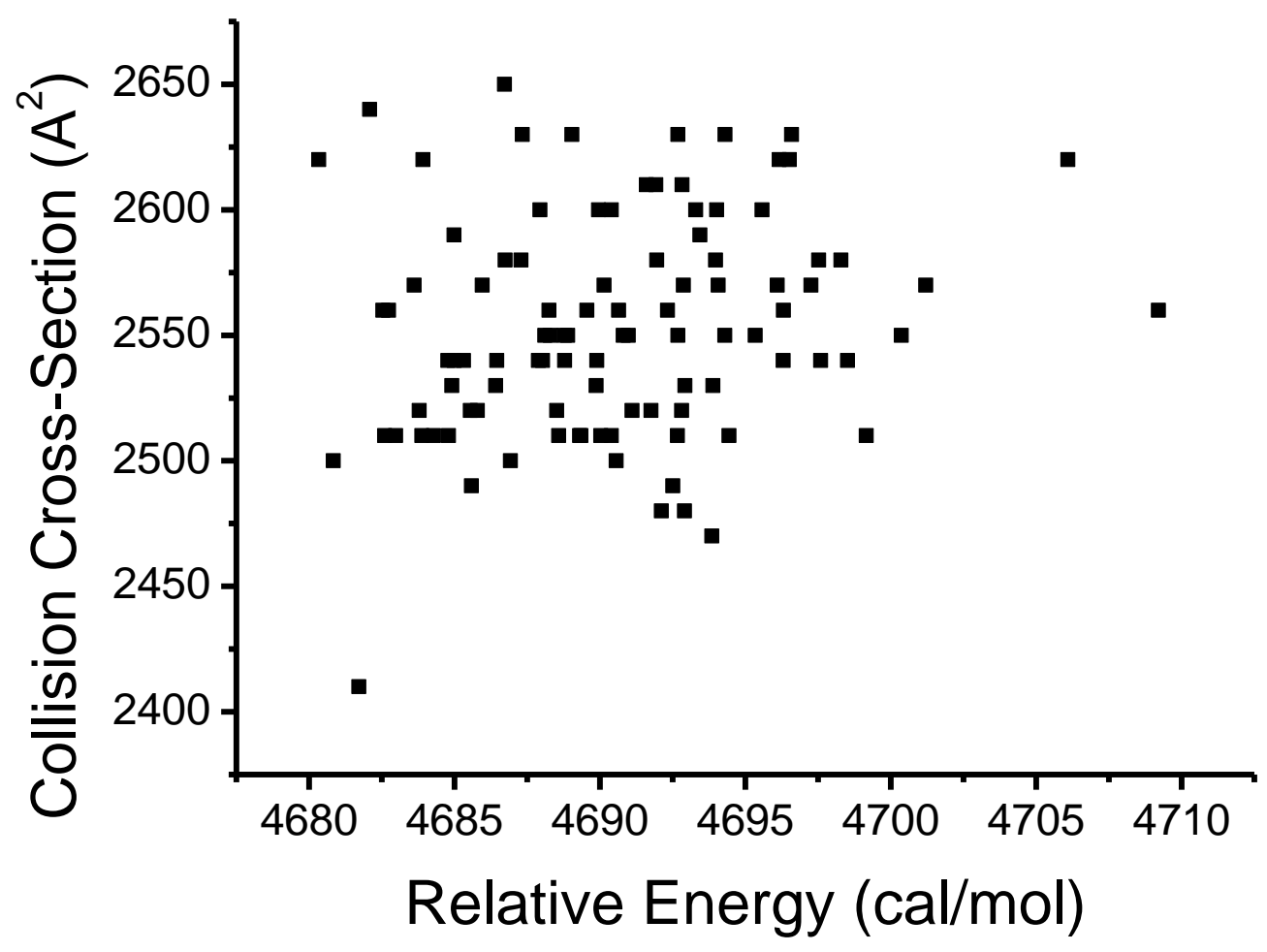

Figure S8. Plot of collision cross-section (CCS) vs. relative energy for 100 candidate structures of $\left[\mathbf{Z n}_{12} \mathbf{L} \mathbf{C}_{6}\right]$ generated by annealing simulations. CCSs were calculated by the TM method using the MOBCAL program. The average TM cross section area is $2554.2 \pm 44.1 \AA^{2}$. 


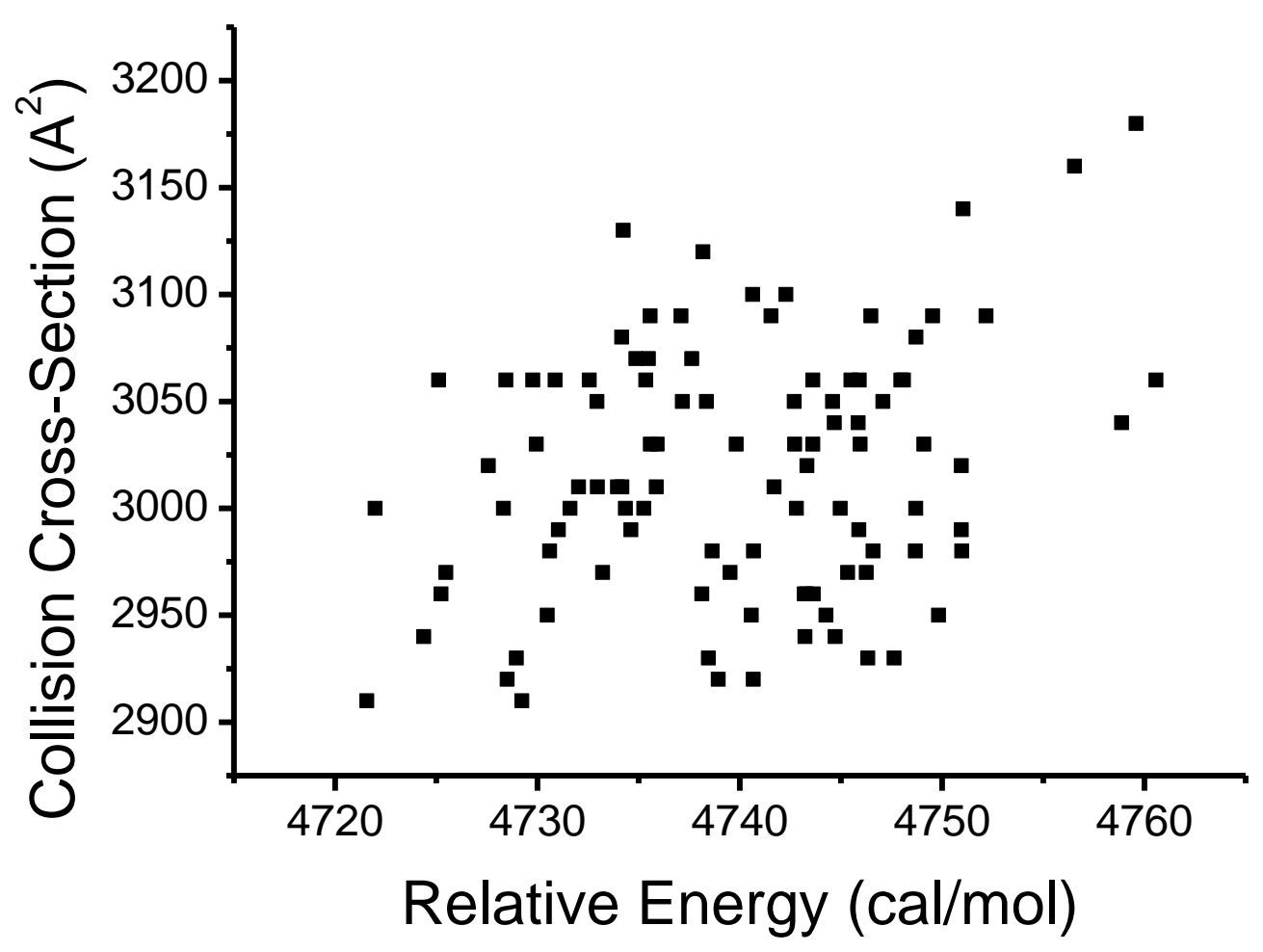

Figure S9. Plot of collision cross-section (CCS) vs. relative energy for 100 candidate structures of [ $\left.\mathbf{Z n}_{12} \mathbf{L} \mathbf{D}_{\mathbf{6}}\right]$ generated by annealing simulations. CCSs were calculated by the TM method using the MOBCAL program. The average TM cross section area is $3018.9 \pm 58.3 \AA^{2}$. 
8. ${ }^{1} \mathrm{H}$ NMR, ${ }^{13} \mathrm{C}$ NMR, 2D COSY NMR, 2D NOESY NMR and MALDI-TOF.

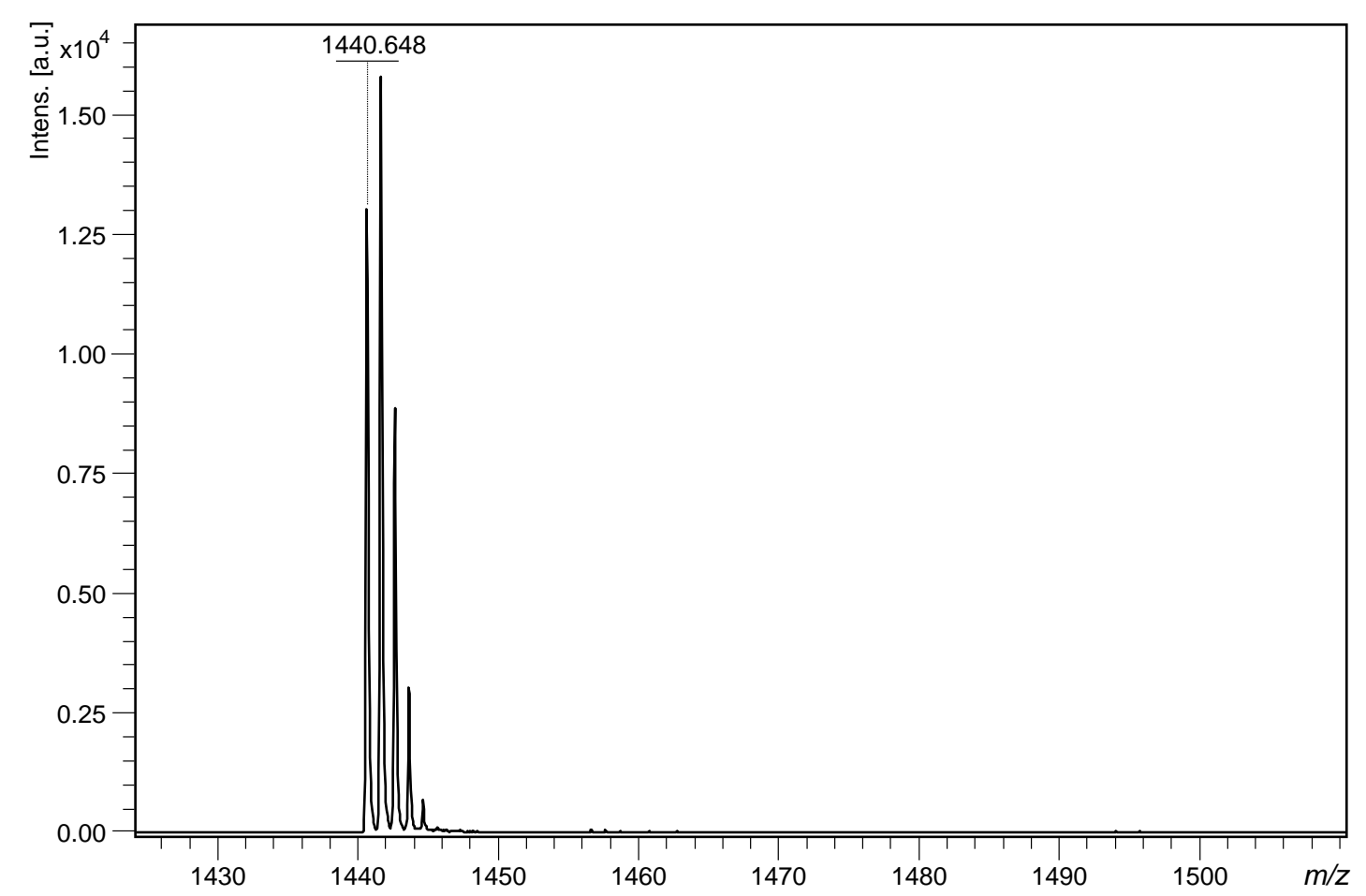

Figure S10. MALDI-TOF mass spectrum of ligand LA.

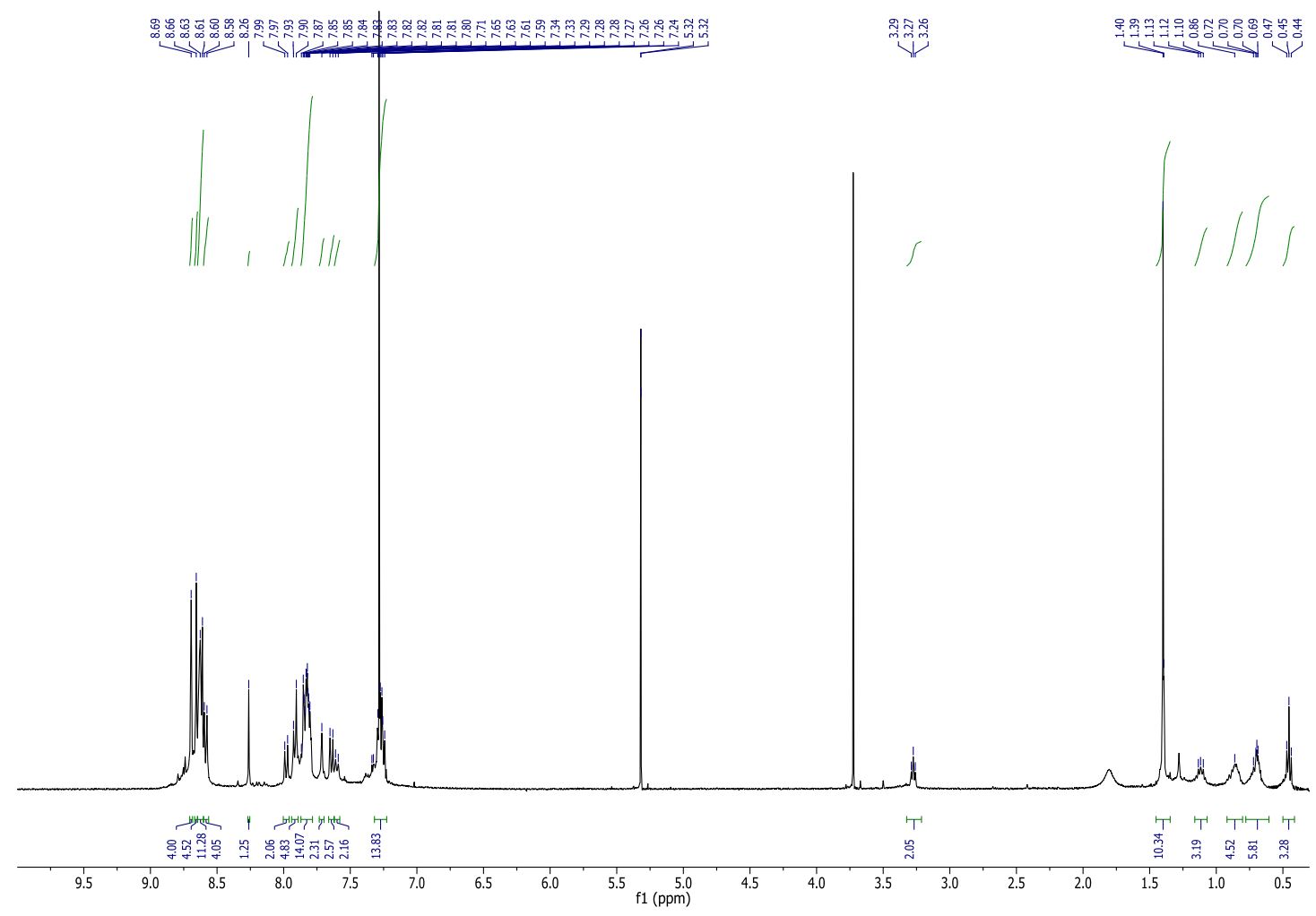

Figure S11. ${ }^{1} \mathrm{H}$ NMR (400 MHz) spectrum of ligand LB. 


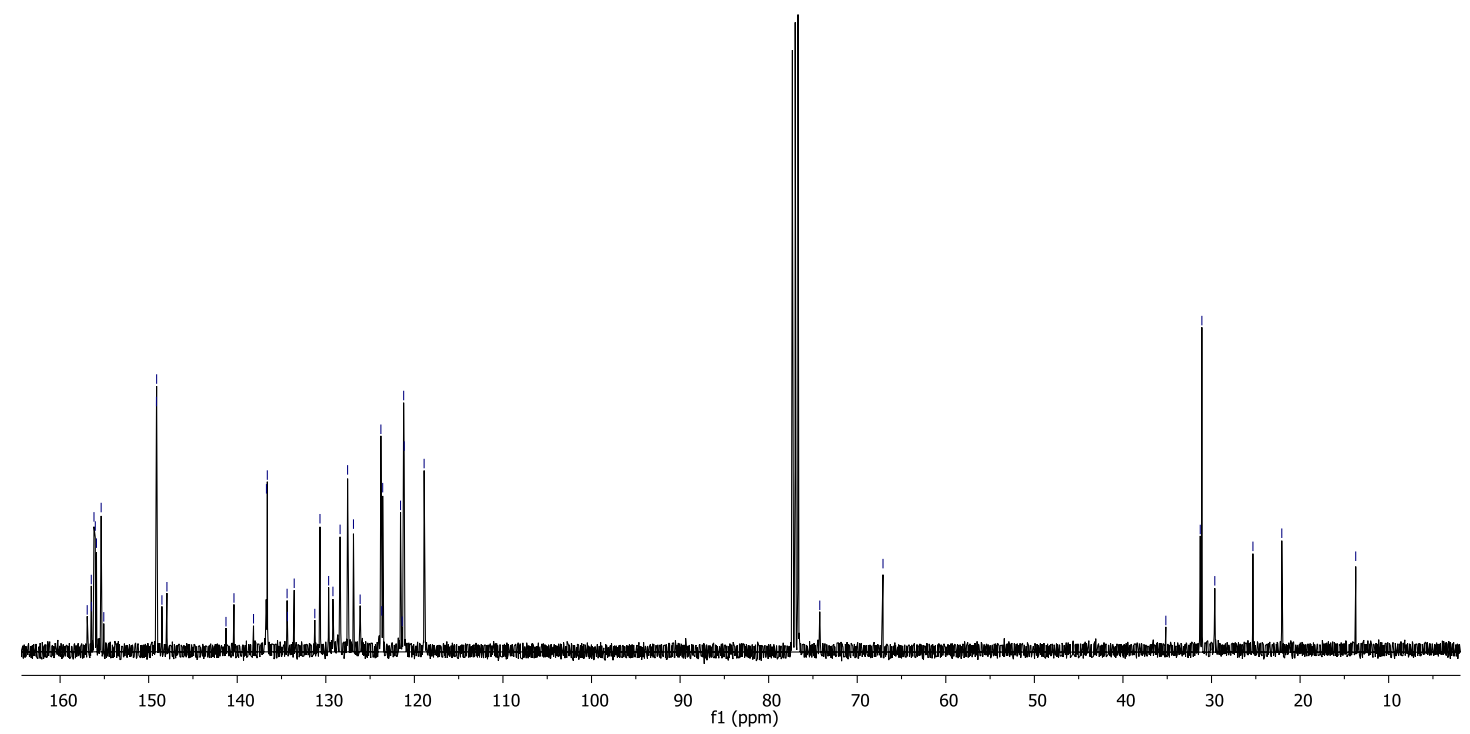

Figure S12. ${ }^{13} \mathrm{C}$ NMR (400 MHz) spectrum of ligand LB.

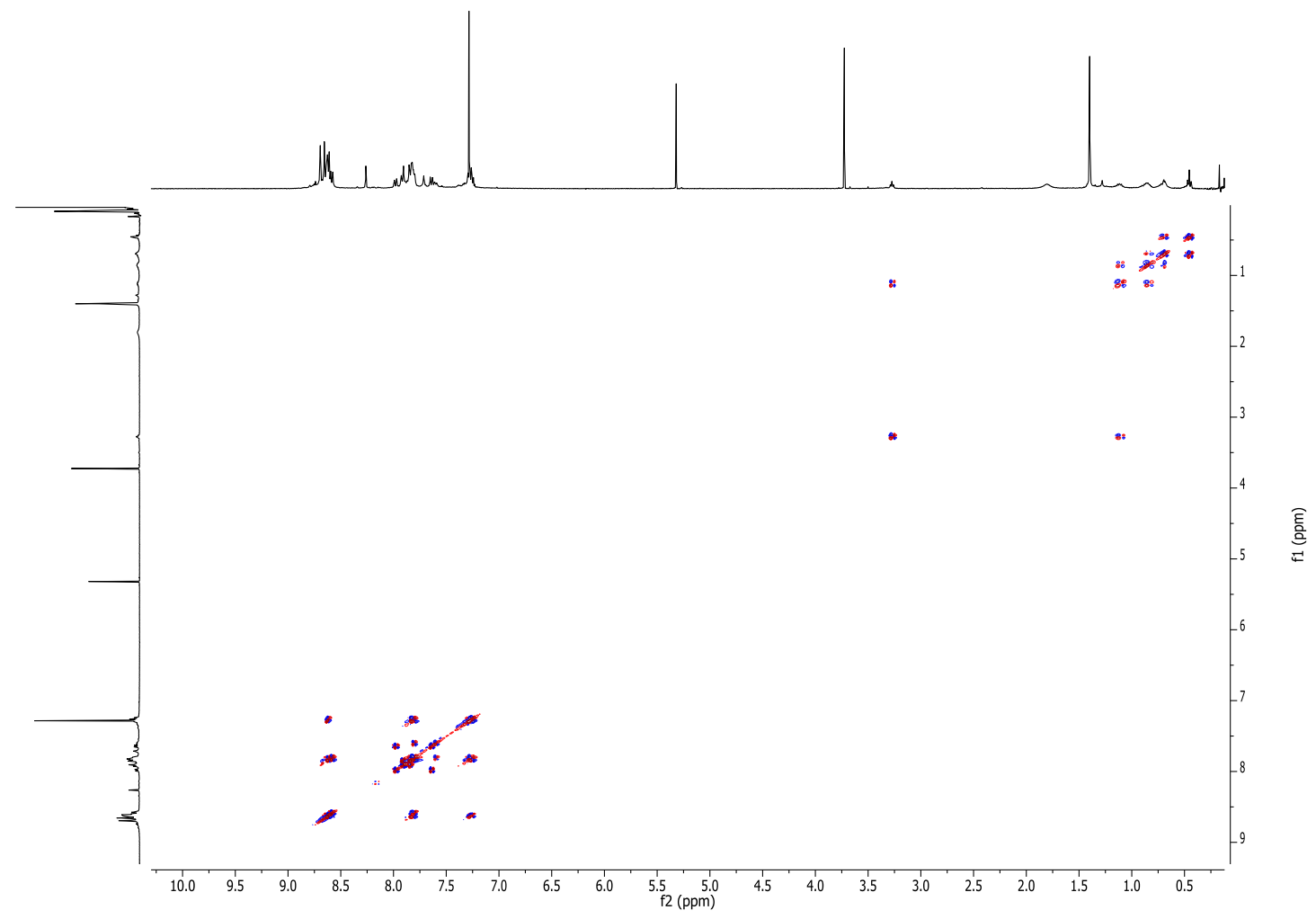

Figure S13. 2D COSY NMR (400 MHz) spectrum of ligand LB. 


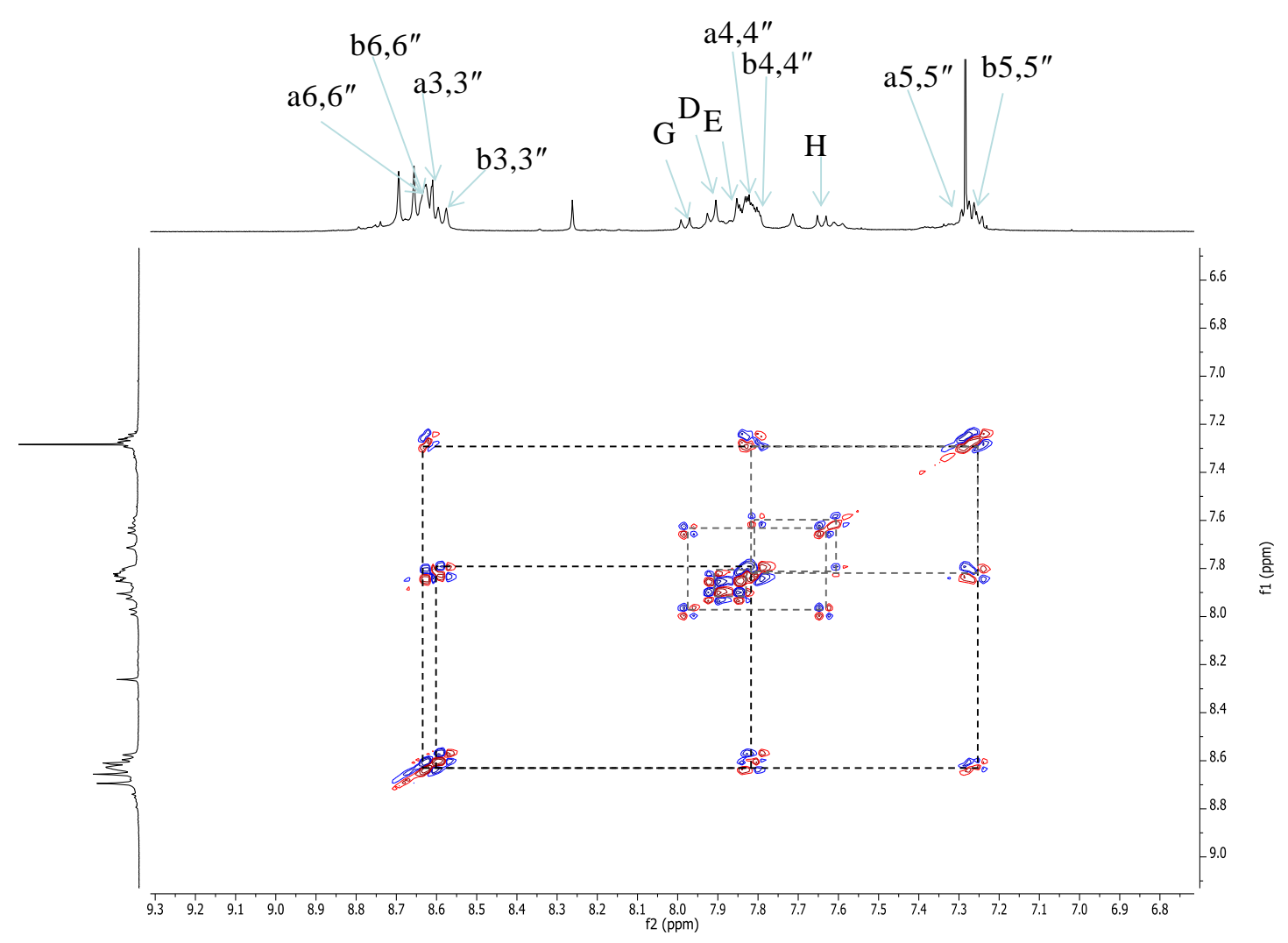

Figure S14. 2D COSY NMR (400 MHz) spectrum of ligand LB (aromatic region).

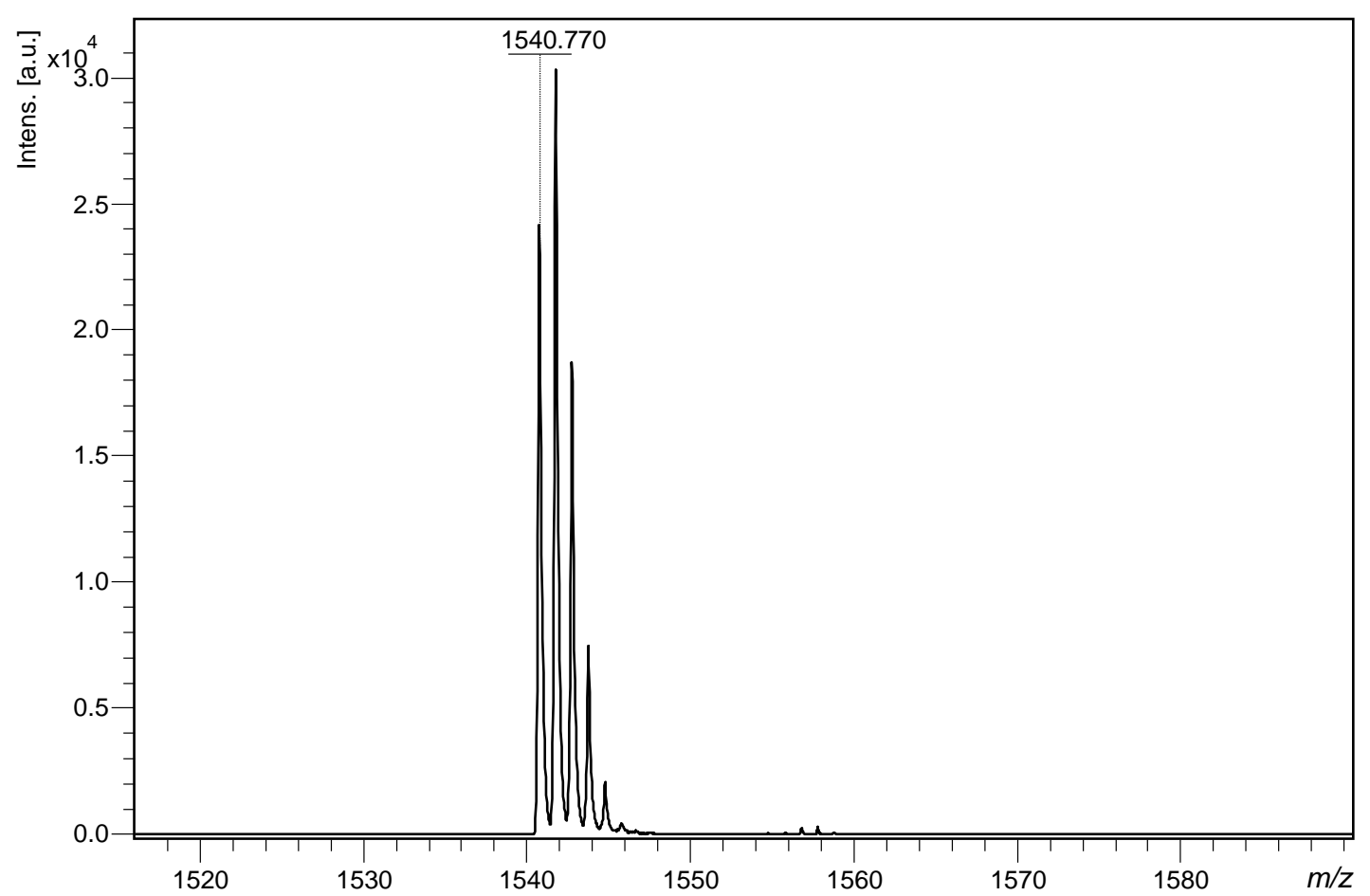

Figure S15. MALDI-TOF mass spectrum of ligand LB. 

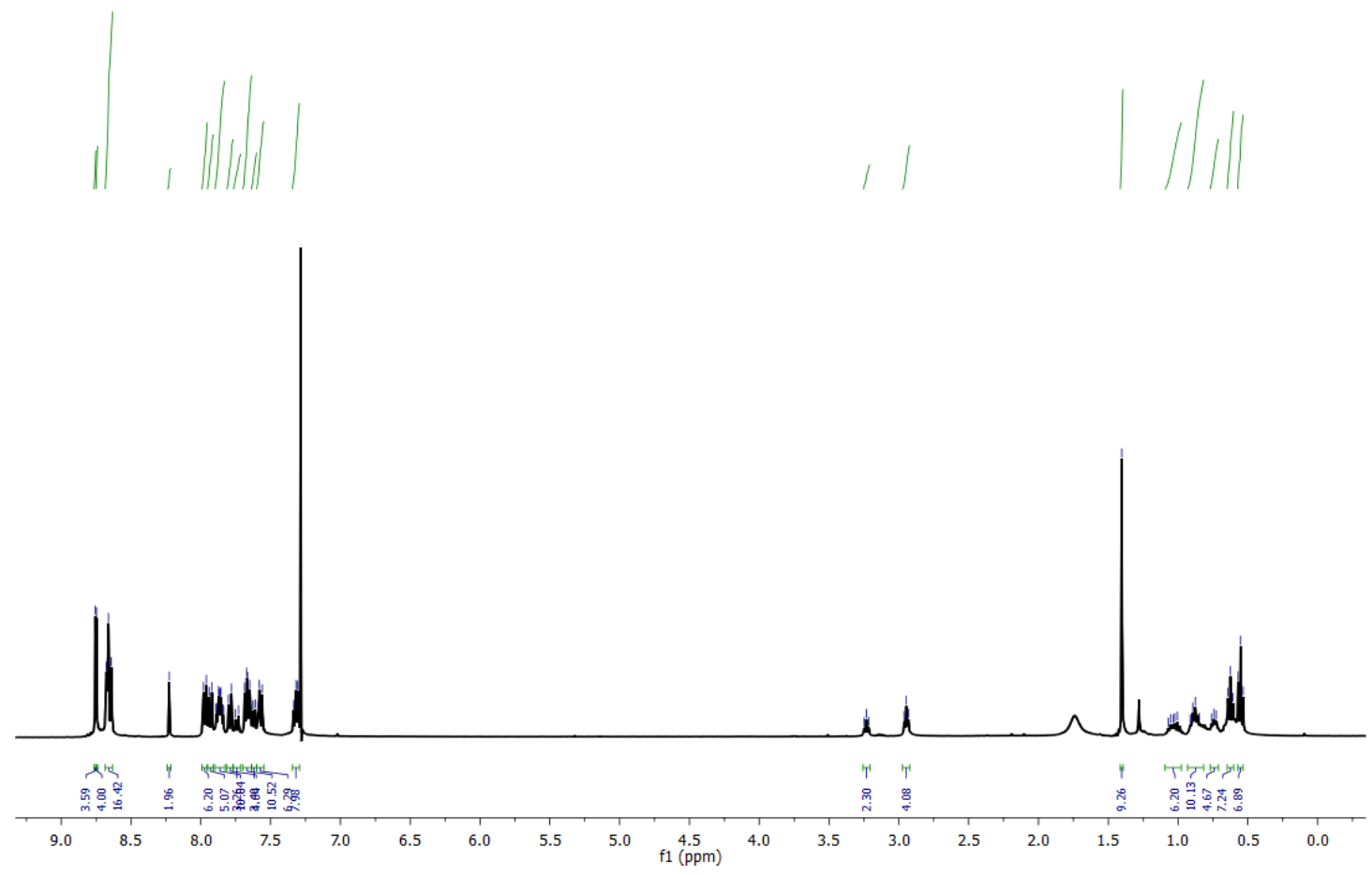

Figure S16. ${ }^{1} \mathrm{H}$ NMR (400 MHz) spectrum of ligand LC.

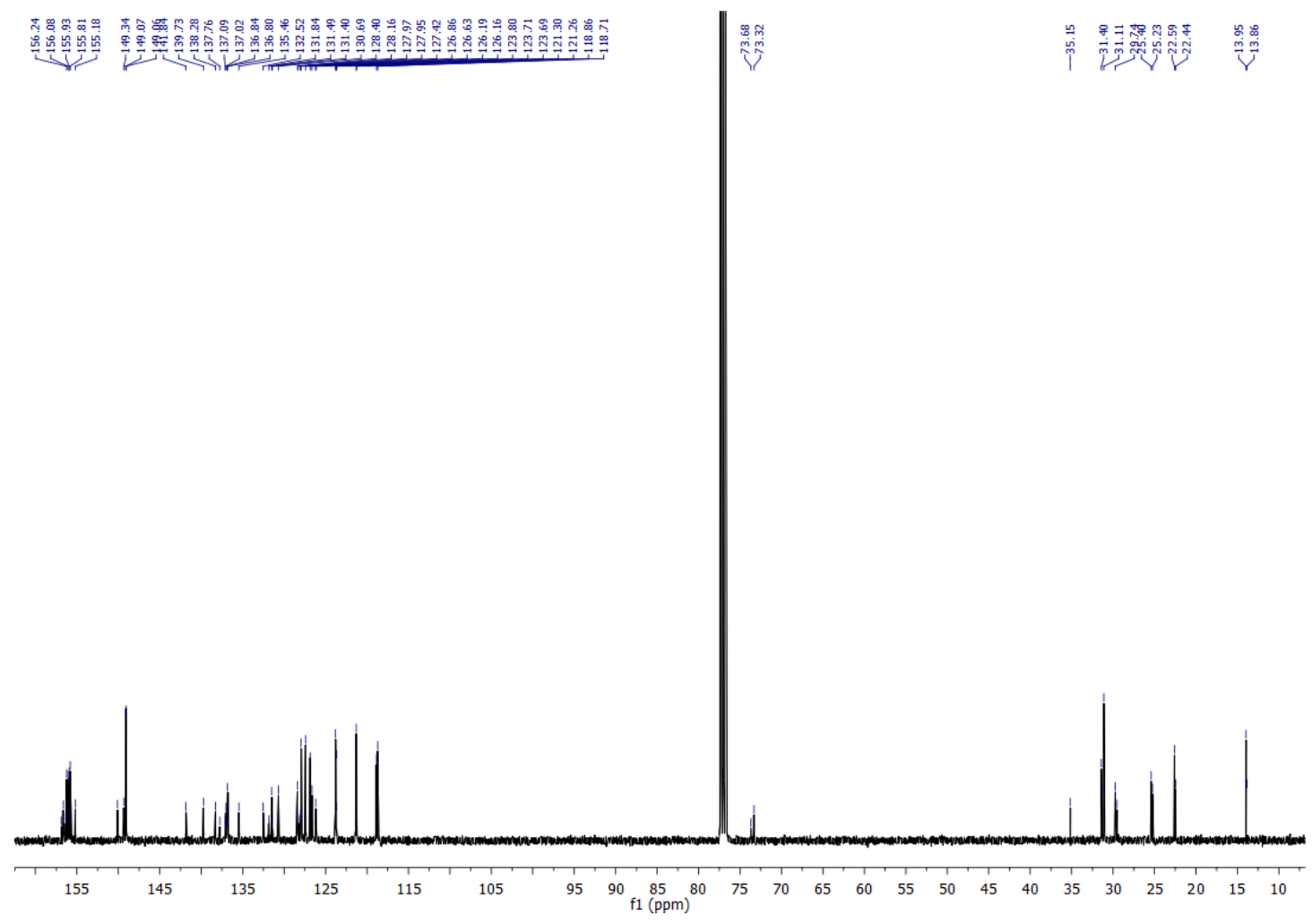

Figure S17. ${ }^{13} \mathrm{C}$ NMR (400 MHz) spectrum of ligand LC. 


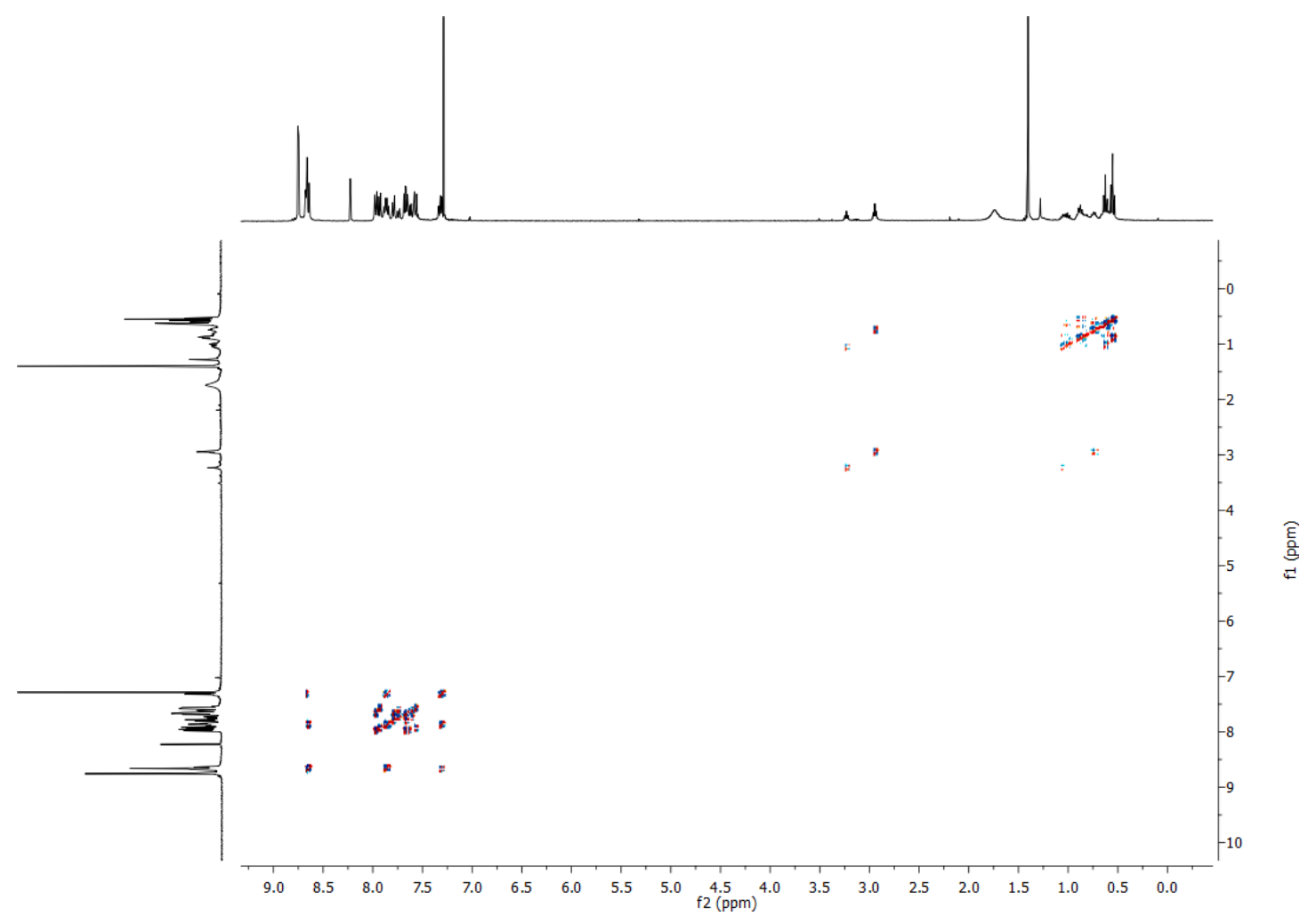

Figure S18.2D COSY NMR (400 MHz) spectrum of ligand LC.

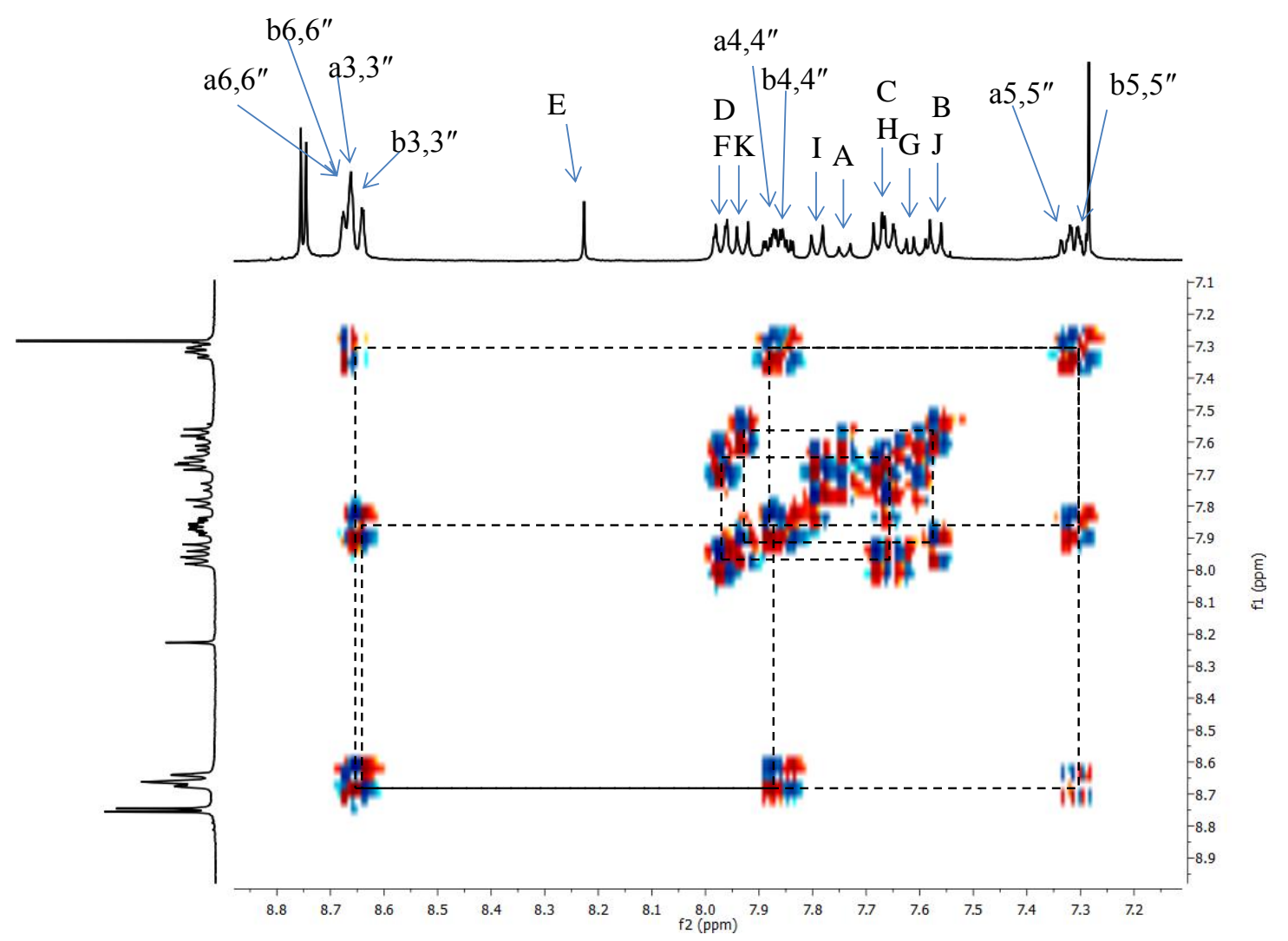

Figure S19. 2D COSY NMR (400 MHz) spectrum of ligand LC (aromatic region). 


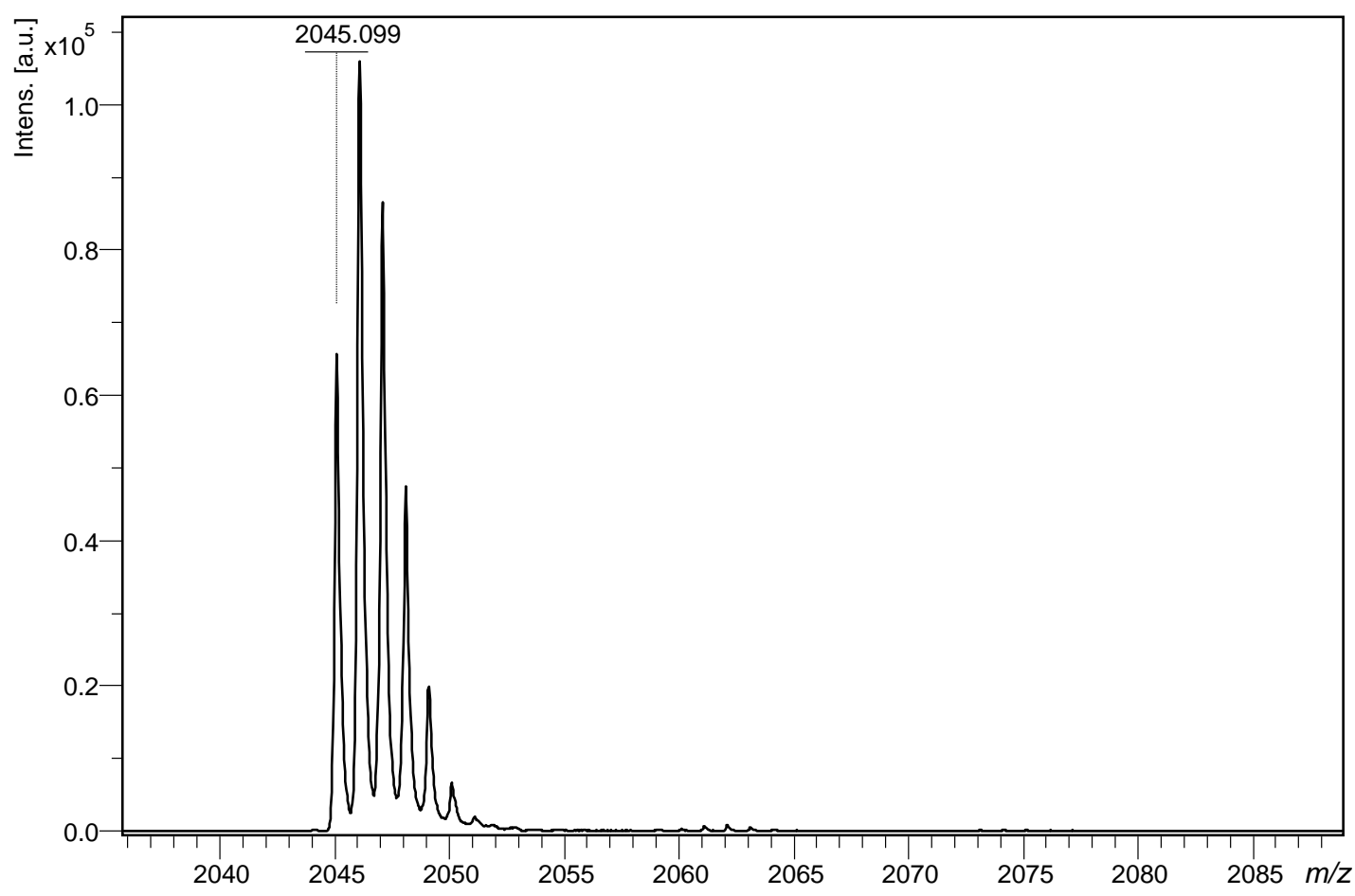

Figure S20. MALDI-TOF mass spectrum of ligand LC.

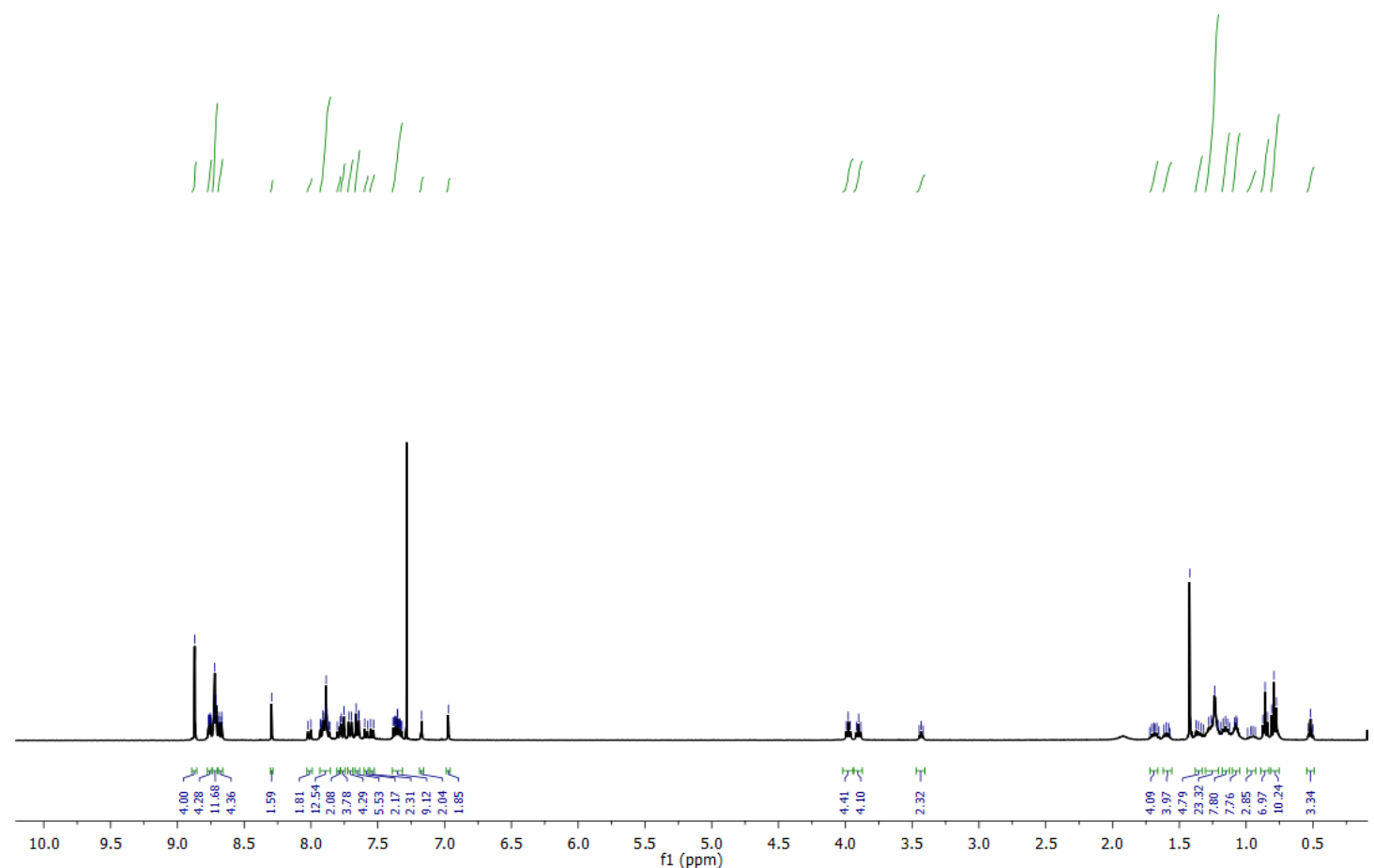

Figure S21. ${ }^{1} \mathrm{H}$ NMR (400 MHz) spectrum of ligand LD. 


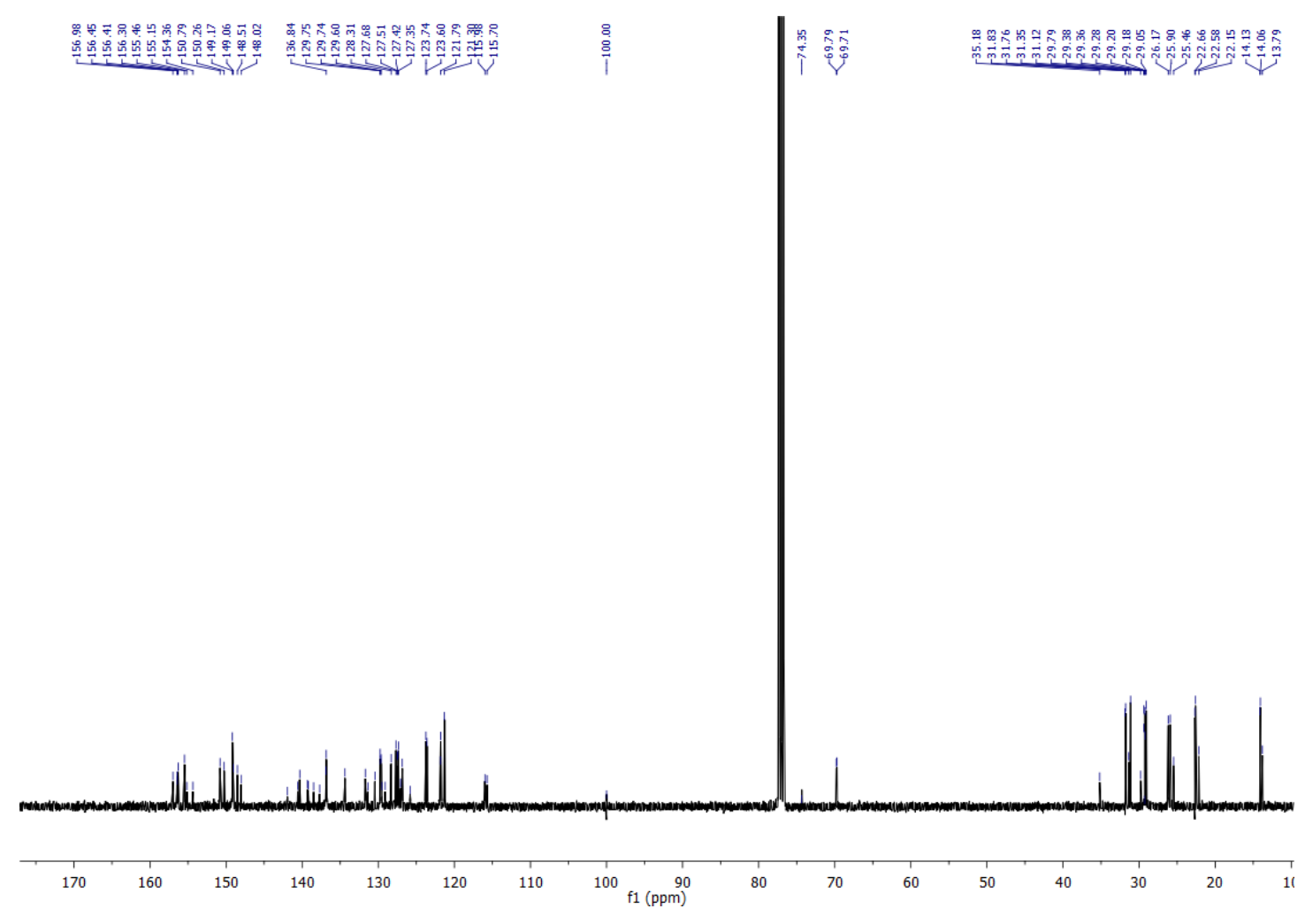

Figure S22. ${ }^{13} \mathrm{C}$ NMR (400 MHz) spectrum of ligand LD.

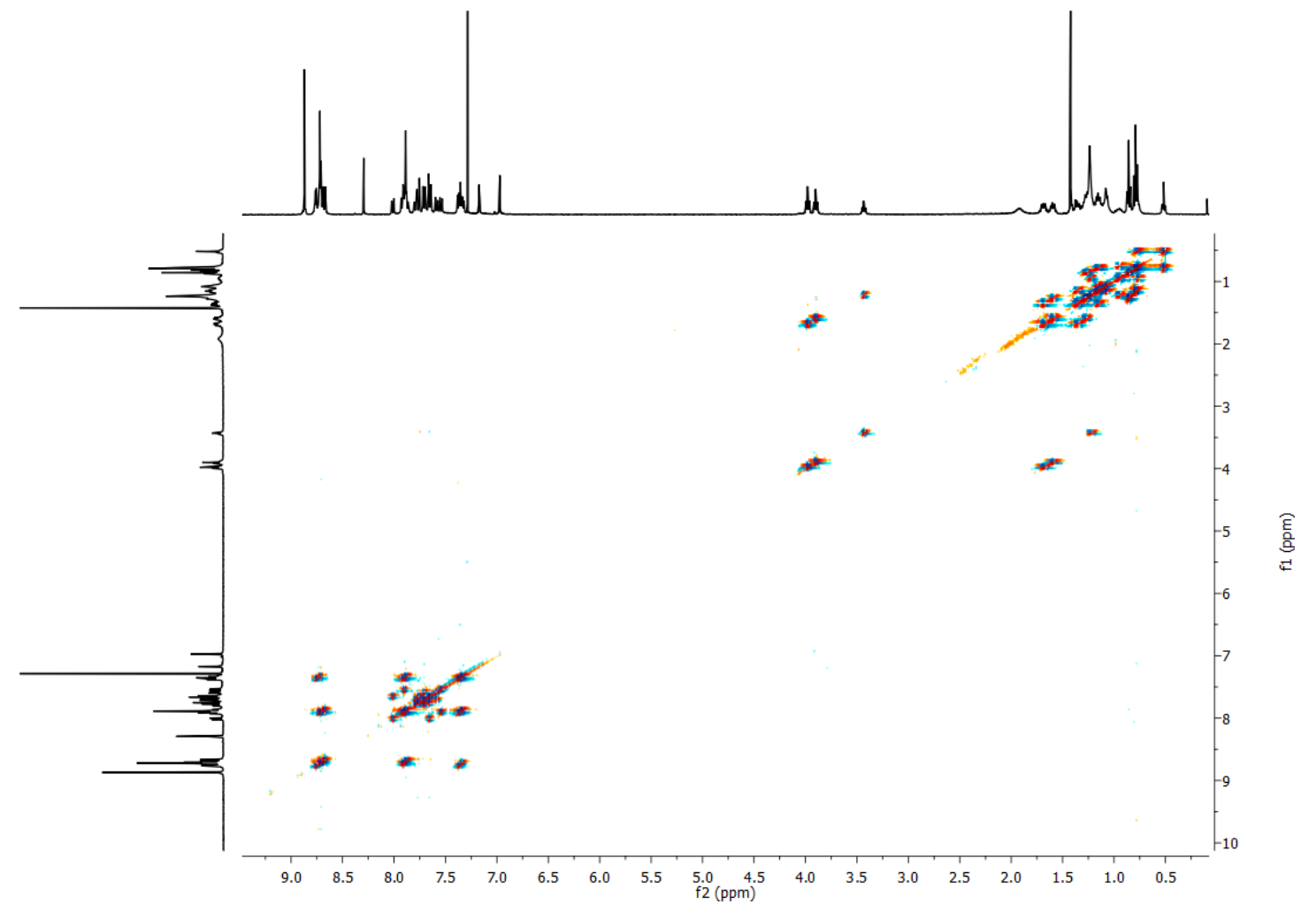

Figure S23.2D COSY NMR (400 MHz) spectrum of ligand LD. 


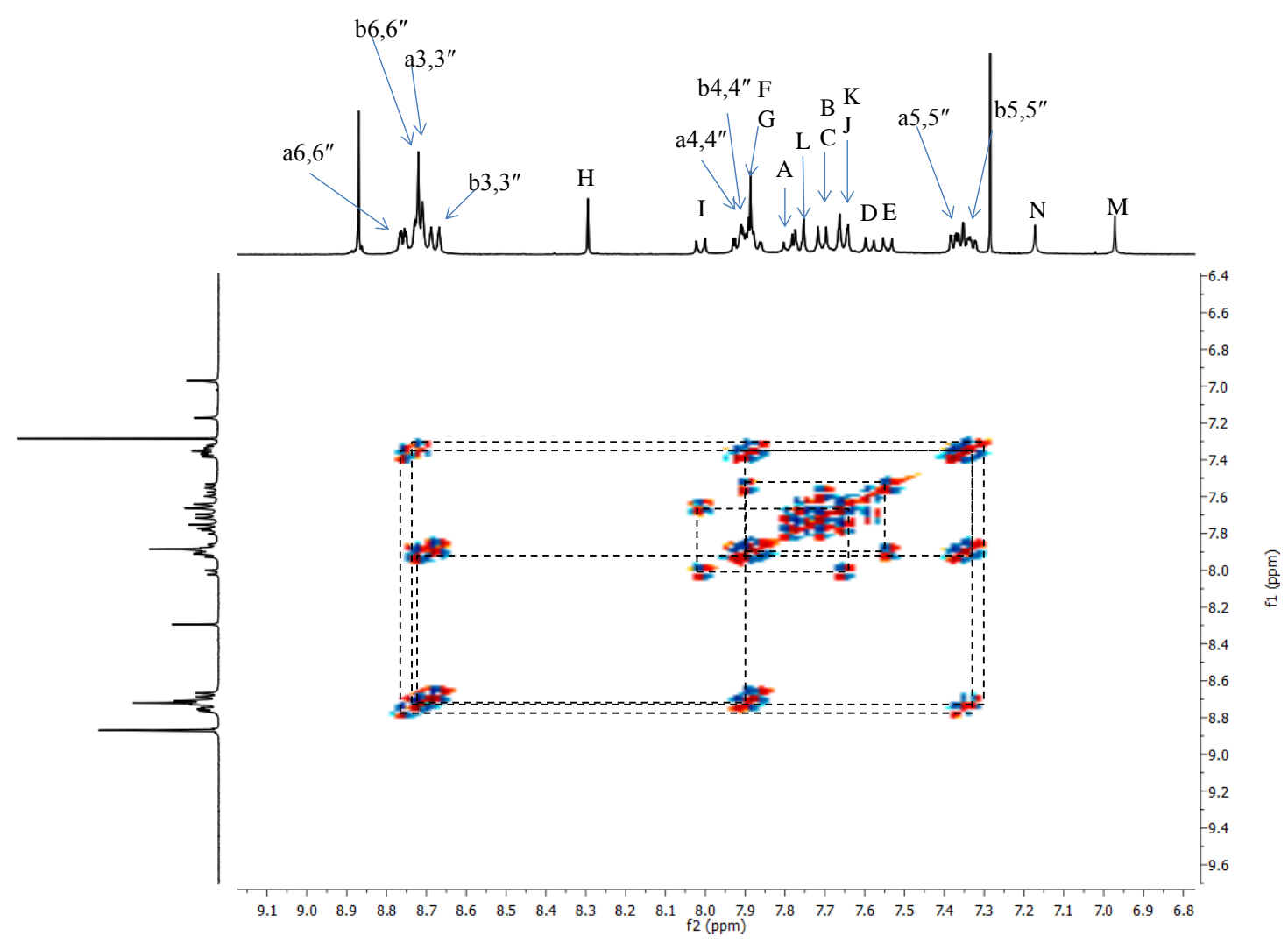

Figure S24. 2D COSY NMR (400 MHz) spectrum of ligand LD (aromatic region).

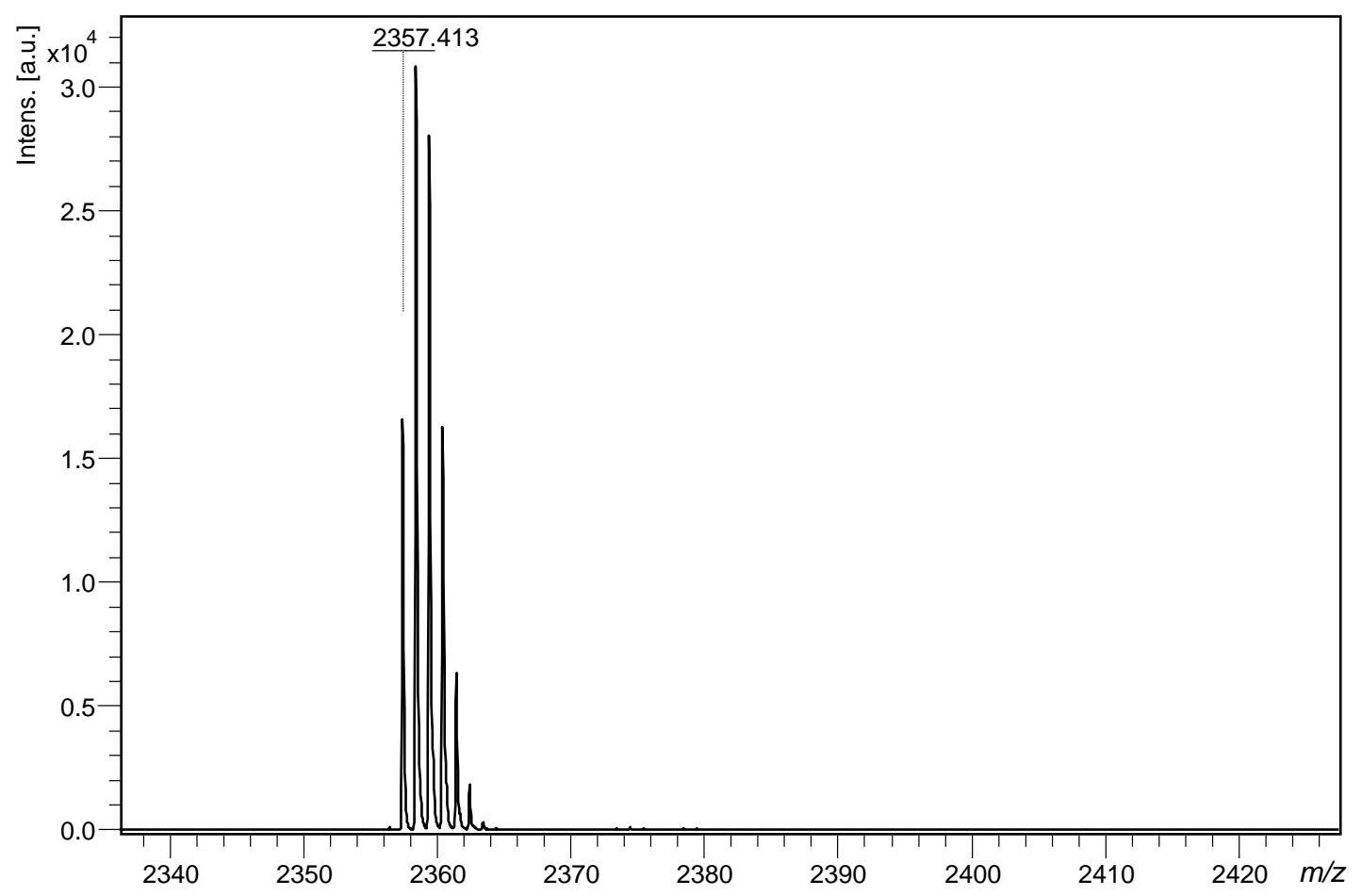

Figure S25. MALDI-TOF mass spectrum of ligand LD. 


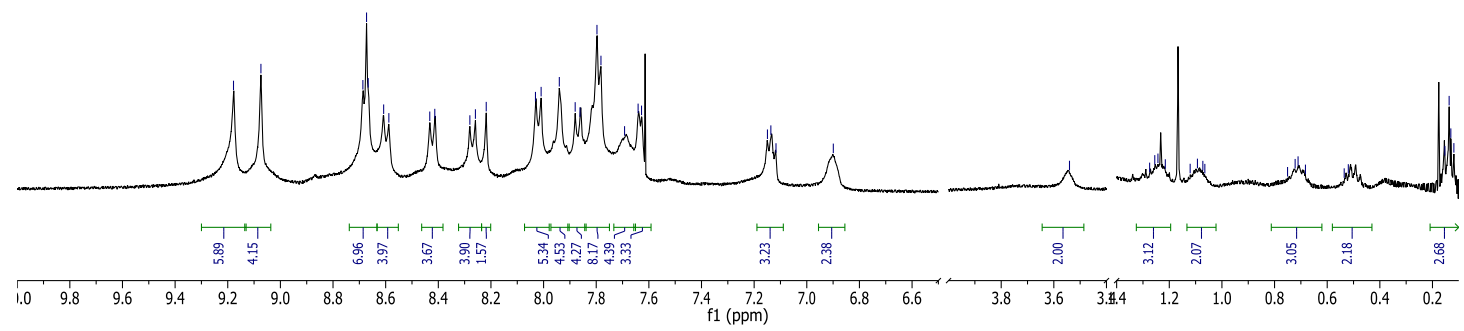

Figure S26. ${ }^{1} \mathrm{H}$ NMR (400 MHz) spectrum of complex $\left[\mathbf{Z n}_{12} \mathbf{L B} \mathbf{6}\right]$.

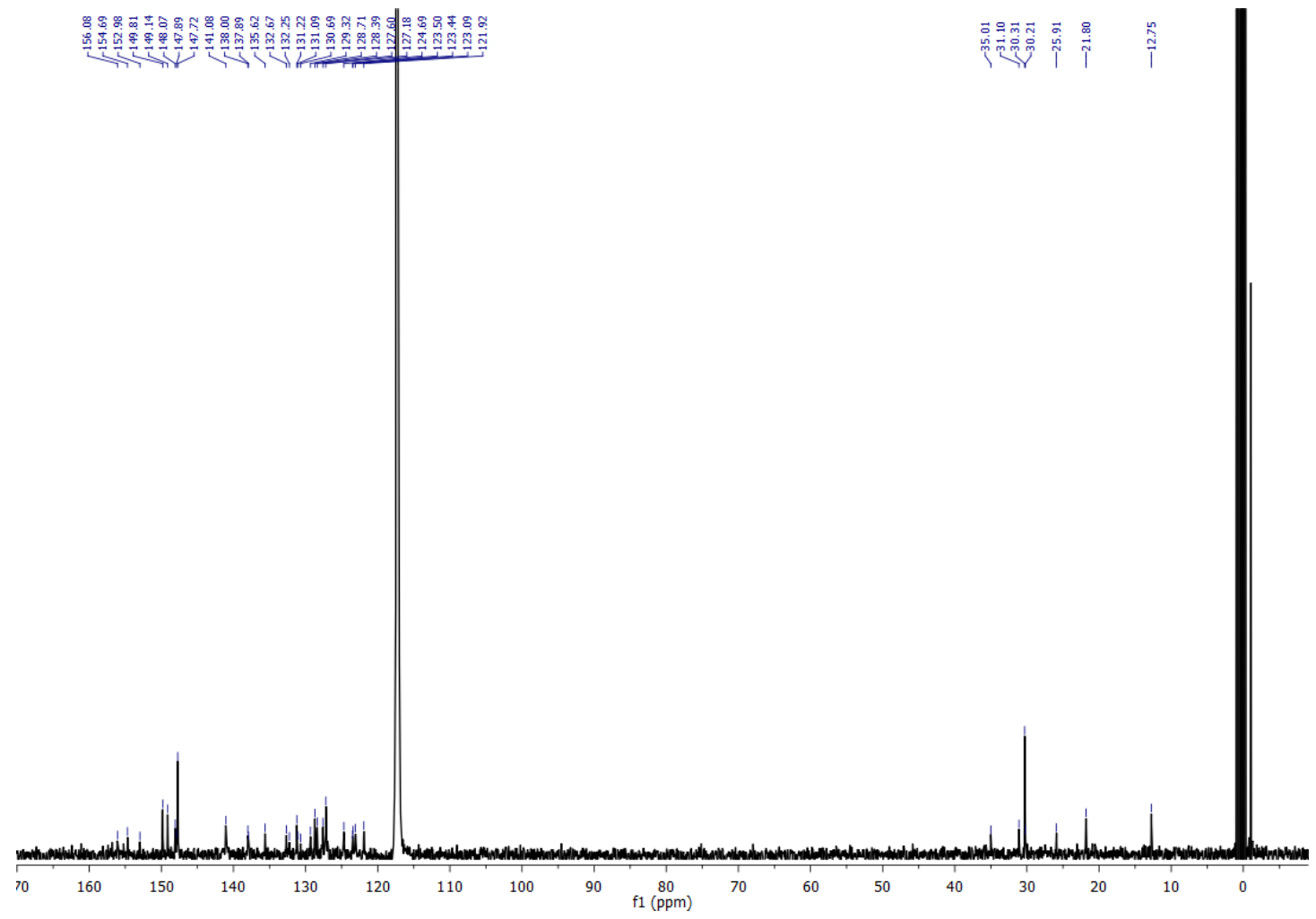

Figure S27. ${ }^{13} \mathrm{C}$ NMR (400 MHz) spectrum of complex $\left[\mathbf{Z n}_{12} \mathbf{L B} 6\right]$. 


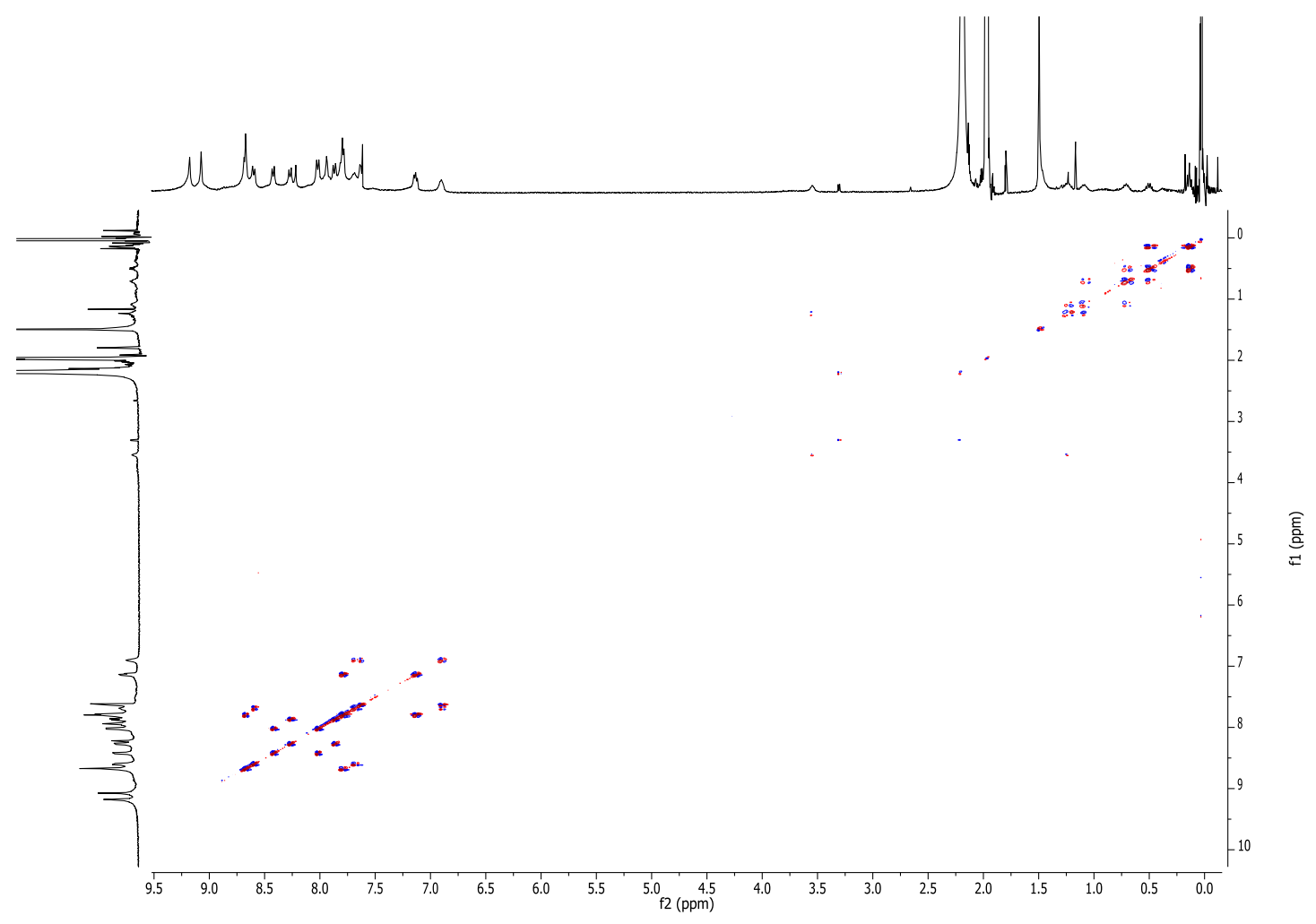

Figure S28. 2D COSY NMR (400 MHz) spectrum of complex [ $\left.\mathbf{Z n}_{\mathbf{1 2}} \mathbf{L} \mathbf{B}_{\mathbf{6}}\right]$.

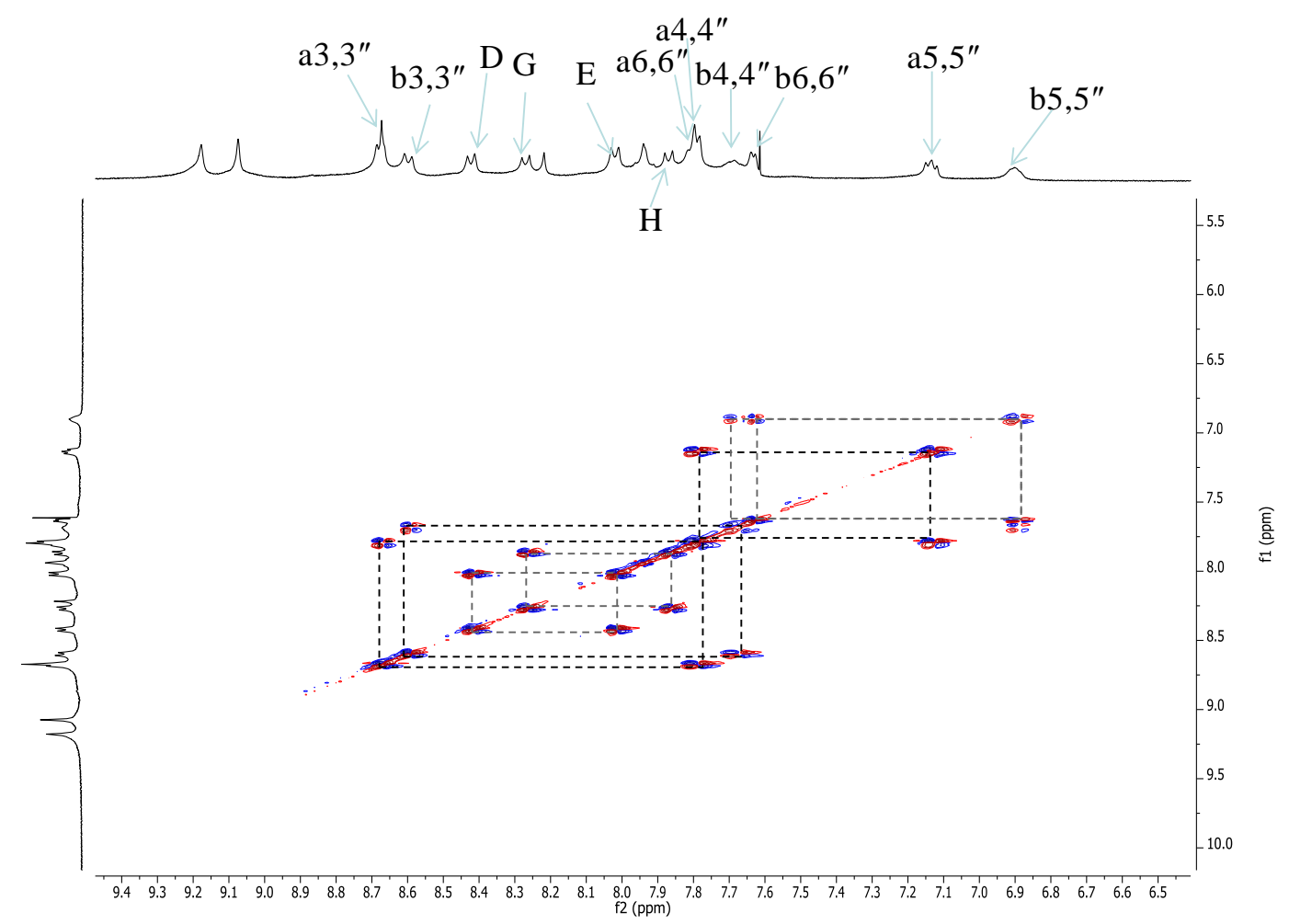

Figure S29. 2D COSY NMR (400 MHz) spectrum of complex [ $\left.\mathbf{Z n}_{\mathbf{1 2}} \mathbf{L B}_{\mathbf{6}}\right]$ (aromatic region). 


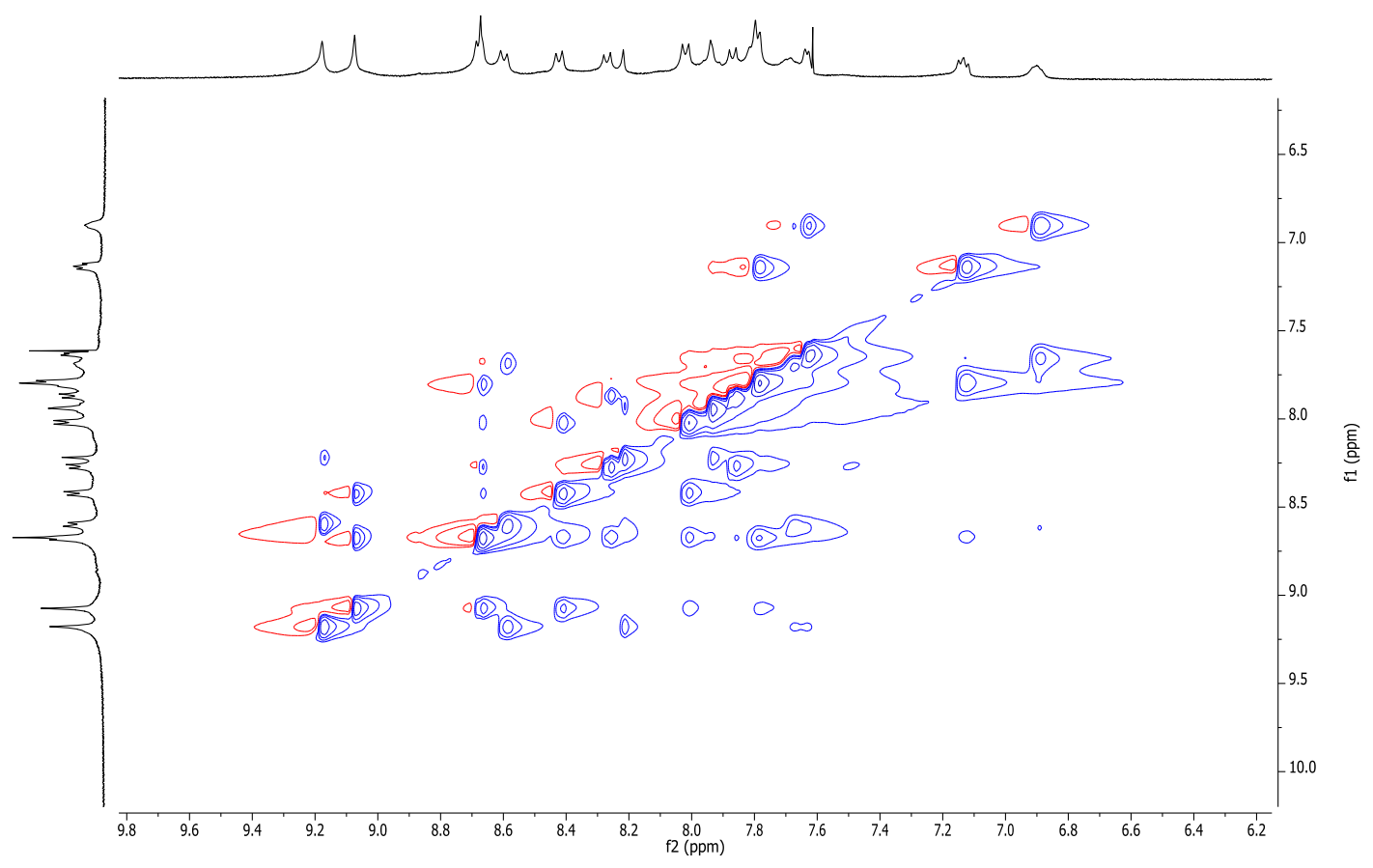

Figure S30. 2D NOESY NMR (400 MHz) spectrum of complex [ $\mathbf{Z n}_{12} \mathbf{L B}_{\mathbf{6}}$ (aromatic region).

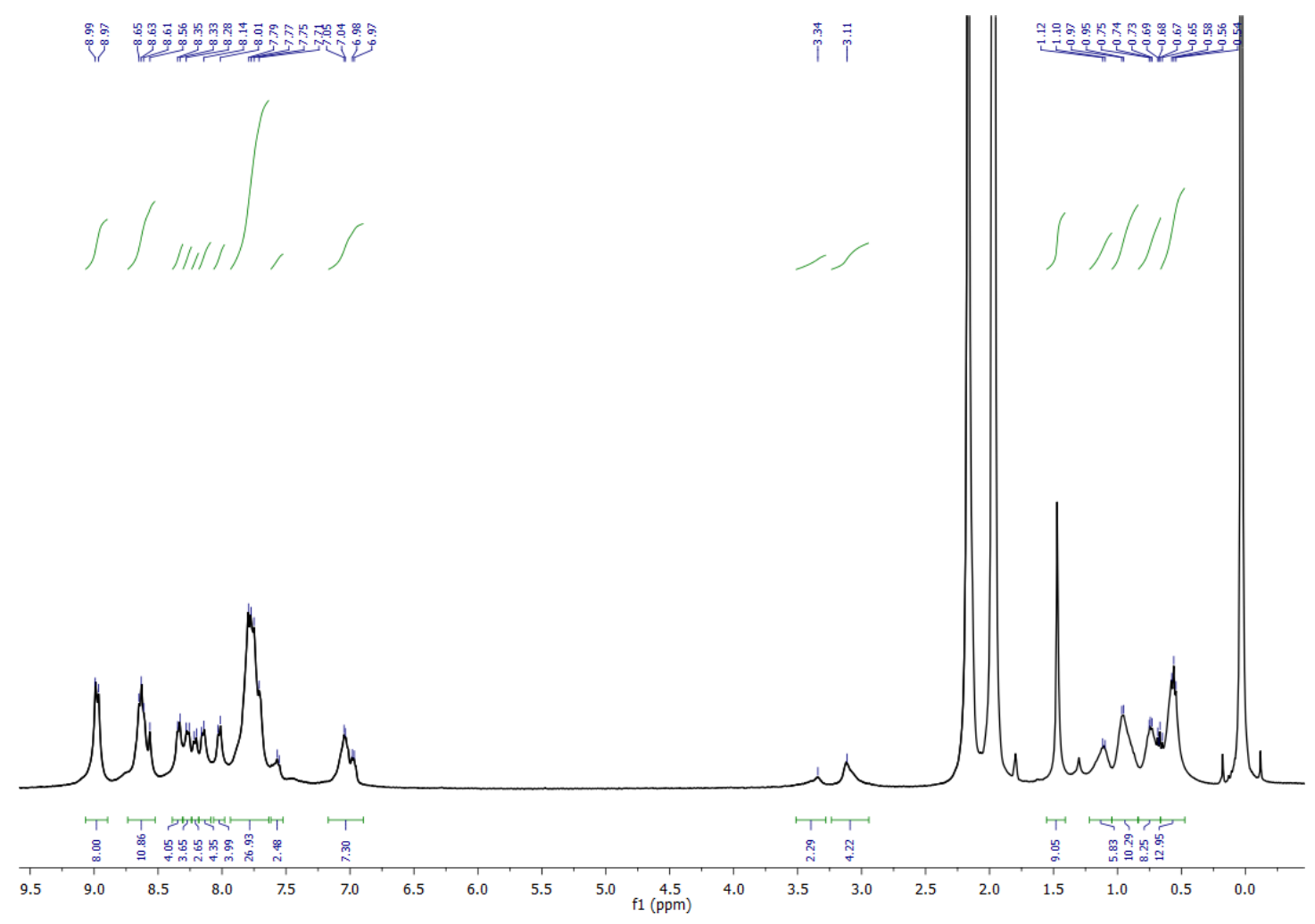

Figure S31. ${ }^{1} \mathrm{H}$ NMR (400 MHz) spectrum of complex [ $\left.\mathbf{Z n}_{\mathbf{1 2}} \mathbf{L} \mathbf{C}_{\mathbf{6}}\right]$. 


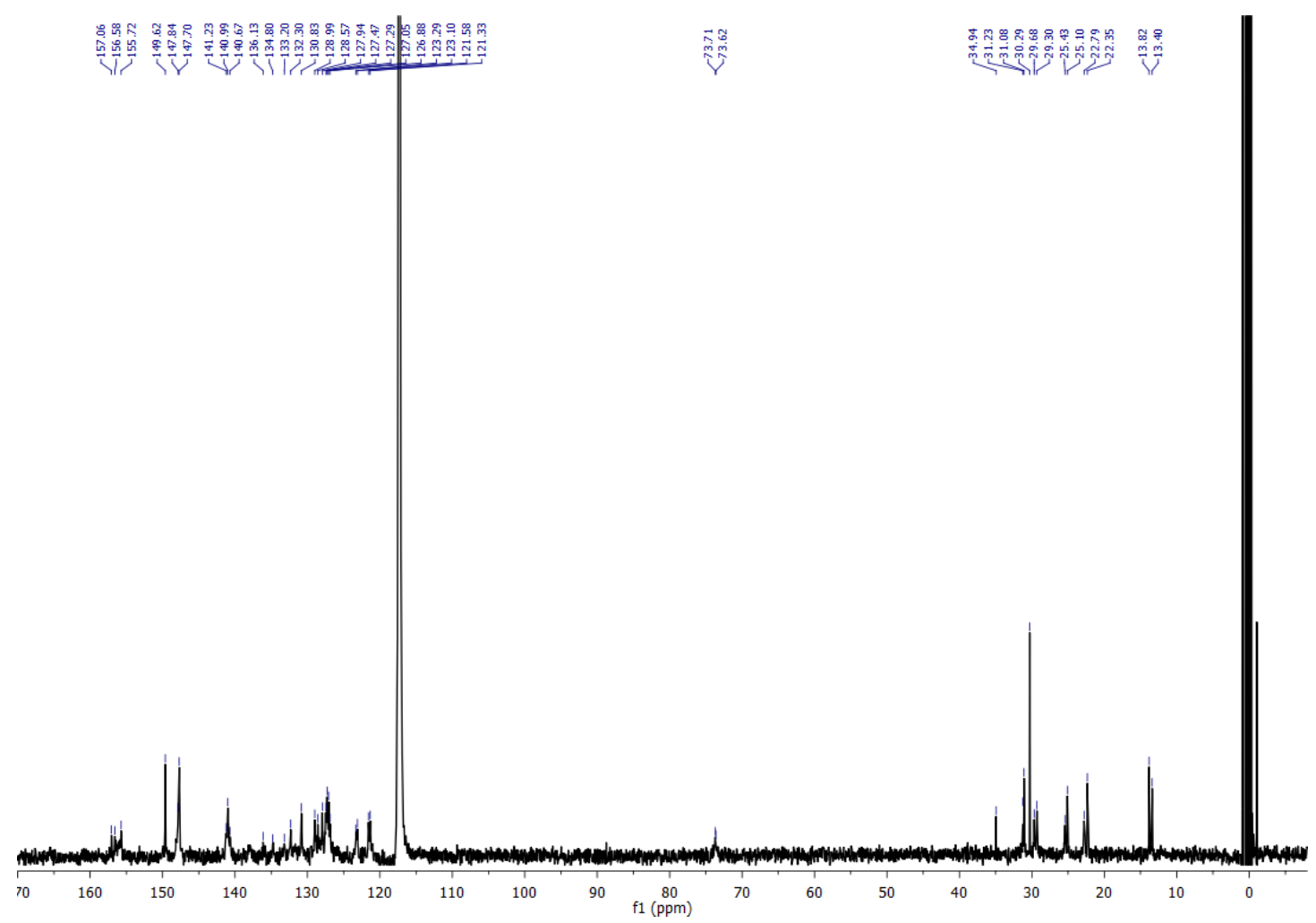

Figure S32. ${ }^{13} \mathrm{C}$ NMR (400 MHz) spectrum of complex $\left[\mathbf{Z n}_{12} \mathbf{L} \mathbf{C}_{\mathbf{6}}\right]$.

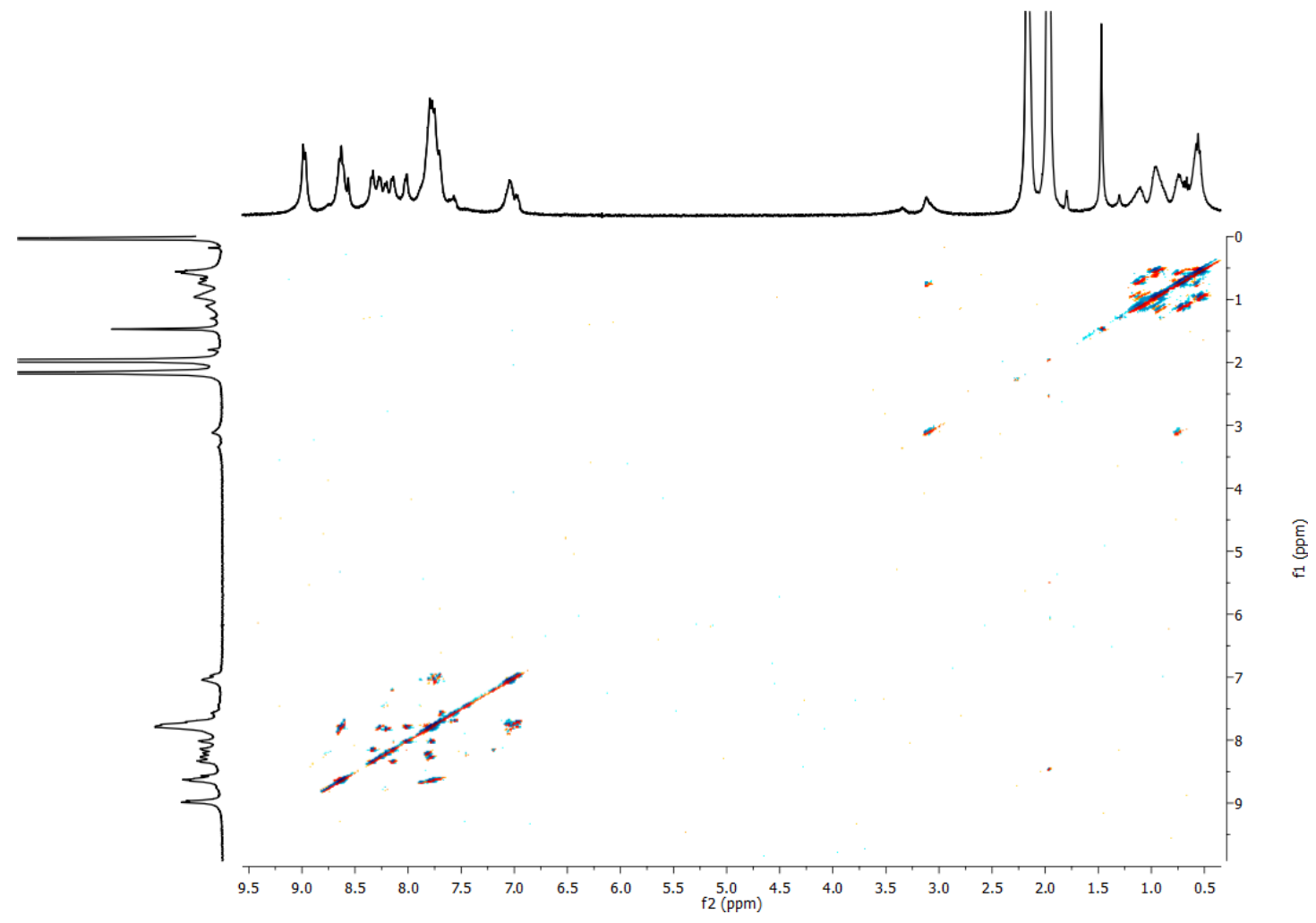

Figure S33. 2D COSY NMR (400 MHz) spectrum of complex [ $\left.\mathbf{Z n}_{\mathbf{1 2}} \mathbf{L} \mathbf{C}_{\mathbf{6}}\right]$. 


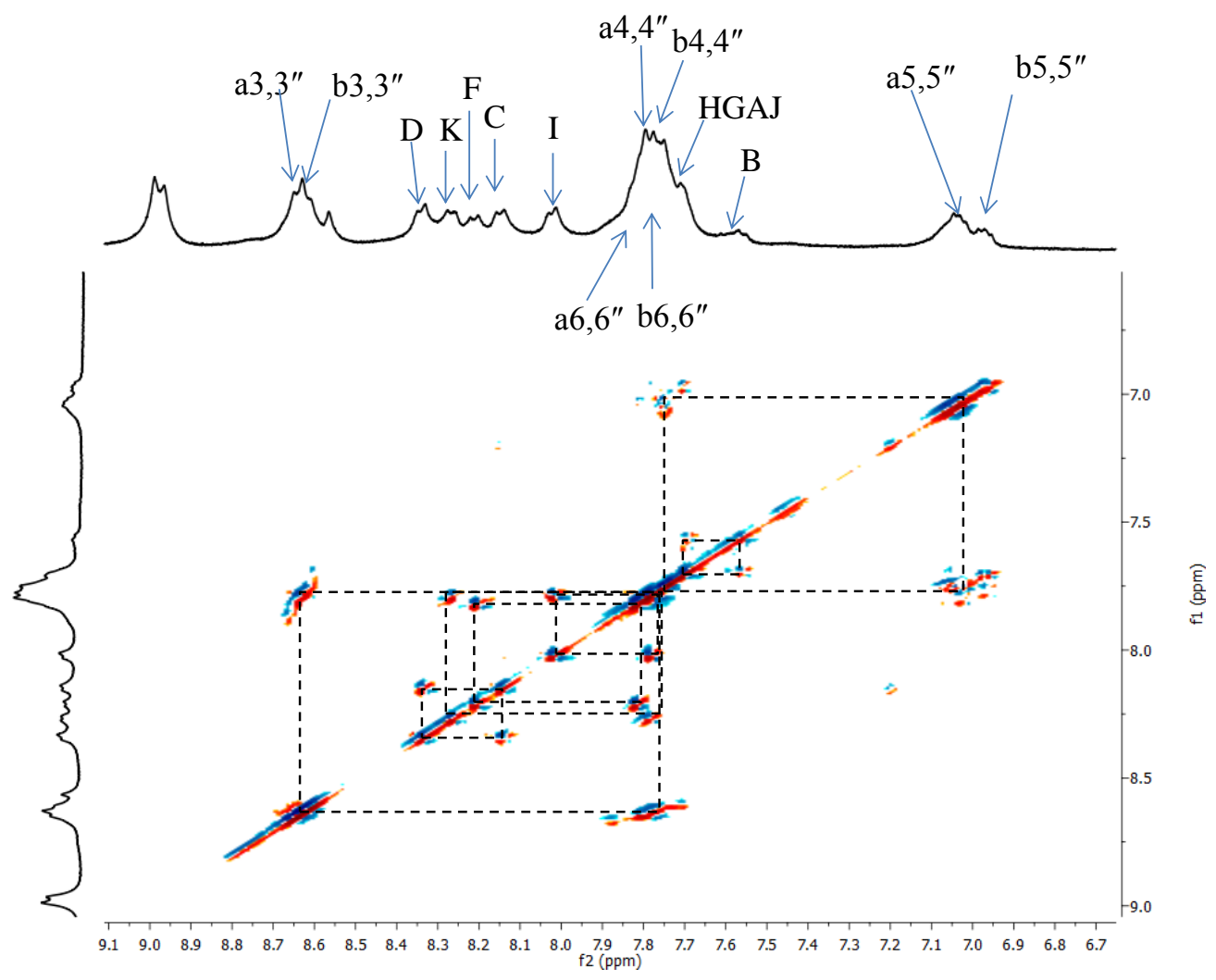

Figure S34. 2D COSY NMR (400 MHz) spectrum of complex [ $\mathbf{Z n}_{\mathbf{1 2}} \mathbf{L} \mathbf{C}_{\mathbf{6}}$ ] (aromatic region).

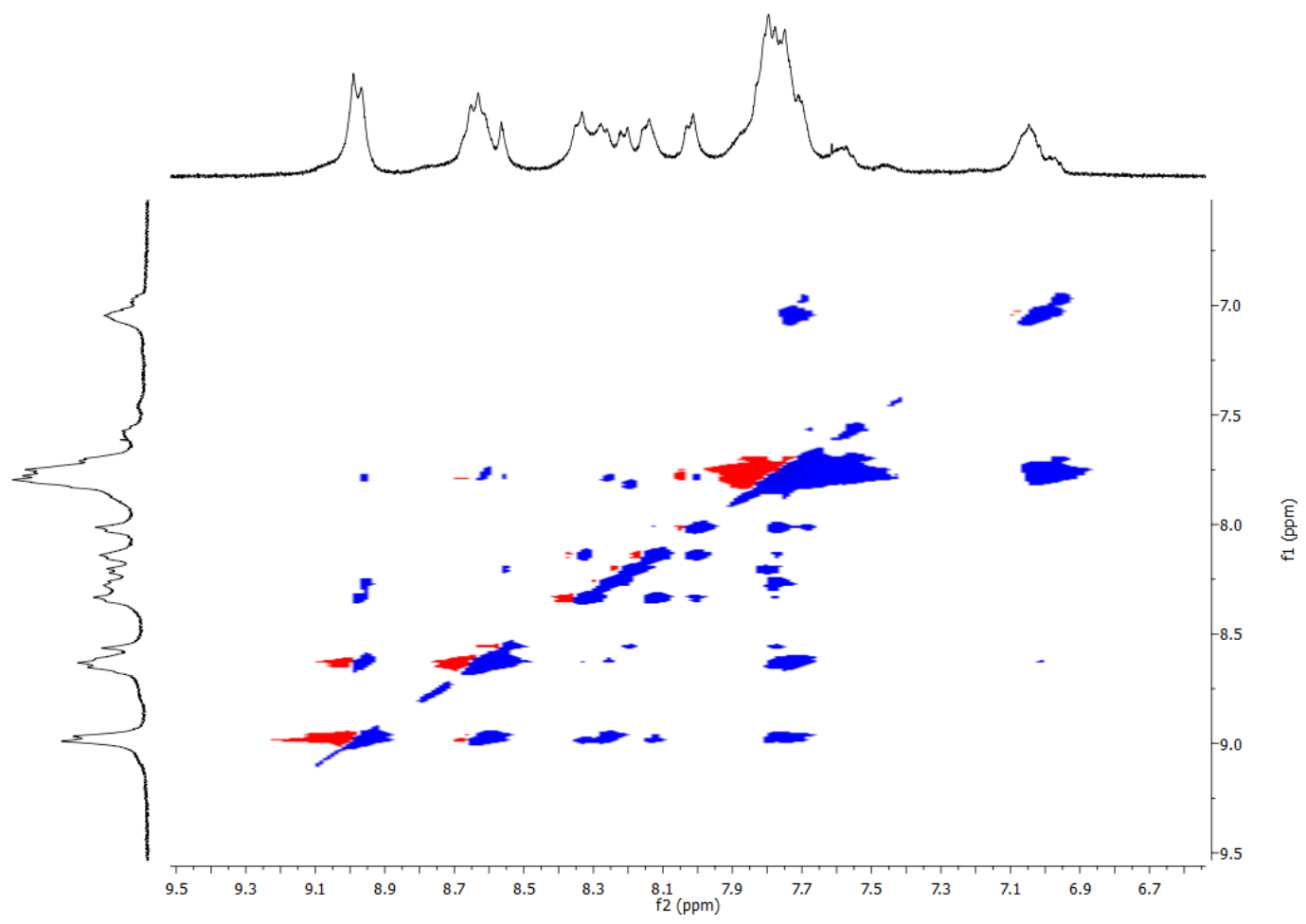

Figure S35. 2D NOESY NMR (400 MHz) spectrum of complex [ $\mathbf{Z n}_{\mathbf{1 2}} \mathbf{L} \mathbf{C}_{\mathbf{6}}$ ] (aromatic 
region).

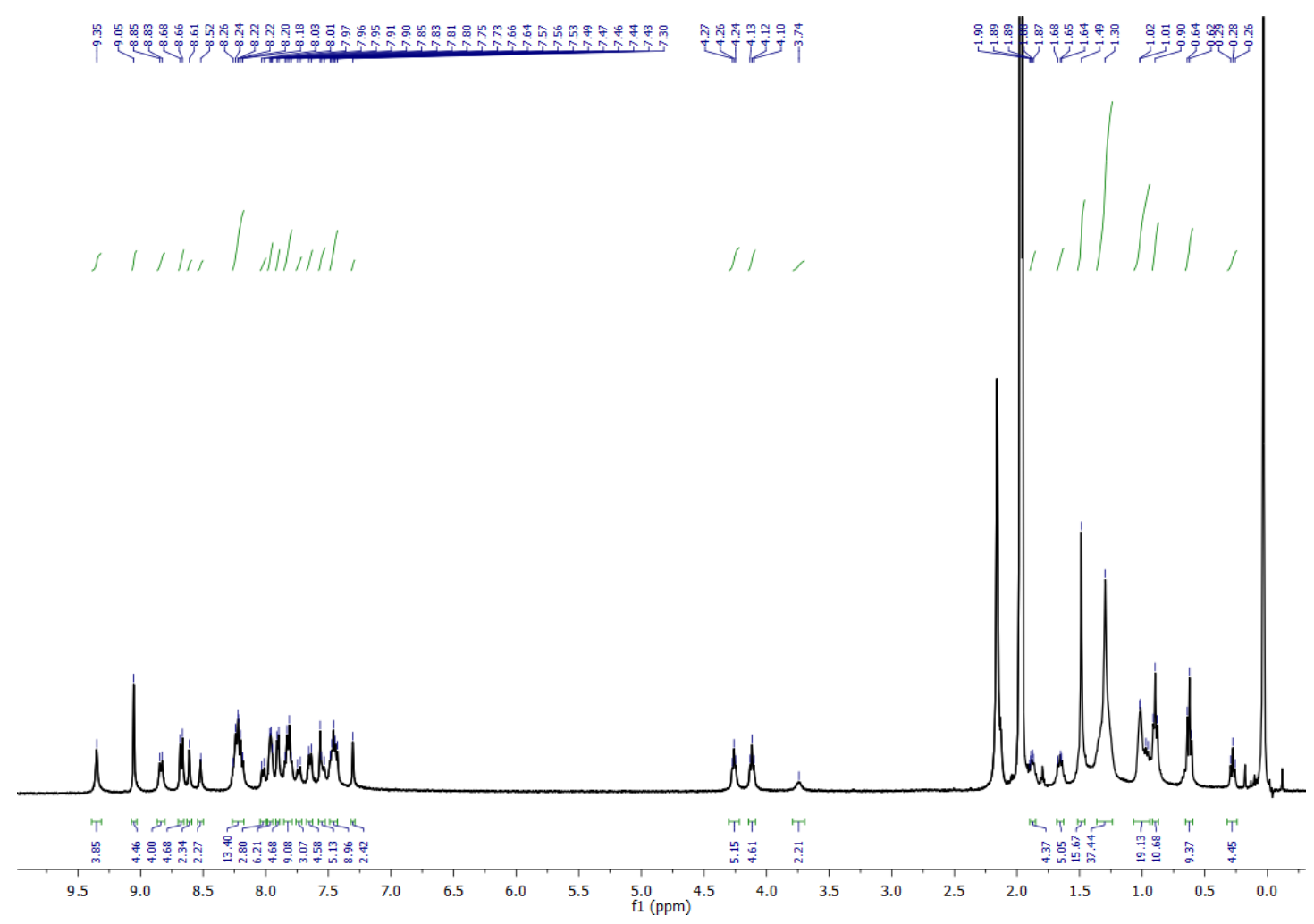

Figure S36. ${ }^{1} \mathrm{H}$ NMR (400 MHz) spectrum of complex $\left[\mathbf{Z n}_{\mathbf{1 2}} \mathbf{L D}_{\mathbf{6}}\right]$.

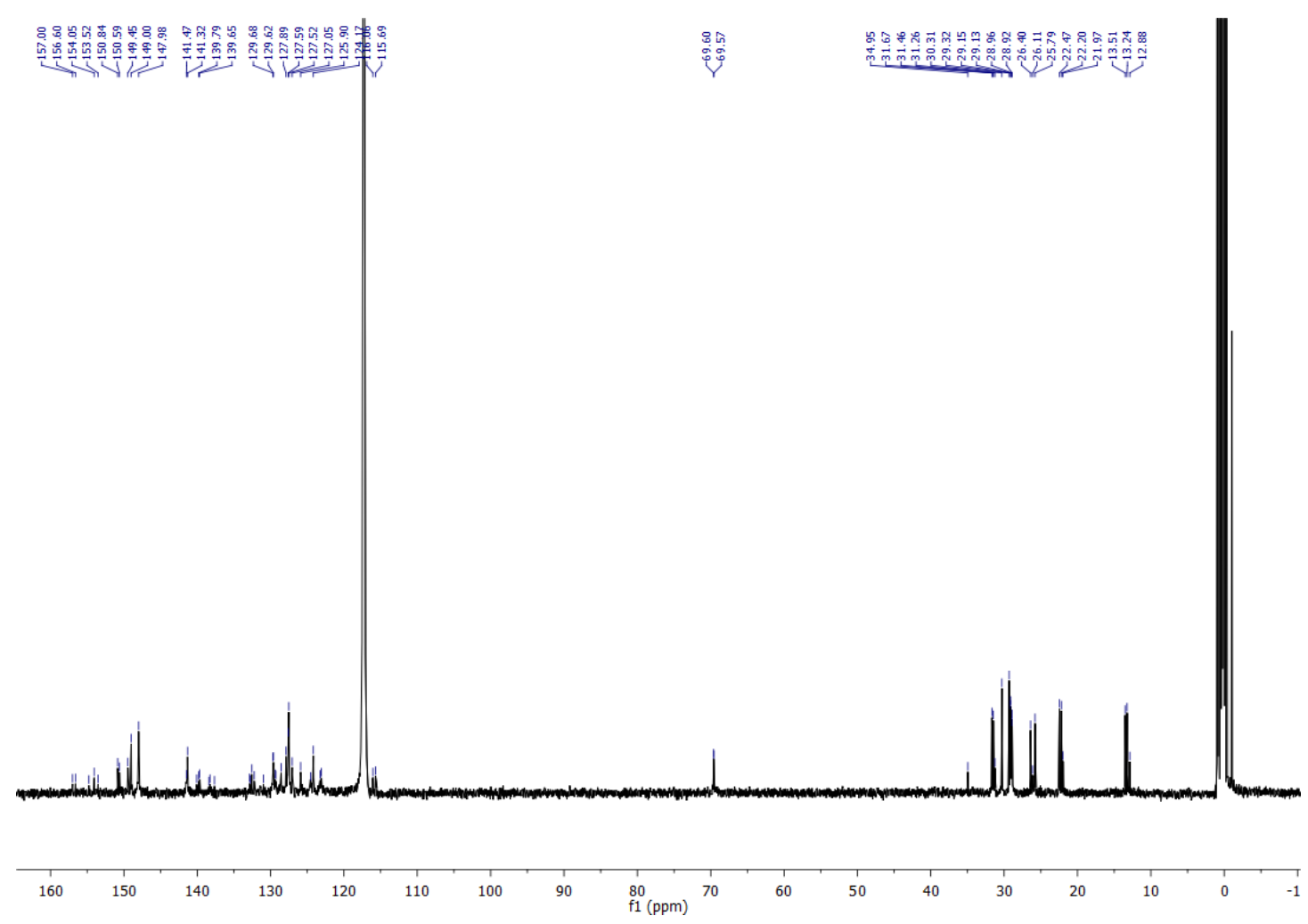

Figure S37. ${ }^{13} \mathrm{C}$ NMR (400 MHz) spectrum of complex $\left[\mathbf{Z n}_{12} \mathbf{L D}_{\mathbf{6}}\right]$. 


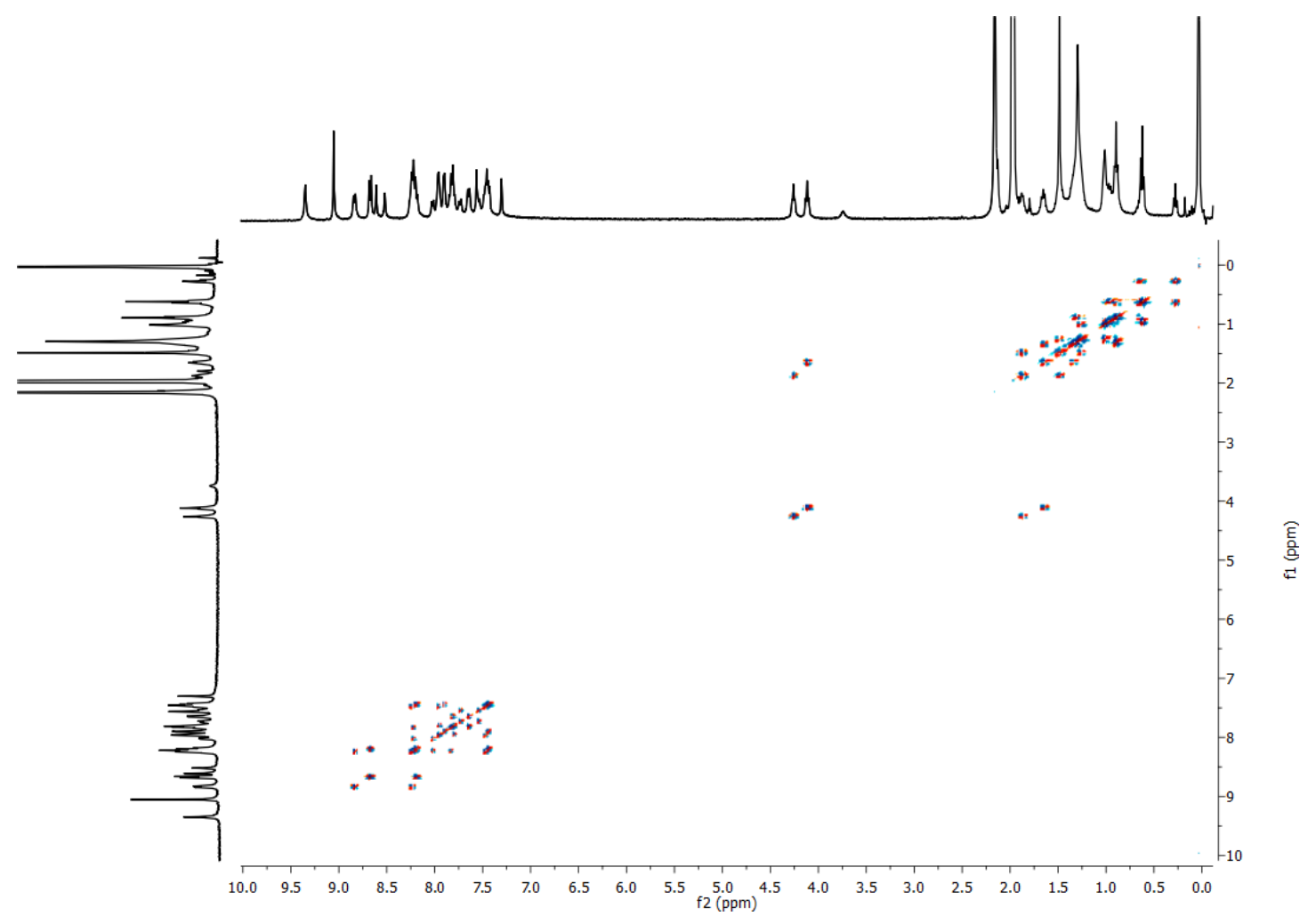

Figure S38. 2D COSY NMR (400 MHz) spectrum of complex [ $\left.\mathbf{Z n}_{12} \mathbf{L} \mathbf{D}_{\mathbf{6}}\right]$.

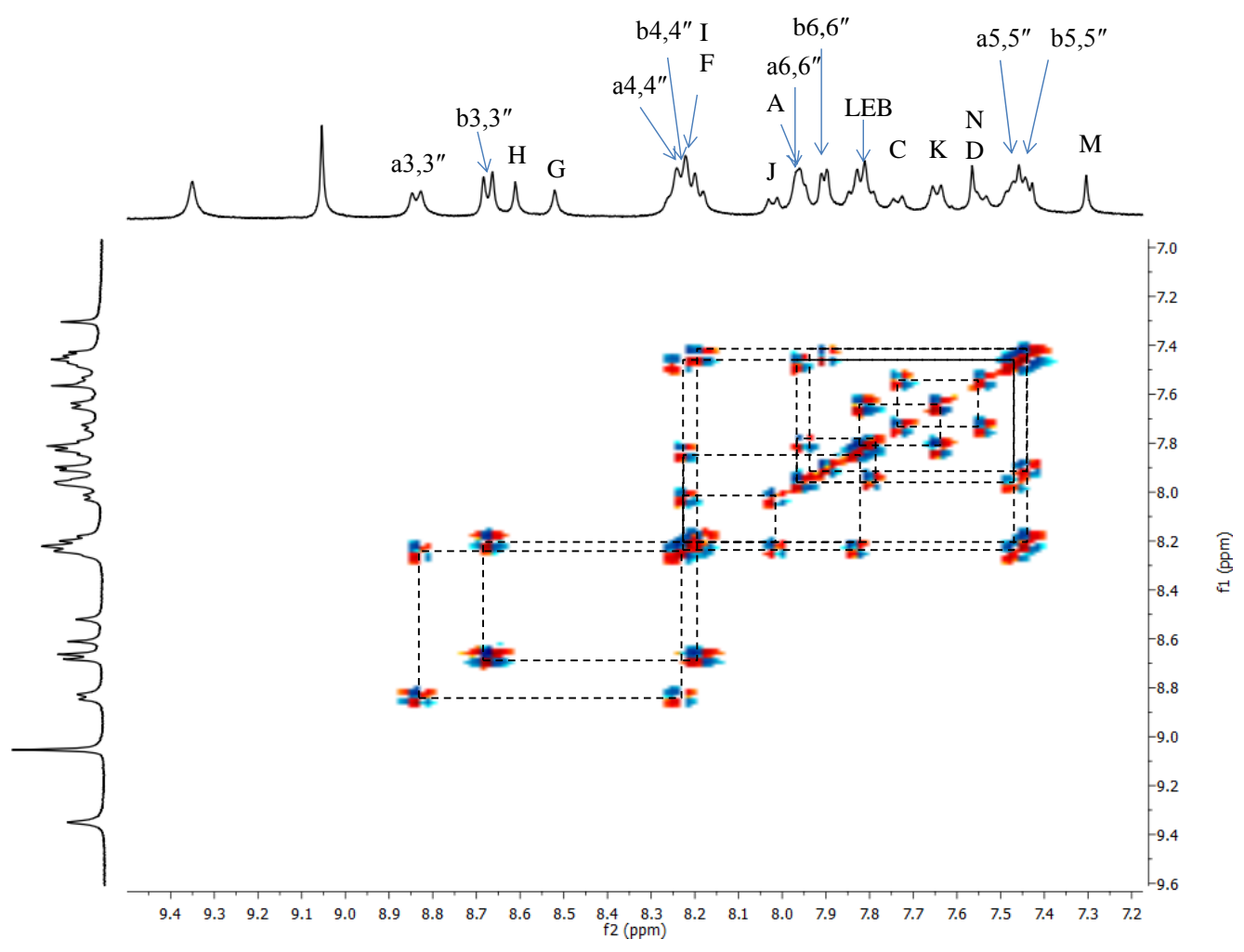

Figure S39. 2D COSY NMR (400 MHz) spectrum of complex [ $\left.\mathbf{Z n}_{\mathbf{1 2}} \mathbf{L} \mathbf{D}_{\mathbf{6}}\right]$ (aromatic region). 


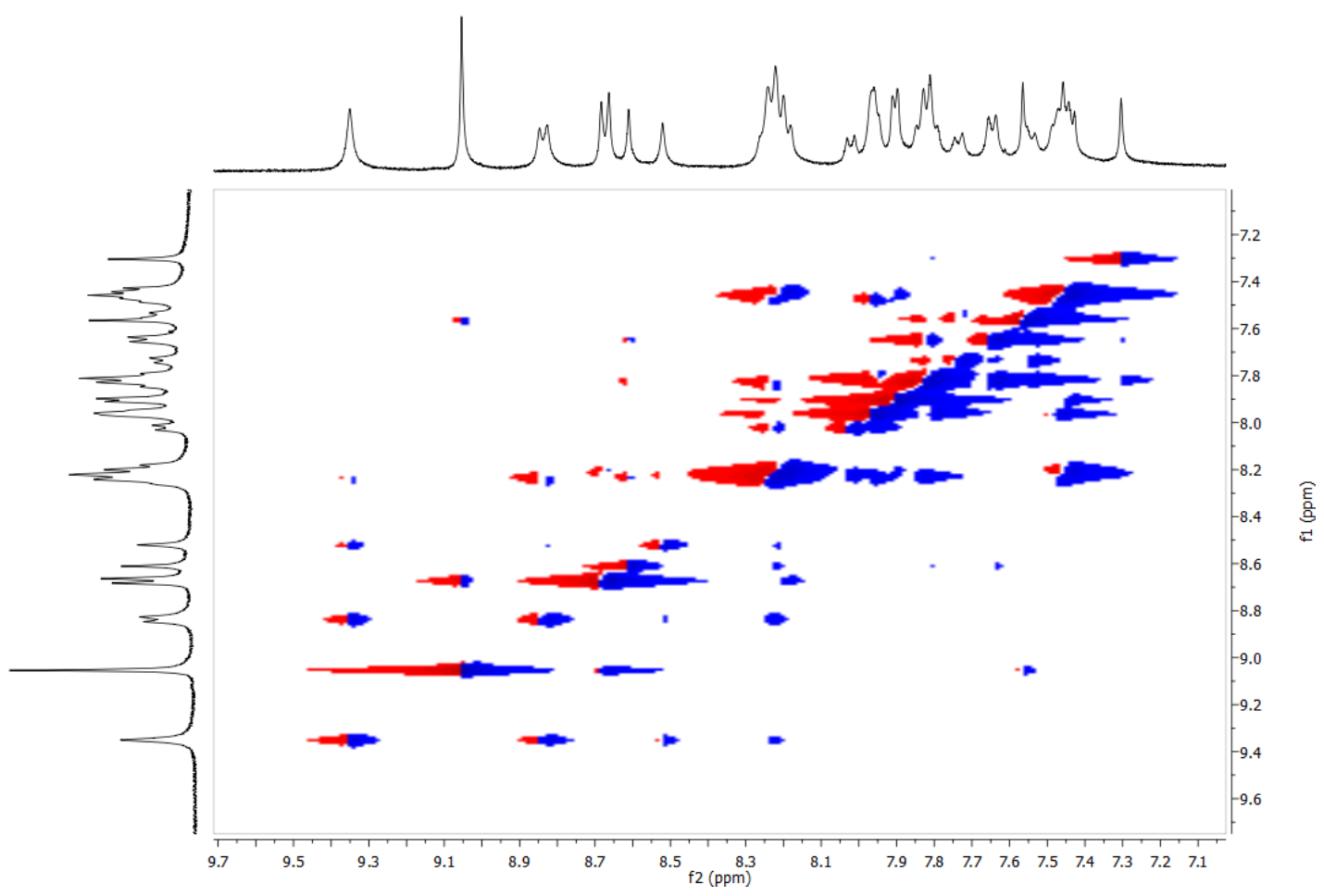

Figure S40. 2D NOESY NMR (400 MHz) spectrum of complex [ $\left.\mathbf{Z n}_{12} \mathbf{L D}_{6}\right]$ (aromatic region). 
9. ESI-MS of multiple macrocycles assembled by ditopic tpy ligand 6 with $\mathrm{Zn}^{2+}$.

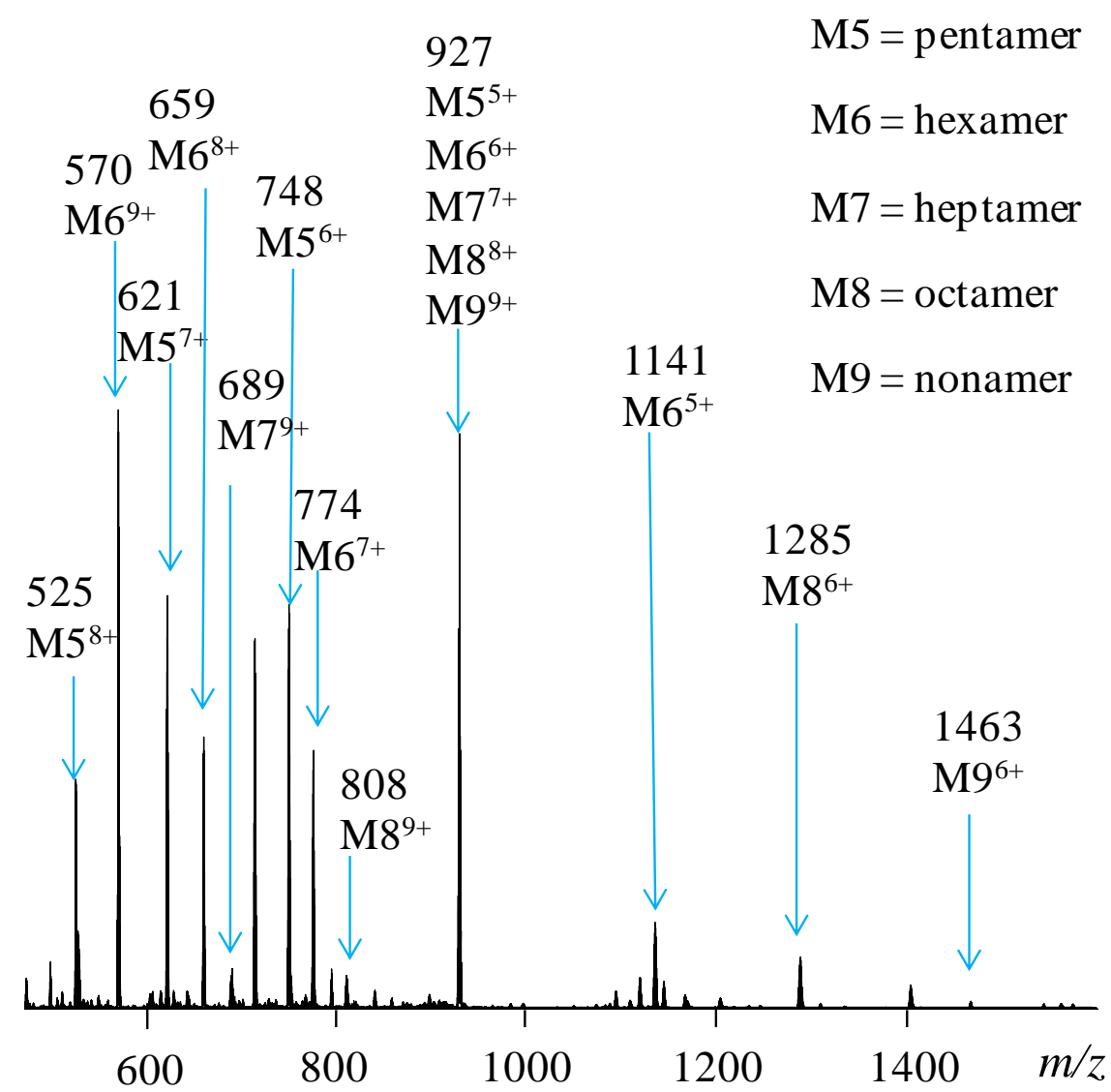

Figure S41. ESI-MS of multiple macrocycles assembled by ditopic tpy ligand 6 with $\mathrm{Zn}^{2+}$. 
10. STM images.
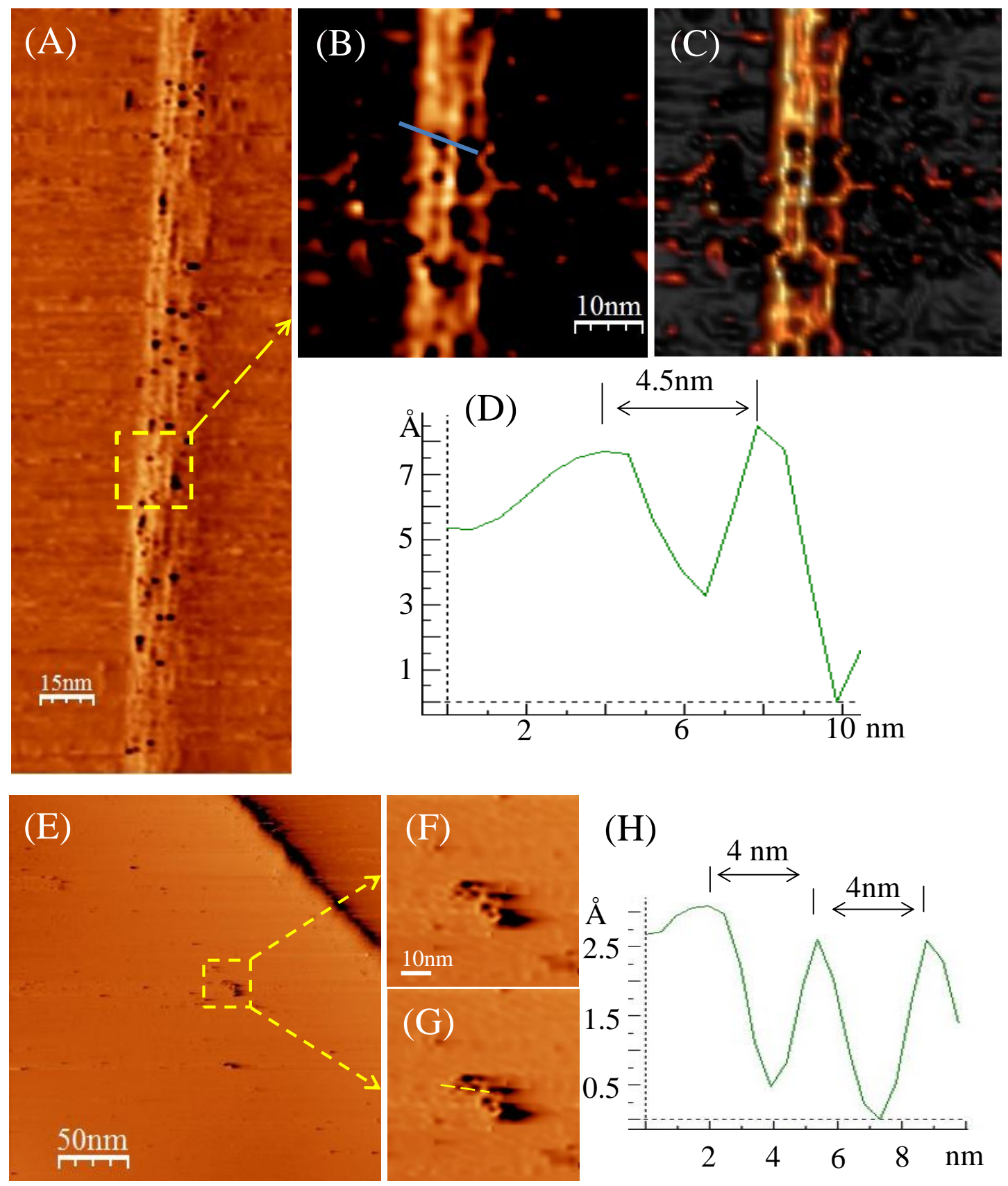

Figure S42. STM images of complex $\left[\mathbf{Z n}_{12} \mathbf{L} \mathbf{C}_{6}\right]$ on HOPG. 

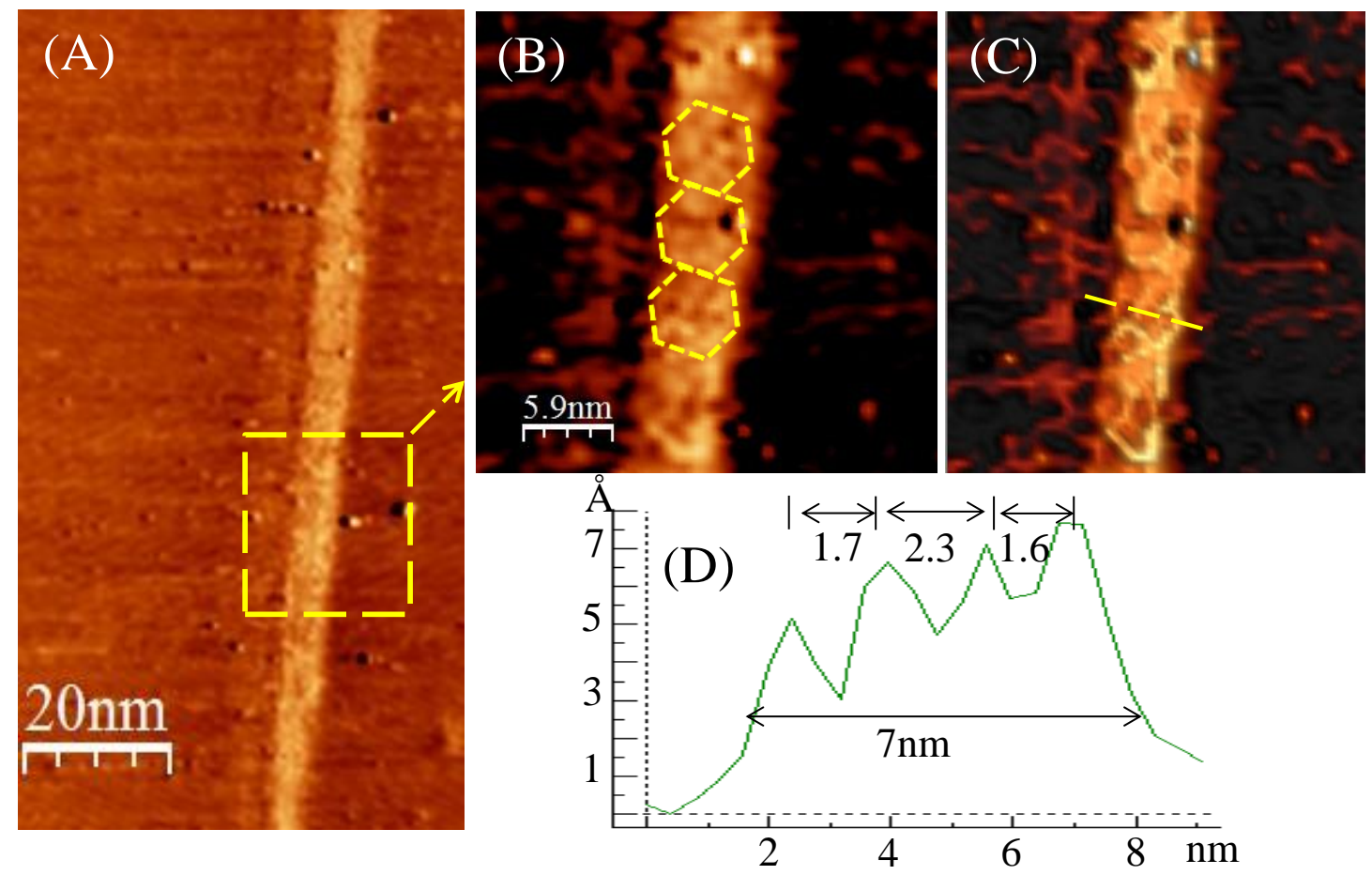

Figure S43. STM images of complex $\left[\mathbf{Z n}_{12} \mathbf{L D}_{6}\right]$ on HOPG. 
11. TEM images.

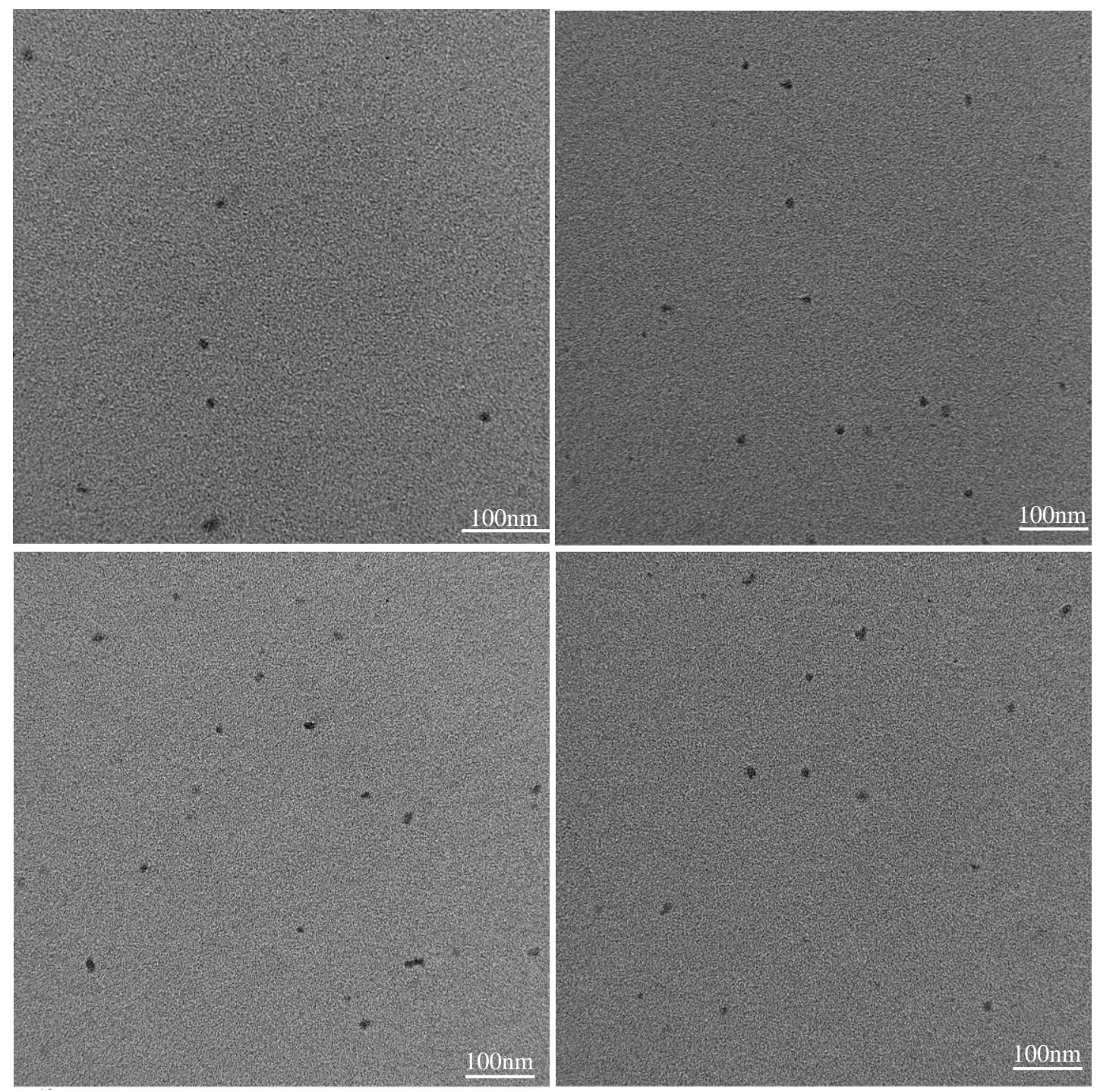

Figure S44. TEM images of complex $\left[\mathbf{Z n}_{12} \mathbf{L B}_{\mathbf{6}}\right]$. 




Figure S45. TEM images of complex $\left[\mathbf{Z n}_{\mathbf{1 2}} \mathbf{L} \mathbf{C}_{\mathbf{6}}\right]$. 


\section{Reference}

(1) Thalassinos, K.; Grabenauer, M.; Slade, S. E.; Hilton, G. R.; Bowers, M. T.; Scrivens, J. H. Anal. Chem. 2008, 81, 248.

(2) http://www.indiana.edu/ clemmer/Research/Cross\%20Section\%20Database/cs_database.php.

(3) Horcas, I.; Fernández, R.; Gómez-Rodríguez, J. M.; Colchero, J.; Gómez-Herrero, J.; Baro, A. M. Rev. Sci. Instrum. 2007, 78, 013705.

(4) Katagiri, S.; Sakamoto, R.; Maeda, H.; Nishimori, Y.; Kurita, T.; Nishihara, H. Chem. Eur. J. 2013, 19, 5088 .

(5) Drewry, J. A.; Duodu, E.; Mazouchi, A.; Spagnuolo, P.; Burger, S.; Gradinaru, C. C.; Ayers, P.; Schimmer, A. D.; Gunning, P. T. Inorg. Chem. 2012, 51, 8284.

(6) Lei, S.; Ver Heyen, A.; De Feyter, S.; Surin, M.; Lazzaroni, R.; Rosenfeldt, S.; Ballauff, M.; Lindner, P.; Mössinger, D.; Höger, S. Chem. Eur. J. 2009, 15, 2518.

(7) Sharghi, H.; Nasseri, M. A.; Niknam, K. J. Org. Chem. 2001, 66, 7287.

(8) Zhu, X.; Bheemireddy, S. R.; Sambasivarao, S. V.; Rose, P. W.; Torres Guzman, R.; Waltner, A. G.; DuBay, K. H.; Plunkett, K. N. Macromolecules 2016, 49, 127.

(9) Dance, Z. E. X.; Ahrens, M. J.; Vega, A. M.; Ricks, A. B.; McCamant, D. W.; Ratner, M. A.; Wasielewski, M. R. J. Am. Chem. Soc. 2008, 130, 830. 